Swarthmore College

Works

\title{
KELT-1b: A Strongly Irradiated, Highly Inflated, Short Period, 27 Jupiter-Mass Companion Transiting A Mid-F Star
}

\author{
R. J. Siverd \\ T. G. Beatty \\ J. Pepper \\ J. D. Eastman \\ K. Collins
}

See next page for additional authors

Follow this and additional works at: https://works.swarthmore.edu/fac-physics

Part of the Astrophysics and Astronomy Commons

Let us know how access to these works benefits you

\section{Recommended Citation}

R. J. Siverd, T. G. Beatty, J. Pepper, J. D. Eastman, K. Collins, A. Bieryla, D. W. Latham, L. A. Buchhave, Eric L.N. Jensen, J. R. Crepp, R. Street, K. G. Stassun, B. S. Gaudi, P. Berlind, M. L. Calkins, D. L. DePoy, G. A. Esquerdo, B. J. Fulton, G. Fürész, J. C. Geary, A. Gould, L. Hebb, J. F. Kielkopf, J. L. Marshall, R. Pogge, K. Z. Stanek, R. P. Stefanik, A. H. Szentgyorgyi, M. Trueblood, P. Trueblood, A. M. Stutz, and J. L. Van Saders. (2012). "KELT-1b: A Strongly Irradiated, Highly Inflated, Short Period, 27 Jupiter-Mass Companion Transiting A Mid-F Star". Astrophysical Journal. Volume 761, Issue 2. DOI: 10.1088/0004-637X/761/2/123 https://works.swarthmore.edu/fac-physics/49

This work is brought to you for free by Swarthmore College Libraries' Works. It has been accepted for inclusion in Physics \& Astronomy Faculty Works by an authorized administrator of Works. For more information, please contact myworks@swarthmore.edu. 


\section{Authors}

R. J. Siverd, T. G. Beatty, J. Pepper, J. D. Eastman, K. Collins, A. Bieryla, D. W. Latham, L. A. Buchhave, Eric L.N. Jensen, J. R. Crepp, R. Street, K. G. Stassun, B. S. Gaudi, P. Berlind, M. L. Calkins, D. L. DePoy, G. A.

Esquerdo, B. J. Fulton, G. Fürész, J. C. Geary, A. Gould, L. Hebb, J. F. Kielkopf, J. L. Marshall, R. Pogge, K.

Z. Stanek, R. P. Stefanik, A. H. Szentgyorgyi, M. Trueblood, P. Trueblood, A. M. Stutz, and J. L. Van Saders 


\title{
KELT-1b: A STRONGLY IRRADIATED, HIGHLY INFLATED, SHORT PERIOD, 27 JUPITER-MASS COMPANION TRANSITING A MID-F STAR
}

\author{
Robert J. Siverd ${ }^{1}$, Thomas G. Beatty ${ }^{2}$, Joshua Pepper ${ }^{1}$, Jason D. Eastman ${ }^{3,4}$, Karen Collins $^{5}$, Allyson Bieryla ${ }^{6}$, \\ David W. Latham ${ }^{6}$, Lars A. Buchhave ${ }^{7,8}$, Eric L. N. Jensen ${ }^{9}$, Justin R. Crepp ${ }^{10,11}$, Rachel Street ${ }^{3}$, \\ Keivan G. Stassun ${ }^{1,12}$, B. Scott Gaudi ${ }^{2}$, Perry Berlind ${ }^{6}$, Michael L. Calkins ${ }^{6}$, D. L. DePoy ${ }^{13,14}$, Gilbert A. Esquerdo ${ }^{6}$, \\ Benjamin J. Fulton ${ }^{3}, 15$, Gábor Fứrész ${ }^{6}$, John C. Geary ${ }^{6}$, Andrew Gould ${ }^{2}$, Leslie Hebb ${ }^{1}$, John F. KielkopF ${ }^{5}$, \\ Jennifer L. Marshall ${ }^{13}$, Richard Pogge ${ }^{2}$, K. Z. StaneK ${ }^{2}$, Robert P. StefaniK ${ }^{6}$, Andrew H. Szentgyorgyi ${ }^{6}$, \\ Mark Trueblood ${ }^{16}$, Patricia Trueblood ${ }^{16}$, Amelia M. Stutz ${ }^{17,18}$, and Jennifer L. van Saders ${ }^{2}$ \\ ${ }^{1}$ Department of Physics and Astronomy, Vanderbilt University, Nashville, TN 37235, USA \\ ${ }^{2}$ Department of Astronomy, The Ohio State University, 140 W. 18th Avenue, Columbus, OH 43210, USA \\ ${ }^{3}$ Las Cumbres Observatory Global Telescope Network, 6740 Cortona Drive, Suite 102, Santa Barbara, CA 93117, USA \\ ${ }^{4}$ Department of Physics Broida Hall, University of California, Santa Barbara, CA 93106, USA \\ ${ }^{5}$ Department of Physics \& Astronomy, University of Louisville, Louisville, KY 40292, USA \\ ${ }^{6}$ Harvard-Smithsonian Center for Astrophysics, 60 Garden Street, Cambridge, MA 02138, USA \\ ${ }^{7}$ Niels Bohr Institute, University of Copenhagen, Juliane Maries vej 30, DK-21S00 Copenhagen, Denmark \\ ${ }^{8}$ Centre for Star and Planet Formation, Geological Museum, Øster Voldgade 5, DK-1350 Copenhagen, Denmark \\ ${ }^{9}$ Department of Physics and Astronomy, Swarthmore College, Swarthmore, PA 19081, USA \\ ${ }^{10}$ Department of Astrophysics, California Institute of Technology, Pasadena, CA 91125, USA \\ ${ }^{11}$ Department of Physics, University of Notre Dame, 225 Nieuwland Science Hall, Notre Dame, IN 46556, USA \\ ${ }^{12}$ Department of Physics, Fisk University, Nashville, TN 37208, USA \\ ${ }^{13}$ Department of Physics \& Astronomy, Texas A\&M University, College Station, TX 77843, USA \\ ${ }^{14}$ George P. and Cynthia Woods Mitchell Institute for Fundamental Physics and Astronomy, Texas A\&M University, College Station, TX 77843, USA \\ ${ }^{15}$ Institute for Astronomy, University of Hawaii, 2680 Woodlawn Drive, Honolulu, HI 96822, USA \\ ${ }^{16}$ Winer Observatory, Sonoita, AZ 85637, USA \\ ${ }^{17}$ Max Planck Institute for Astronomy, Heidelberg, Germany \\ ${ }^{18}$ Department of Astronomy \& Steward Observatory, University of Arizona, Tucson, AZ 85721, USA \\ Received 2012 June 6; accepted 2012 October 26; published 2012 December 4
}

\begin{abstract}
We present the discovery of KELT-1b, the first transiting low-mass companion from the wide-field Kilodegree Extremely Little Telescope-North (KELT-North) transit survey. A joint analysis of the spectroscopic, radial velocity, and photometric data indicates that the $V=10.7$ primary is a mildly evolved mid-F star with $T_{\text {eff }}=6516 \pm 49 \mathrm{~K}, \log g=4.228_{-0.021}^{+0.014}$, and $[\mathrm{Fe} / \mathrm{H}]=0.052 \pm 0.079$, with an inferred mass $M_{*}=1.335 \pm 0.063 M_{\odot}$ and radius $R_{*}=1.471_{-0.035}^{+0.045} R_{\odot}$. The companion is a low-mass brown dwarf or a super-massive planet with mass $M_{P}=27.38 \pm 0.93 M_{\mathrm{Jup}}$ and radius $R_{P}=1.116_{-0.029}^{+0.038} R_{\mathrm{Jup}}$. The companion is on a very short $(\sim 29 \mathrm{hr})$ period circular orbit, with an ephemeris $T_{c}\left(\mathrm{BJD}_{\mathrm{TDB}}\right)=2455909.29280 \pm 0.00023$ and $P=1.217501 \pm 0.000018$ days. KELT-1b receives a large amount of stellar insolation, resulting in an estimated equilibrium temperature assuming zero albedo and perfect redistribution of $T_{\mathrm{eq}}=2423_{-27}^{+34} \mathrm{~K}$. Comparison with standard evolutionary models suggests that the radius of KELT- $1 \mathrm{~b}$ is likely to be significantly inflated. Adaptive optics imaging reveals a candidate stellar companion to KELT-1 with a separation of $588 \pm 1$ mas, which is consistent with an M dwarf if it is at the same distance as the primary. Rossiter-McLaughlin measurements during transit imply a projected spin-orbit alignment angle $\lambda=2 \pm 16 \mathrm{deg}$, consistent with a zero obliquity for KELT-1. Finally, the $v \sin I_{*}=56 \pm 2 \mathrm{~km} \mathrm{~s}$ of the primary is consistent at $\sim 2 \sigma$ with tidal synchronization. Given the extreme parameters of the KELT- 1 system, we expect it to provide an important testbed for theories of the emplacement and evolution of short-period companions, as well as theories of tidal dissipation and irradiated brown dwarf atmospheres.
\end{abstract}

Key words: planetary systems - stars: individual: KELT-1, TYC 2785-2130-1 - techniques: photometric techniques: spectroscopic

Online-only material: color figures, machine-readable tables

\section{INTRODUCTION}

The most information-rich exoplanetary systems are those in which the companion happens to transit in front of its parent star. Transiting systems are enormously useful for enabling detailed measurements of a seemingly endless array of physical properties of extrasolar planets and their host stars (see reviews by Winn 2009, 2010). The most basic properties that can be measured using transiting planets are the planet mass and radius, and so average density. These parameters alone allow for interesting constraints on the internal composition and structure of planets (Guillot 2005; Fortney et al. 2007; Rogers \& Seager 2010; Miller \& Fortney 2011). In addition to these basic parameters, transiting planets enable the study of their atmospheres (Seager \& Sasselov 2000; Charbonneau et al. 2002; Vidal-Madjar et al. 2003; Seager \& Deming 2010) and thermal emission (Deming et al. 2005; Charbonneau et al. 2005; Knutson et al. 2008). They also allow measurement of planetary and stellar oblateness, rotation rate, and spin-orbit alignment (Seager \& Hui 2002; Spiegel et al. 2007; Carter \& Winn 2010; Rossiter 1924; McLaughlin 1924; Winn et al. 2005; Gaudi \& Winn 2006). Transiting planets may also be searched 
for associated rings and moons (Brown et al. 2001; Barnes \& Fortney 2004; Tusnski \& Valio 2011). Further, variations in transit timing may indicate the presence of other bodies in the system (Holman \& Murray 2005; Agol et al. 2005; Steffen \& Agol 2005; Ford \& Gaudi 2006; Ford \& Holman 2007; Kipping 2009). With sufficiently precise observations, one may constrain the presence of planets with masses smaller than that of the Earth (Agol \& Steffen 2006; Carter \& Winn 2010).

The high scientific value of transiting planet systems motivated the first dedicated wide-field transit surveys, which by now have identified over 100 transiting systems (TrES; Alonso et al. 2004; XO, McCullough et al. 2006; HATNet, Bakos et al. 2007; SuperWASP, Collier Cameron et al. 2007a, QES, Alsubai et al. 2011). Although there is substantial diversity in their design, strategy, and sensitivity, these surveys can be grossly characterized as having relatively small cameras with apertures of the order of $10 \mathrm{~cm}$, and detectors with relatively wide fields of view of tens of square degrees. These surveys are primarily sensitive to giant, close-in planets with radii $R_{P} \gtrsim 0.5 R_{\mathrm{Jup}}$ and periods of $P \lesssim 10$ days, orbiting relatively bright FGK stars with $V \sim 10-12$.

The space-based missions CoRoT (Baglin 2003) and Kepler (Borucki et al. 2010) have dramatically expanded the parameter space of transit surveys, enabling the detection of planets with sizes down to that of the Earth and below, planets with periods of several years, and planets orbiting a much broader range of host stars. Furthermore, their large target samples have allowed the detection of rare and therefore interesting planetary systems. These missions have already announced nearly 100 confirmed planets, and the Kepler mission has announced an additional 2300 candidates (Batalha et al. 2012), most of which are smaller than Neptune. Notable individual discoveries include the first detection of a transiting super-Earth (Léger et al. 2009), the detection of a "temperate" gas giant with a relatively long period of $\sim 100$ days (Deeg et al. 2010), the first multi-planet transiting systems (Steffen et al. 2010; Holman et al. 2010; Latham et al. 2011; Lissauer et al. 2011), the first circumbinary planets (Doyle et al. 2011; Welsh et al. 2012), and the detection of planets with radius of $\lesssim R_{\oplus}$ (Muirhead et al. 2012; Fressin et al. 2012).

Although Kepler and CoRoT have revolutionized our understanding of the demographics of planets, the opportunities for follow-up of the systems detected by these missions are limited. By design, both missions primarily monitor relatively faint stars with $V \gtrsim 12$. Consequently, many of the follow-up observations discussed above that are generically enabled by transiting systems are not feasible for the systems detected by Kepler and CoRoT. Detailed characterization of the majority of these systems will therefore be difficult or impossible. There is thus an ongoing need to discover transiting planets orbiting the bright stars, as well as to increase the diversity of such systems.

All else being equal, the brightest stars hosting transiting planets are the most valuable. Larger photon flux permits more instruments and/or facilities to be employed for follow-up, allows subtler effects to be probed, reduces statistical uncertainties, and generally allows for improved or more extensive calibration procedures that help to control systematic errors. Furthermore, brighter stars are also easier to characterize and are more likely to have pre-existing information, such as proper motions, parallaxes, metallicities, effective temperatures, angular diameters, and broadband colors.

The majority of the brightest $(V \lesssim 8$ ) FGK dwarfs in the sky have been monitored using precision radial velocity (RV) surveys for many years, and as a result most of the giant planets with periods of less than a few years orbiting these stars have already been discovered (e.g., Wright et al. 2012). A smaller subset of these stars have been monitored over a shorter time baseline with the sensitivity needed to detect Neptuneand super-Earth-mass planets. Because of the low a priori transit probability for all but short period planets, the transiting systems constitute a very small fraction of this sample. To date, seven planets first discovered via RV have subsequently been discovered to also transit; all of the host stars for these planets are brighter than $V=9$. Although there are projects that aim to increase this sample (Kane et al. 2009), the overall yield is expected to be small.

Because RV surveys generically require spectroscopic observations that are observationally expensive and must be obtained in series, it is more efficient to discover transiting planets around the much more abundant fainter stars by first searching for the photometric transit signal, and then following these up with targeted RV observations to eliminate false positives and measure the planet mass. However, in order to compensate for the rarity and low duty cycle, many stars must be monitored over a long time baseline. Photometric transit surveys that target brighter stars therefore require larger fields of view. Most of the original transit surveys had fields of view and exposure times that were optimized to detect planets orbiting stars with $V \gtrsim 10$. Indeed, only $\sim 20$ transiting planets orbiting stars with $V \leqslant 10$ are currently known $(\sim 40$ with $V \lesssim 11)$. Of those with $V \leqslant 10$, $\sim 40 \%$ were originally detected by RV surveys.

The Kilodegree Extremely Little Telescope-North (KELTNorth) transit survey (Pepper et al. 2007) was designed to detect giant, short-period transiting planets orbiting the brightest stars that are not readily accessible to RV surveys. Pepper et al. (2003) determined the optimal hardware setup specifically to detect transiting planets orbiting stars with $V \sim 8-10$, and based on the specified design requirements in that paper, the KELT-North survey telescope system was constructed using offthe-shelf, high-end consumer equipment. In fact, as the current detection demonstrates, KELT has exceeded its design goals and is sensitive to transiting systems in some favorable cases down to $V \sim 12$.

In addition to the goal of filling in the magnitude gap between RV and other transit surveys, the KELT-North survey also has the potential to detect fainter systems with $V \gtrsim 10$ that are in the magnitude range of previous surveys, but were missed or overlooked for various reasons. The detection discussed in this paper is an example of this opportunity. Here, the fact that the KELT-North survey is only now starting to vet candidates, more than eight years after the first candidates were announced by other transit surveys, can be seen as an advantage. In particular, previous surveys have established the existence of massive brown dwarf (BD) companions (Deleuil et al. 2008; Irwin et al. 2010; Bouchy et al. 2011b; Johnson et al. 2011; Bouchy et al. 2011a) and have demonstrated the feasibility of detecting low-mass companions to hot, rapidly rotating stars (Collier Cameron et al. 2010). Partially in response to these results, the KELT-North survey deliberately broadened our search targets to include hot and/or rapidly rotating stars, which were previously neglected by many transit surveys. The evolving perception of what kinds of stars constitute viable transit search targets played an interesting role in the discovery of KELT-1b, as discussed in Section 3.2.

The KELT-North survey has been collecting data since 2006 September and has acquired a sufficient number of high-quality 
Table 1

KELT-North BLS Candidate Selection Criteria

\begin{tabular}{lccc}
\hline \hline Signal detection efficiency & SDE $>7.0$ & Depth & $\delta<0.05$ \\
Signal to pink-noise & SPN $>7.0$ & $\chi^{2}$ ratio & $\frac{\Delta \chi^{2}}{\Delta \chi_{-}^{2}}>1.5$ \\
Fraction from one night & $f_{1 n}<0.8$ & Duty cycle & $q<0.1$ \\
\hline
\end{tabular}

images to detect transit candidates. We have been systematically identifying and vetting transit candidates since 2011 February, and in this paper we report our first confirmed low-mass transiting companion, which we designate KELT-1b. KELT-1b has a mass of $\sim 27 M_{\mathrm{Jup}}$, and we will therefore follow convention and refer to it as a "brown dwarf" throughout the majority of this paper. However, as we discuss in Section 6.1, we are, in fact, agnostic about its true nature and therefore how it should be categorized.

The outline of this paper is as follows. In order to introduce the survey and provide the appropriate context for our discovery, in Section 2 we summarize the properties of the KELT-North survey and our procedure for candidate selection. In Section 3, we review the observations of KELT-1, starting with the properties of the candidate in the KELT-North data, and then summarize the follow-up photometry, spectroscopy, and high-contrast imaging. Section 5 describes our analysis and characterization of the host star and its substellar companion. In Section 6, we provide a speculative discussion of the possible implications of this unique system for theories of the emplacement and tidal evolution of short-period substellar companions, models of the structure and atmosphere of BDs, and the demographics of substellar companions to stars. We briefly summarize in Section 7.

For quick reference, Table 1 lists the values of the light curve statistics used to select KELT candidates, Table 2 provides a summary of the observations of the KELT- 1 system, Table 3 lists various collected properties and measurements of the KELT-1 host star, Table 4 lists median values and $68 \%$ confidence intervals for the physical and orbital parameters of the KELT-1 system, Table 5 lists median values and $68 \%$ confidence intervals for the light curve and radial velocity parameters of the KELT-1 system, Table 6 lists the inferred transit times, Tables 7 and 8 list the radial velocity and bisector measurements, and Tables 9-17 list the photometric measurements.

\section{THE KELT-NORTH SURVEY}

Because this is the first paper from the KELT-North survey, we describe the survey, selection criteria, and follow-up observations and reduction methodology in some detail. Readers who are not interested in these details, but are rather primarily interested in the properties and implications of the KELT-1b system, can skip to Section 5.

\subsection{KELT-North Instrumentation and Survey Strategy}

The KELT-North survey instrument consists of a collection of commercially available equipment, chosen to meet the requirements of Pepper et al. (2003) and tuned to find the few brightest stars with transiting giant planets in the Northern sky. The optical system consists of an Apogee AP16E $(4 \mathrm{~K} \times 4 \mathrm{~K}$ $9 \mu \mathrm{m}$ pixels) thermo-electrically cooled CCD camera attached using a custom mounting plate to a Mamiya camera lens with an $80 \mathrm{~mm}$ focal length and $42 \mathrm{~mm}$ aperture $(f / 1.9)$. The resultant field of view of the detector is $26^{\circ} \times 26^{\circ}$ at roughly $23^{\prime \prime}$ pixel $^{-1}$, allowing simultaneous observation of nearly 40,000 stars in typical high Galactic latitude fields. The medium-format image size is markedly larger than the CCD detector (which measures $37 \times 37 \mathrm{~mm}$ ) which greatly reduces the severity of vignetting across the large field of view. At the same time, the small aperture permits longer exposures, which improve observing efficiency (assuming fixed camera readout time). A Kodak Wratten \#8 red-pass filter is mounted in front of the lens to further reduce the impact of atmospheric reddening (which primarily affects blue wavelengths) on our photometry. The resultant bandpass resembles a widened Johnson-Cousins $R$ band. This optical

Table 2

Summary of Observations

\begin{tabular}{|c|c|c|c|c|}
\hline Observatory & UT Dates & No. of Obs & Wavelength & Section; Table \\
\hline PvdKO & 2011 Dec 3-2011 Dec 4 & 151 & $i$ & Section 3.4 .1 ; T9 \\
\hline $\mathrm{HAO}$ & 2011 Dec 10 & 266 & $i$ & Section $3.4 .3 ; \mathrm{T} 11$ \\
\hline FLWO & 2011 Dec 16 & 362 & $z$ & Section 3.4 .4 ; T12 \\
\hline \multicolumn{5}{|c|}{ Photometry, Secondary Eclipse } \\
\hline ULMO & 2011 Dec 2 & 115 & $i$ & Section 3.4 .2 \\
\hline FTN/LCOGT & 2011 Dec 30 & 72 & PS- $Z^{\mathrm{a}}$ & Section 3.4 .5 \\
\hline ULMO & 2012 Jan 4 & 121 & $i$ & Section 3.4 .2 \\
\hline FLWO/TRES & UT 2012 Jan 7 & 42 & $3900-8900 \AA$ & Section 3.3 ; T8 \\
\hline \multicolumn{5}{|c|}{ Adaptive Optics Imaging } \\
\hline Keck/NIRC2 & UT 2012 Jan 7 & & $H, K^{\prime}$ & Section 3.5 \\
\hline
\end{tabular}

Notes. ULMO: University of Louisville Moore Observatory; PvdKO: Peter van de Kamp Observatory; HAO: Hereford Arizona Observatory; FLWO: Fred Lawrence Whipple Observatory; FTN: Faulkes Telescope North; LCOGT: Las Cumbres Observatory Global Telescope Network.

a Pan-STARRS-Z 
Table 3

KELT-1 Stellar Properties

\begin{tabular}{|c|c|c|c|c|}
\hline Parameter & Description (Units) & Value & Source & Ref. \\
\hline Names & & $\begin{array}{c}\text { TYC 2785-2130-1 } \\
\text { SC 02785-02130 } \\
\text { 2MASS J00012691+3923017 }\end{array}$ & & \\
\hline$\alpha_{\mathrm{J} 2000}$ & & 00:01:26.92 & Tycho & 1 \\
\hline$\delta_{\mathrm{J} 2000}$ & & $+39: 23: 01.7$ & Tycho & 1 \\
\hline$B_{T}$ & & $11.363 \pm 0.065$ & Tycho-2 & 2 \\
\hline$V_{T}$ & & $10.701 \pm 0.057$ & Tycho-2 & 2 \\
\hline$J$ & & $9.682 \pm 0.022$ & 2MASS & 3 \\
\hline$H$ & & $9.534 \pm 0.030$ & 2MASS & 3 \\
\hline K & & $9.437 \pm 0.019$ & 2MASS & 3 \\
\hline WISE1 & & $12.077 \pm 0.022$ & WISE & 4 \\
\hline WISE2 & & $12.732 \pm 0.020$ & WISE & 4 \\
\hline WISE3 & & $14.655 \pm 0.030$ & WISE & 4 \\
\hline$\mu_{\alpha}$ & Proper motion in R.A. $\left(\right.$ mas $\left.\mathrm{yr}^{-1}\right) \ldots$ & $-10.1 \pm 0.7$ & NOMAD & 5 \\
\hline$\mu_{\delta}$ & Proper motion in decl. $\left(\right.$ mas $\left.\mathrm{yr}^{-1}\right) \ldots$ & $-9.4 \pm 0.7$ & NOMAD & 5 \\
\hline$\gamma_{\mathrm{abs}}$ & Absolute systemic $\mathrm{RV}\left(\mathrm{km} \mathrm{s}^{-1}\right) \ldots$ & $-14.2 \pm 0.2$ & This Paper & \\
\hline$\ldots$ & Spectral type ... & $\mathrm{F} 5 \pm 1$ & This Paper & \\
\hline$d$ & Distance $(\mathrm{pc}) \ldots$ & $263 \pm 14$ & This Paper & \\
\hline$\ldots$ & Age $(\mathrm{Gyr}) \ldots$ & $1.75 \pm 0.25$ & This Paper & \\
\hline & Visual extinction ... & $0.18 \pm 0.10$ & This Paper & \\
\hline$\left(U^{\mathrm{a}}, V, W\right)$ & Galactic space velocities $\left(\mathrm{km} \mathrm{s}^{-1}\right) \ldots$ & $(19.9 \pm 1.1,-9.6 \pm 0.5,-2.6 \pm 0.9)$ & This Paper & \\
\hline$M_{c z}$ & Mass of convective zone $\left(M_{\odot}\right) \ldots$ & {$[2.8 \pm 0.4] \times 10^{-4}$} & This Paper & \\
\hline$R_{c z}$ & Base of the convective zone $\left(R_{\odot}\right) \ldots$ & $1.31 \pm 0.03$ & This Paper & \\
\hline$C_{*}$ & Total moment of inertia (cgs) ... & {$[1.18 \pm 0.04] \times 10^{54}$} & This Paper & \\
\hline$C_{c z}$ & Convection zone moment of inertia $(\mathrm{cgs}) \ldots$ & {$[3.2 \pm 0.7] \times 10^{51}$} & This Paper & \\
\hline
\end{tabular}

Notes. (1) Høg et al. 1998; (2) Høg et al. 2000; (3) Skrutskie et al. 2006; Cutri et al. 2003, (4) Wright et al. 2010; Cutri et al. 2012. (5) Zacharias et al. 2004

${ }^{\text {a }}$ We adopt a right-handed coordinate system such that positive $U$ is toward the Galactic center.

Table 4

Median Values and 68\% Confidence Intervals for the Physical and Orbital Parameters of the KELT-1 System

\begin{tabular}{|c|c|c|c|}
\hline Variable & Description (Units) & Value $(e \neq 0)$ & Value $(e \equiv 0)$ \\
\hline \multicolumn{4}{|c|}{ Stellar parameters. } \\
\hline$M_{*} \ldots$ & Mass $\left(M_{\odot}\right) \ldots$ & $1.335 \pm 0.063$ & $1.332 \pm 0.063$ \\
\hline$R_{*} \ldots$ & $\operatorname{Radius}\left(R_{\odot}\right) \ldots$ & $1.471_{-0.035}^{+0.045}$ & $1.460_{-0.030}^{+0.039}$ \\
\hline$L_{*} \ldots$ & Luminosity $\left(L_{\odot}\right) \ldots$ & $3.51_{-0.21}^{+0.25}$ & $3.46_{-0.19}^{+0.23}$ \\
\hline$\rho_{*} \cdots$ & Density $(\mathrm{cgs}) \ldots$ & $0.594_{-0.042}^{+0.027}$ & $0.608_{-0.039}^{+0.019}$ \\
\hline $\log g \ldots$ & Surface gravity (cgs) ... & $4.228_{-0.021}^{+0.014}$ & $4.234_{-0.018}^{+0.012}$ \\
\hline$T_{\text {eff }} \ldots$ & Effective temperature $(\mathrm{K}) \ldots$ & $6516 \pm 49$ & $6516 \pm 49$ \\
\hline$[\mathrm{Fe} / \mathrm{H}] \ldots$ & Metallicity ... & $0.052 \pm 0.079$ & $0.052 \pm 0.079$ \\
\hline$v \sin I_{*} \ldots$ & Rotational velocity $\left(\mathrm{m} \mathrm{s}^{-1}\right)$ & $56000 \pm 2000$ & $56000 \pm 2000$ \\
\hline$\lambda \ldots$ & Spin-orbit alignment (degrees) . & $2 \pm 16$ & $1 \pm 15$ \\
\hline \multicolumn{4}{|c|}{ Planetary parameters. } \\
\hline$e \ldots$ & Eccentricity ... & $0.0100_{-0.0070}^{+0.010}$ & $\cdots$ \\
\hline$\omega_{*} \ldots$ & Argument of periastron (degrees) & $65_{-74}^{+72}$ & $\cdots$ \\
\hline$P \ldots$ & Period (days) $\ldots$ & $1.217513 \pm 0.000015$ & $1.217513 \pm 0.000015$ \\
\hline$a \ldots$ & Semimajor axis (AU) ... & $0.02472 \pm 0.00039$ & $0.02470 \pm 0.00039$ \\
\hline$M_{P} \ldots$ & Mass $\left(M_{\mathrm{J}}\right) \ldots$ & $27.38 \pm 0.93$ & $27.37 \pm 0.92$ \\
\hline$R_{P} \ldots$ & Radius $\left(R_{\mathrm{J}}\right) \ldots$ & $1.116_{-0.029}^{+0.038}$ & $1.108_{-0.025}^{+0.034}$ \\
\hline$\rho_{P} \ldots$ & Density $(\mathrm{cgs}) \ldots$ & $24.5_{-2.1}^{+1.5}$ & $25.0_{-1.9}^{+1.3}$ \\
\hline $\log g_{P} \ldots$ & Surface gravity ... & $4.736_{-0.025}^{+0.017}$ & $4.743_{-0.022}^{+0.014}$ \\
\hline$T_{e q} \ldots$ & Equilibrium temperature $(\mathrm{K}) \ldots$ & $2423_{-27}^{+34}$ & $2416_{-24}^{+30}$ \\
\hline$\Theta \ldots$ & Safronov number ... & $0.907_{-0.032}^{+0.028}$ & $0.915_{-0.028}^{+0.024}$ \\
\hline$\langle F\rangle \ldots$ & Incident flux $\left(10^{9} \mathrm{erg} \mathrm{s}^{-1} \mathrm{~cm}^{-2}\right) \ldots$ & $7.83_{-0.34}^{+0.45}$ & $7.73_{-0.30}^{+0.39}$ \\
\hline
\end{tabular}


Table 5

Median Values and 68\% Confidence Intervals for the Light Curve and Radial Velocity Parameters of the KELT-1 System

\begin{tabular}{|c|c|c|}
\hline Parameter & Description (Units) & Value \\
\hline \multicolumn{3}{|l|}{ RV parameters } \\
\hline$T_{C} \ldots$ & Time of inferior conjunction $\left(\mathrm{BJD}_{\mathrm{TDB}}\right) \ldots$ & $2455914.1628_{-0.0022}^{+0.0023}$ \\
\hline$T_{P} \ldots$ & Time of periastron $\left(\mathrm{BJD}_{\mathrm{TDB}}\right) \ldots$ & $2455914.08_{-0.25}^{+0.24}$ \\
\hline$K \ldots$ & RV semi-amplitude $\left(\mathrm{m} \mathrm{s}^{-1}\right) \ldots$ & $4239 \pm 52$ \\
\hline$K_{R} \ldots$ & RM amplitude $\left(\mathrm{m} \mathrm{s}^{-1}\right) \ldots$ & $343 \pm 13$ \\
\hline$M_{P} \sin i \ldots$ & Minimum mass $\left(M_{\mathrm{J}}\right) \ldots$ & $27.34 \pm 0.92$ \\
\hline$M_{P} / M_{*} \ldots$ & Mass ratio $\ldots$ & $0.01959 \pm 0.00040$ \\
\hline$u \ldots$ & RM linear limb darkening ... & $0.5861_{-0.0043}^{+0.0046}$ \\
\hline$\gamma_{0} \ldots$ & zero point for Orbital RVs (Table 7) $\left(\mathrm{m} \mathrm{s}^{-1}\right) \ldots$ & $-14200 \pm 49$ (stat.) \pm 200 (sys.) \\
\hline$\gamma_{1} \ldots$ & zero point for RM RVs (Table 8$)\left(\mathrm{m} \mathrm{s}^{-1}\right) \ldots$ & $-14200_{-59}^{+56}$ (stat.) \pm 200 (sys.) \\
\hline$e \cos \omega_{*} \ldots$ & 皮 & $0.0019_{-0.0060}^{+0.0091}$ \\
\hline$e \sin \omega_{*} \ldots$ & $\cdots$ & $0.0044_{-0.0063}^{+0.011}$ \\
\hline$f(m 1, m 2) \ldots$ & Mass function $\left(M_{\mathrm{J}}\right) \ldots$ & $0.01006 \pm 0.00037$ \\
\hline \multicolumn{3}{|l|}{ Transit parameters } \\
\hline$R_{P} / R_{*} \ldots$ & Radius of the planet in stellar radii ... & $0.07806_{-0.00058}^{+0.00061}$ \\
\hline$a / R_{*} \ldots$ & Semimajor axis in stellar radii ... & $3.619_{-0.087}^{+0.055}$ \\
\hline$i \ldots$ & Inclination (degrees) $\ldots$ & $87.6_{-1.9}^{+1.4}$ \\
\hline$b \ldots$ & Impact parameter ... & $0.150_{-0.088}^{+0.11}$ \\
\hline$\delta \ldots$ & Transit depth ... & $0.006093_{-0.000090}^{+0.000096}$ \\
\hline$T_{\text {FWHM }} \ldots$ & FWHM duration (days) ... & $0.10645 \pm 0.00045$ \\
\hline$\tau \ldots$ & Ingress/egress duration (days) $\ldots$ & $0.00873_{-0.00020}^{+0.00049}$ \\
\hline$T_{14} \ldots$ & Total duration (days) ... & $0.11526_{-0.00059}^{+0.00069}$ \\
\hline$P_{T} \ldots$ & A priori non-grazing transit probability ... & $0.2564_{-0.0055}^{+0.0079}$ \\
\hline$P_{T, G} \ldots$ & A priori transit probability ... & $0.2998_{-0.0065}^{+0.0093}$ \\
\hline$T_{C, 0} \ldots$ & transit time for PvdKO UT 2011 Dec 3 (BJD & $2455899.5549 \pm 0.0010$ \\
\hline$T_{C, 1} \ldots$ & transit time for ULMO UT 2011 Dec $3\left(\mathrm{BJD}_{\mathrm{TDB}}\right) \ldots$ & $2455899.55408 \pm 0.00044$ \\
\hline$T_{C, 2} \ldots$ & transit time for HAO UT 2011 Dec $10\left(\mathrm{BJD}_{\mathrm{TDB}}\right) \ldots$ & $2455905.63860_{-0.00082}^{+0.00004}$ \\
\hline$T_{C, 3} \ldots$ & transit time for FLWO UT 2011 Dec $16\left(\mathrm{BJD}_{\mathrm{TDB}}\right) \ldots$ & $2455911.72553 \pm 0.00045$ \\
\hline$T_{C, 4} \ldots$ & transit time for ULMO UT $2011 \mathrm{Dec} 31\left(\mathrm{BJD}_{\mathrm{TDB}}\right) \ldots$ & $2455927.55574_{-0.00042}^{+0.00040}$ \\
\hline$T_{C, 5} \ldots$ & transit time for FLWO UT 2012 Jan 7 (BJD $\left.\mathrm{BDB}_{\mathrm{TDB}}\right) \ldots$ & $2455933.64320_{-0.00039}^{+0.00041}$ \\
\hline$u_{1 \text { Sloani }} \cdots$ & linear limb-darkening coefficient ... & $0.2146_{-0.0042}^{+0.0046}$ \\
\hline$u_{2 \text { Sloani }} \ldots$ & quadratic limb-darkening coefficient ... & $0.3170_{-0.0033}^{+0.0038}$ \\
\hline$u_{1 \text { Sloanr }} \ldots$ & linear limb-darkening coefficient ... & $0.2865_{-0.0045}^{+0.0052}$ \\
\hline$u_{2 \text { Sloanr }} \ldots$ & quadratic limb-darkening coefficient ... & $0.3274_{-0.0026}^{+0.0032}$ \\
\hline$u_{1 \text { Sloanz }} \ldots$ & linear limb-darkening coefficient ... & $0.1646_{-0.0041}^{+0.0044}$ \\
\hline$u_{2 \text { Sloanz }} \cdots$ & quadratic limb-darkening coefficient ... & $0.3074_{-0.0028}^{+0.0031}$ \\
\hline \multicolumn{3}{|c|}{ Secondary eclipse parameters } \\
\hline$T_{S} \ldots$ & Time of eclipse $\left(\mathrm{BJD}_{\mathrm{TDB}}\right) \ldots$ & $2455913.5559_{-0.0050}^{+0.0063}$ \\
\hline$b_{S} \ldots$ & Impact parameter ... & $0.152_{-0.089}^{+0.12}$ \\
\hline$T_{S, \text { FWHM }} \ldots$ & FWHM duration (days) ... & $0.1074_{-0.0014}^{+0.0023}$ \\
\hline$\tau_{S} \ldots$ & Ingress/egress duration (days) ... & $0.00886_{-0.00029}^{+0.00052}$ \\
\hline$T_{S, 14} \ldots$ & Total duration (days) ... & $0.1164_{-0.0017}^{+0.0026}$ \\
\hline$P_{S} \ldots$ & A priori non-grazing eclipse probability ... & $0.2529_{-0.0026}^{+0.0059}$ \\
\hline$P_{S, G} \ldots$ & A priori eclipse probability ... & $0.2957_{-0.0031}^{+0.0071}$ \\
\hline
\end{tabular}

system is mounted atop a Paramount ME robotic mount from Software Bisque on a fixed pier at Winer Observatory in Sonoita, AZ (Latitude $31^{\circ} 39^{\prime} 56.08^{\prime \prime} \mathrm{N}$, Longitude $110^{\circ} 36^{\prime} 06.42^{\prime \prime} \mathrm{W}$, elevation $1515.7 \mathrm{~m})$. See Pepper et al. (2007) for additional details about the system hardware.

The primary KELT-North transit survey consists of 13 fields centered at +31.7 declination, spanning all $24 \mathrm{hr}$ of right ascension. Including the slight overlap between fields, the total survey area is $\approx 40 \%$ of the Northern sky. Survey observations consist of $150 \mathrm{~s}$ exposures with a typical per-field cadence of 15-30 minutes. The KELT-North telescope has been collecting survey data in this manner since 2006 September and to date has acquired between 5000 and 9300 images per field. Given this quantity of data and the typical achieved photometric precision of $\sim 1 \%$ for $V \lesssim 11$, the KELT-North survey is able to detect short-period giant transiting planets orbiting most FGK stars with magnitudes from saturation near $V \sim 8$ down to $V \sim 12$. 
Table 6

KELT-1 Transit Times

\begin{tabular}{lccccc}
\hline \hline Epoch & $\begin{array}{c}T_{C} \\
\text { BJD }_{\mathrm{TDB}}\end{array}$ & $\begin{array}{c}\sigma_{T_{C}} \\
(\mathrm{~s})\end{array}$ & $\begin{array}{c}O-C \\
(\mathrm{~s})\end{array}$ & $(O-C) / \sigma_{T_{C}}$ & Obs. \\
\hline-8 & 2455899.55496 & 88 & 187.11 & 2.11 & PvdKO \\
-8 & 2455899.55407 & 37 & 110.56 & 2.96 & ULMO \\
-3 & 2455905.63858 & 72 & -147.70 & -2.04 & HAO \\
2 & 2455911.72553 & 38 & -196.27 & -5.10 & FLWO \\
15 & 2455927.55574 & 35 & 37.52 & 1.05 & ULMO \\
20 & 2455933.64320 & 34 & 34.05 & 0.98 & FLWO \\
\hline
\end{tabular}

Table 7

KELT-1 Orbital Radial Velocities and Bisectors

\begin{tabular}{|c|c|c|c|c|}
\hline $\mathrm{BJD}_{\mathrm{TDB}}$ & $\begin{array}{c}\mathrm{RV} \\
\left(\mathrm{m} \mathrm{s}^{-1}\right)\end{array}$ & $\begin{array}{c}\sigma_{\mathrm{RV}} \\
\left(\mathrm{m} \mathrm{s}^{-1}\right)\end{array}$ & $\begin{array}{l}\text { Bisector } \\
\left(\mathrm{m} \mathrm{s}^{-1}\right)\end{array}$ & $\begin{array}{c}\sigma_{\mathrm{BS}} \\
\left(\mathrm{m} \mathrm{s}^{-1}\right)\end{array}$ \\
\hline 2455874.861355 & -10305.7 & 193.3 & 103.9 & 152.2 \\
\hline 2455876.751353 & -18142.8 & 182.9 & 121.5 & 106.7 \\
\hline 2455884.783908 & -10927.0 & 275.9 & 189.6 & 90.1 \\
\hline 2455885.723564 & -10514.3 & 238.3 & 56.0 & 140.3 \\
\hline 2455887.750071 & -18276.1 & 213.7 & 75.6 & 130.1 \\
\hline 2455888.709891 & -16515.4 & 173.4 & -61.1 & 117.0 \\
\hline 2455900.658271 & -11719.3 & 222.0 & -55.7 & 84.4 \\
\hline 2455901.773451 & -10631.5 & 366.7 & -41.6 & 108.3 \\
\hline 2455904.764448 & -18413.6 & 180.2 & -29.4 & 124.5 \\
\hline 2455905.598058 & -13110.9 & 190.2 & 127.0 & 74.8 \\
\hline 2455911.618841 & -11898.5 & 240.3 & 165.9 & 84.3 \\
\hline 2455912.633163 & -10246.8 & 325.6 & -33.0 & 160.8 \\
\hline 2455930.604440 & -13935.0 & 216.2 & 67.7 & 74.6 \\
\hline 2455931.588925 & -18254.2 & 210.1 & 131.8 & 46.3 \\
\hline 2455932.584867 & -17274.7 & 190.9 & -158.3 & 75.2 \\
\hline 2455934.572100 & -10040.1 & 248.2 & -53.4 & 82.5 \\
\hline 2455935.671466 & -11233.9 & 334.9 & -293.1 & 130.6 \\
\hline 2455936.607043 & -16403.1 & 307.3 & -34.7 & 209.3 \\
\hline 2455937.570378 & -18296.0 & 156.2 & 4.3 & 68.5 \\
\hline 2455940.565265 & -10305.4 & 224.9 & -210.3 & 102.9 \\
\hline 2455957.622238 & -10005.3 & 267.8 & -123.9 & 97.2 \\
\hline 2455960.657124 & -17866.6 & 297.0 & 10.2 & 171.4 \\
\hline 2455962.599920 & -9968.9 & 235.1 & 9.8 & 121.1 \\
\hline
\end{tabular}

Notes. Radial velocities and bisector spans with uncertainties for spectra taken with TRES at FLWO on nights outside of transit. Times are for mid-exposure and are in the BJD $_{\mathrm{TDB}}$ standard (Eastman et al. 2010). RVs are approximately on an absolute scale, and the uncertainties have been scaled based on a global fit to the data. See Sections 3.3 and 5.2 for details.

\subsection{KELT-North Pipeline}

Relative photometry is generated from flat-fielded images using the ISIS image subtraction package (Alard \& Lupton 1998; Alard 2000, see also Hartman et al. 2004), in combination with point-spread function (PSF) fitting using the stand-alone DAOPHOT II (Stetson 1987, 1990). Although highly effective, the image subtraction procedures are highly computer intensive. To improve reduction performance, the default ISIS scripts were modified to facilitate distributed image reduction across many computers in parallel. ISIS operation in this fashion permits thorough exploration of various reduction parameters, which would be intractable if executed serially. Other elements of the ISIS reduction package have also been modified or replaced with more robust alternatives. For example, the standard ISIS source-identification routines and utilities are ill equipped to deal with the nature and ubiquity of aberrations in KELT-North images. In response, we have replaced the ISIS "extract" utility with the popular SExtractor program (Bertin \& Arnouts 1996).
Table 8

KELT-1 RM Radial Velocities and Bisectors

\begin{tabular}{|c|c|c|c|c|}
\hline $\mathrm{BJD}_{\mathrm{TDB}}$ & $\begin{array}{c}\mathrm{RV} \\
\left(\mathrm{m} \mathrm{s}^{-1}\right)\end{array}$ & $\begin{array}{c}\sigma_{\mathrm{RV}} \\
\left(\mathrm{m} \mathrm{s}^{-1}\right)\end{array}$ & $\begin{array}{l}\text { Bisector } \\
\left(\mathrm{m} \mathrm{s}^{-1}\right)\end{array}$ & $\begin{array}{c}\sigma_{\mathrm{BS}} \\
\left(\mathrm{m} \mathrm{s}^{-1}\right)\end{array}$ \\
\hline 2455933.561465 & -12352.9 & 180.5 & 53.5 & 109.8 \\
\hline 2455933.565146 & -12544.0 & 215.7 & -178.0 & 141.5 \\
\hline 2455933.568838 & -12660.8 & 201.8 & 10.2 & 145.3 \\
\hline 2455933.573282 & -12565.8 & 190.2 & 218.3 & 130.1 \\
\hline 2455933.576973 & -12906.4 & 159.9 & 91.7 & 122.5 \\
\hline 2455933.580654 & -12948.6 & 214.2 & 11.1 & 151.7 \\
\hline 2455933.585237 & -12884.8 & 167.9 & -78.0 & 124.5 \\
\hline 2455933.588917 & -12709.2 & 152.7 & -211.0 & 149.3 \\
\hline 2455933.592609 & -12922.4 & 180.6 & -59.1 & 108.3 \\
\hline 2455933.596983 & -12893.1 & 154.8 & 50.0 & 134.5 \\
\hline 2455933.600664 & -12591.3 & 112.0 & -74.2 & 81.3 \\
\hline 2455933.604355 & -13057.7 & 190.5 & -34.8 & 113.6 \\
\hline 2455933.608765 & -13254.4 & 93.4 & 266.9 & 129.1 \\
\hline 2455933.612457 & -12858.8 & 135.4 & -4.4 & 122.2 \\
\hline 2455933.616137 & -13326.5 & 111.8 & 90.8 & 63.0 \\
\hline 2455933.621194 & -13524.8 & 152.7 & 148.1 & 114.1 \\
\hline 2455933.624875 & -13718.2 & 128.5 & 53.1 & 99.4 \\
\hline 2455933.628555 & -13756.6 & 139.9 & 19.5 & 137.3 \\
\hline 2455933.633034 & -13880.6 & 138.6 & 22.5 & 115.4 \\
\hline 2455933.636714 & -13844.8 & 115.0 & 177.2 & 130.4 \\
\hline 2455933.640406 & -14203.6 & 131.7 & 45.2 & 124.7 \\
\hline 2455933.645035 & -14292.8 & 142.4 & 0.4 & 82.1 \\
\hline 2455933.648715 & -14320.5 & 144.9 & -20.9 & 88.2 \\
\hline 2455933.652396 & -14319.4 & 172.8 & -188.0 & 86.4 \\
\hline 2455933.656869 & -14520.7 & 147.6 & 10.8 & 87.1 \\
\hline 2455933.660555 & -14793.0 & 136.8 & -234.2 & 104.5 \\
\hline 2455933.664235 & -14810.5 & 126.0 & -117.8 & 71.0 \\
\hline 2455933.668887 & -14923.9 & 135.6 & -183.6 & 88.4 \\
\hline 2455933.672567 & -15059.7 & 172.7 & 37.9 & 136.2 \\
\hline 2455933.676248 & -15021.1 & 103.3 & -21.6 & 112.4 \\
\hline 2455933.680761 & -15430.5 & 139.5 & 211.8 & 76.4 \\
\hline 2455933.684453 & -15333.1 & 180.1 & -82.2 & 198.6 \\
\hline 2455933.688133 & -15296.7 & 140.7 & 52.1 & 108.0 \\
\hline 2455933.692820 & -15561.8 & 155.9 & 164.2 & 135.4 \\
\hline 2455933.696501 & -15374.8 & 205.1 & -237.5 & 142.5 \\
\hline 2455933.700192 & -15283.4 & 311.1 & -699.1 & 412.4 \\
\hline 2455933.704648 & -15615.1 & 144.2 & 157.8 & 119.1 \\
\hline 2455933.708340 & -15314.0 & 217.0 & -54.8 & 133.8 \\
\hline 2455933.712020 & -15762.7 & 218.2 & 181.9 & 114.5 \\
\hline 2455933.716464 & -15772.2 & 218.7 & -206.9 & 147.1 \\
\hline 2455933.720145 & -16075.3 & 179.0 & -245.7 & 210.6 \\
\hline 2455933.723836 & -15779.8 & 181.1 & -33.8 & 160.4 \\
\hline
\end{tabular}

Notes. Radial velocities and bisector spans with uncertainties for spectra taken with TRES at FLWO on the night of the transit on UT 2012 January 7. Times are for mid-exposure and are in the BJD ${ }_{\mathrm{TDB}}$ standard (Eastman et al. 2010). RVs are approximately on an absolute scale, and the uncertainties have been scaled based on a global fit to the data. See Sections 3.3 and 5.2 for details.

A more complete explanation of these modifications and driver scripts that implement them are available online. ${ }^{19}$

\subsection{KELT-North Candidate Selection}

Once we have the light curves created by ISIS for all of the DAOPHOT-identified point sources in the reference image, we begin a series of post-processing steps before doing the initial candidate selection. To begin, we convert the ISIS light curves from differential flux to instrumental magnitude using the results of the DAOPHOT photometry on the reference image. We also apply $5 \sigma$ iterative clipping to all of the light curves at

\footnotetext{
19 http://astro.phy.vanderbilt.edu/ siverdrj/soft/is3/index.html
} 
Table 9

Relative Photometry from PvdKO on UT 2011 December 3 (i)

\begin{tabular}{lcc}
\hline \hline BJD $_{\mathrm{TDB}}$ & Relative Flux & Uncertainty \\
\hline 2455899.454080 & 1.00112 & 0.00177 \\
2455899.455342 & 0.99986 & 0.00177 \\
2455899.456603 & 0.99606 & 0.00206 \\
2455899.457865 & 0.99556 & 0.00218 \\
2455899.459138 & 1.00147 & 0.00192 \\
2455899.460399 & 0.99946 & 0.00163 \\
2455899.461661 & 0.99686 & 0.00162 \\
2455899.462934 & 1.00004 & 0.00190 \\
2455899.464207 & 1.00294 & 0.00221 \\
2455899.465468 & 0.99780 & 0.00175 \\
\hline
\end{tabular}

Notes. This photometry has been corrected for a linear trend with air mass, and normalized by the fitted out-of-transit flux. See Section 5.2.

(This table is available in its entirety in a machine-readable form in the online journal. A portion is shown here for guidance regarding its form and content.)

Table 10

Relative Photometry from ULMO on UT 2011 December 3 ( $r$ )

\begin{tabular}{lcc}
\hline \hline $\mathrm{BJD}_{\mathrm{TDB}}$ & Relative Flux & Uncertainty \\
\hline 2455899.475596 & 1.00155 & 0.00091 \\
2455899.476984 & 1.00058 & 0.00091 \\
2455899.478361 & 0.99852 & 0.00089 \\
2455899.479738 & 1.00015 & 0.00091 \\
2455899.481115 & 1.00175 & 0.00089 \\
2455899.482493 & 1.00039 & 0.00089 \\
2455899.483870 & 1.00020 & 0.00089 \\
2455899.485259 & 1.00144 & 0.00089 \\
2455899.486636 & 0.99967 & 0.00089 \\
2455899.488014 & 1.00099 & 0.00091 \\
\hline
\end{tabular}

Notes. This photometry has been corrected for a linear trend with air mass, and normalized by the fitted out-of-transit flux. See Section 5.2.

(This table is available in its entirety in a machine-readable form in the online journal. A portion is shown here for guidance regarding its form and content.)

Table 11

Relative Photometry from HAO on UT 2011 December 10 (i)

\begin{tabular}{lcc}
\hline \hline BJD $_{\text {TDB }}$ & Relative Flux & Uncertainty \\
\hline 2455905.540572 & 0.99833 & 0.00250 \\
2455905.541132 & 0.99667 & 0.00250 \\
2455905.541712 & 1.00321 & 0.00252 \\
2455905.542272 & 1.00209 & 0.00243 \\
2455905.542842 & 1.00116 & 0.00243 \\
2455905.543412 & 0.99931 & 0.00243 \\
2455905.543972 & 1.00078 & 0.00243 \\
2455905.544542 & 0.99829 & 0.00242 \\
2455905.545112 & 0.99994 & 0.00243 \\
2455905.545692 & 0.99800 & 0.00250 \\
\hline
\end{tabular}

Notes. This photometry has been corrected for a linear trend with air mass, and normalized by the fitted out-of-transit flux. See Section 5.2.

(This table is available in its entirety in a machine-readable form in the online journal. A portion is shown here for guidance regarding its form and content.)

this stage; this typically removes $\sim 0.6 \%$ of the data points. All of the uncertainties for the converted and clipped light curves in a given field are then scaled as an ensemble. The scaling is chosen such that the $\chi^{2} /$ dof $=1$ for the main locus of the light curves on a magnitude versus $\chi^{2} /$ dof plot. Typically this scaling is around a factor of 1.2, implying that the uncertainties are somewhat underestimated.
Table 12

Relative Photometry from FLWO on UT 2011 December $16(z)$

\begin{tabular}{lcc}
\hline \hline BJD $_{\text {TDB }}$ & Relative Flux & Uncertainty \\
\hline 2455911.612185 & 1.00109 & 0.00154 \\
2455911.612983 & 1.00230 & 0.00154 \\
2455911.613516 & 1.00009 & 0.00154 \\
2455911.614002 & 1.00177 & 0.00154 \\
2455911.614488 & 0.99899 & 0.00154 \\
2455911.615078 & 1.00118 & 0.00154 \\
2455911.615564 & 1.00159 & 0.00154 \\
2455911.616051 & 0.99953 & 0.00154 \\
2455911.616560 & 1.00155 & 0.00154 \\
2455911.617081 & 1.00066 & 0.00154
\end{tabular}

Notes. This photometry has been corrected for a linear trend with air mass, and normalized by the fitted out-of-transit flux. See Section 5.2.

(This table is available in its entirety in a machine-readable form in the online journal. A portion is shown here for guidance regarding its form and content.)

Table 13

Relative Photometry from ULMO on UT 2011 December 31 (r)

\begin{tabular}{lcc}
\hline \hline BJD \\
\hline 2455927.468704 & Relative Flux & Uncertainty \\
2455927.470335 & 0.99844 & 0.00145 \\
2455927.471713 & 0.99820 & 0.00125 \\
2455927.473090 & 0.99925 & 0.00116 \\
2455927.474467 & 1.00005 & 0.00105 \\
2455927.475844 & 0.99994 & 0.00099 \\
2455927.477233 & 1.00105 & 0.00092 \\
2455927.478610 & 0.99940 & 0.00090 \\
2455927.479987 & 1.00084 & 0.00086 \\
2455927.481365 & 0.99960 & 0.00086 \\
\hline
\end{tabular}

Notes. This photometry has been corrected for a linear trend with air mass, and normalized by the fitted out-of-transit flux. See Section 5.2.

(This table is available in its entirety in a machine-readable form in the online journal. A portion is shown here for guidance regarding its form and content.)

Table 14

Relative Photometry from FLWO on UT 2012 January 7 (i)

\begin{tabular}{lcc}
\hline \hline BJD $_{\mathrm{TDB}}$ & Relative Flux & Uncertainty \\
\hline 2455933.568776 & 1.00164 & 0.00184 \\
2455933.569101 & 0.99893 & 0.00184 \\
2455933.569459 & 1.00032 & 0.00184 \\
2455933.569830 & 1.00046 & 0.00184 \\
2455933.570200 & 0.99947 & 0.00184 \\
2455933.570536 & 1.00032 & 0.00184 \\
2455933.570871 & 1.00131 & 0.00184 \\
2455933.571195 & 1.00074 & 0.00184 \\
2455933.571531 & 0.99764 & 0.00184 \\
2455933.571867 & 1.00003 & 0.00184 \\
\hline
\end{tabular}

Notes. This photometry has been corrected for a linear trend with air mass, and normalized by the fitted out-of-transit flux. See Section 5.2.

(This table is available in its entirety in a machine-readable form in the online journal. A portion is shown here for guidance regarding its form and content.)

We next attempt to match all of the DAOPHOT-identified point sources in the reference image to stars in the Tycho-2 catalog. We obtain a full-frame WCS with sub-pixel accuracy on our reference frame using Astrometry.net (Lang et al. 2010). Using this solution, we match stars by taking the closest Tycho-2 entry within $45^{\prime \prime}$. This typically generates matches for $98 \%$ of the Tycho-2 stars within each field. A successful Tycho-2 match 
Table 15

Relative Photometry from ULMO on UT 2011 December 2 (i)

\begin{tabular}{lcc}
\hline \hline BJD $_{\text {TDB }}$ & Relative Flux & Uncertainty \\
\hline 2455897.65499 & 1.00097 & 0.00145 \\
2455897.65721 & 0.99944 & 0.00134 \\
2455897.65859 & 0.99993 & 0.00140 \\
2455897.65997 & 0.99930 & 0.00141 \\
2455897.66134 & 1.00078 & 0.00140 \\
2455897.66273 & 1.00023 & 0.00138 \\
2455897.66411 & 1.00137 & 0.00134 \\
2455897.66549 & 0.99971 & 0.00138 \\
2455897.66686 & 0.99978 & 0.00140 \\
2455897.66824 & 0.99970 & 0.00140 \\
\hline
\end{tabular}

Notes. This photometry has been normalized and corrected for a linear trend with time. See Section 5.4.

(This table is available in its entirety in a machine-readable form in the online journal. A portion is shown here for guidance regarding its form and content.)

Table 16

Relative Photometry from FTN/LCOGT on UT 2011 December 30 (PS-Z)

\begin{tabular}{lcc}
\hline \hline BJD $_{\text {TDB }}$ & Relative Flux & Uncertainty \\
\hline 2455925.730250 & 0.99855 & 0.00078 \\
2455925.731230 & 1.00045 & 0.00078 \\
2455925.732210 & 0.99953 & 0.00078 \\
2455925.733190 & 1.00032 & 0.00078 \\
2455925.734190 & 1.00069 & 0.00078 \\
2455925.735170 & 1.00000 & 0.00078 \\
2455925.739770 & 1.00089 & 0.00078 \\
2455925.740700 & 0.99925 & 0.00078 \\
2455925.741670 & 0.99874 & 0.00078 \\
2455925.742660 & 0.99933 & 0.00078 \\
\hline
\end{tabular}

Notes. This photometry has been normalized and corrected for a linear trend with time. See Section 5.4.

(This table is available in its entirety in a machine-readable form in the online journal. A portion is shown here for guidance regarding its form and content.)

also will provide a Two Micron All Sky Survey (2MASS) ID. We use the proper motions and $J H K$ apparent magnitudes from these two catalogs.

With this catalog information, we next identify and exclude giant stars by means of a reduced proper motion $\left(H_{J}\right)$ diagram (Gould \& Morgan 2003). Following the specific prescription of Collier Cameron et al. (2007b), we place each of our matched stars on a $J$ versus $H_{J}$ plot. We compute the reduced proper motion of a star as

$$
H_{J}=J+5 \log \left(\mu / \operatorname{mas}_{\mathrm{yr}^{-1}}\right)
$$

and determine the star to be a giant if it fails to satisfy

$$
\begin{aligned}
H_{J}> & -141.25(J-H)^{4}+473.18(J-H)^{3} \\
& -583.6(J-H)^{2}+313.42(J-H)-43.0 .
\end{aligned}
$$

This process leaves us with anywhere from 10,000 to 30,000 catalog-matched putative dwarf stars and subgiants (hereafter dwarfs) per field, depending primarily on the location of the field relative to the Galactic plane.

The dwarfs are then run through the Trend Filtering Algorithm (TFA; Kovács et al. 2005) ${ }^{20}$ to reduce systematic noise. We

\footnotetext{
20 We used the versions of TFA and BLS (described later) found in the VARTOOLS package (Hartman et al. 2008).
}

Table 17

Relative Photometry from ULMO on UT 2012 January 4 (i)

\begin{tabular}{lcc}
\hline \hline BJD $_{\text {TDB }}$ & Relative Flux & Uncertainty \\
\hline 2455930.50268 & 1.00020 & 0.00111 \\
2455930.50487 & 1.00126 & 0.00110 \\
2455930.50624 & 0.99911 & 0.00110 \\
2455930.50762 & 0.99970 & 0.00111 \\
2455930.50901 & 1.00139 & 0.00116 \\
2455930.51039 & 1.00146 & 0.00111 \\
2455930.51177 & 0.99958 & 0.00111 \\
2455930.51314 & 1.00016 & 0.00111 \\
2455930.51452 & 1.00098 & 0.00111 \\
2455930.51590 & 1.00085 & 0.00111 \\
\hline
\end{tabular}

Notes. This photometry has been normalized and corrected for a linear trend with time. See Section 5.4.

(This table is available in its entirety in a machine-readable form in the online journal. A portion is shown here for guidance regarding its form and content.)

select a new set of detrending stars for each light curve by taking the 150 closest stars - outside of a 20 pixel exclusion zone centered on the star being detrended-that are within two instrumental magnitudes of the star being detrended.

KELT's Paramount ME is a German Equatorial mount, which requires a "flip" as it tracks stars past the meridian. Therefore, the optics and detector are rotated $180 \mathrm{deg}$ with respect to the stars between observations in the Eastern and Western hemispheres, and detector defects, optical distortions, PSF shape, flat-fielding errors, etc., for a given star can be completely different. This requires us to treat observations in the East and West essentially as two separate instruments. Thus, the preceding steps (magnitude conversion, error scaling, dwarf identification, TFA) are each performed separately on the East and West images of each field. After the dwarf stars in the East and West have been run through TFA, we then combine the two light curves of each target into one East+West light curve. We first match stars from the East and the West pointings by their Tycho IDs, and then determine the error-weighted scaling factor of the Western light curve needed to match the error-weighted mean instrumental magnitude of the East light curve.

All of the light curves from the matched Tycho dwarf stars in a field are given an internal ID. We next search the combined East+West light curves of the dwarfs for transit-like signals using the box-fitting least-squares algorithm (BLS; Kovács et al. 2002). We use a custom version of BLS modified to skip over integer and half-integer multiples of the sidereal day to reduce the effect of spurious signals due to diurnal systematics and their aliases on the BLS statistics. We perform selection cuts along six of the statistics that are output by the VARTOOLS implementation of the BLS algorithm: signal detection efficiency SDE, signal to pink noise SPN, ${ }^{21}$ the fraction of transit points from one night $f_{1 n}$, depth $\delta$, the ratio of $\Delta \chi^{2}$ for the best transit model to best inverse transit model $\Delta \chi^{2} / \Delta \chi_{-}^{2}$ (Burke et al. 2006), and the fraction of the orbit spent in transit or duty cycle $q$. In order to determine the appropriate threshold values for these statistics, we injected realistic transit signals with a range of properties into a large sample of light curves, and then attempted to recover these using the BLS algorithm. We then determined the values of these statistics that roughly maximize the overall detection efficiency while minimizing the number of spurious detections. The final adopted values are given in Table 1. 21 See Kovács et al. (2002) and Hartman et al. (2009), respectively, for the
definitions of SDE and SPN. 
In addition to the cuts we make on the BLS statistics, we also impose restrictions on the effective temperature and inferred density of the candidate host stars. For the temperature, we require that $T_{\text {eff }}<7500 \mathrm{~K}$. We calculate the stellar effective temperature of each candidate from its 2MASS $J-K$ colors. We used the Yonsei-Yale isochrones (Demarque et al. 2004) at 5 Gyr with solar metallicity and no alpha enhancement to create a simple polynomial fit for $T_{\text {eff }}$ as a function of $J-K$ :

$$
\begin{aligned}
\log T_{\mathrm{eff}}= & 3.94808-0.7353(J-K) \\
& +1.0116(J-K)^{2}-0.8334(J-K)^{3} .
\end{aligned}
$$

As we have conducted our follow-up spectroscopy, we have found that this relation generally predicts $T_{\text {eff }}$ to within $\sim 100 \mathrm{~K}$ for $T_{\text {eff }} \lesssim 7000 \mathrm{~K}$ and to within $\sim 300 \mathrm{~K}$ for stars with $T_{\text {eff }}=$ $7000-7500 \mathrm{~K}$.

We also require that the stellar density, $\rho_{*}$, as inferred from the BLS transit fits to the KELT-North light curve, to be within 1.0 dex of the stellar density calculated for each star using its $J-K$ colors, assuming the star is on the main sequence. A large disparity in the observed versus the calculated density is indicative of a blend or of a giant that made it through the reduced proper motion cuts (Seager \& Mallén-Ornelas 2003). Again using the Yonsei-Yale isochrones at $5 \mathrm{Gyr}$ with solar metallicity and no alpha enhancement, we made a fit for density as a function of $J-K$ :

$$
\begin{aligned}
\log \left(\rho_{*, \text { calc }} / \rho_{\odot}\right)= & -1.00972+2.82824(J-K) \\
& -1.19772(J-K)^{2} .
\end{aligned}
$$

We require that this value be within 1.0 dex of the stellar density we calculate from the KELT-North light curve

$$
\log \rho_{*, \mathrm{obs}}=\log \left[\frac{3}{G \pi^{2} q^{3} P^{2}}\right],
$$

where $P$ and $q$ are the orbital period and duty cycle (transit duration relative to the period) as returned by BLS. This equation assumes circular orbits and that the companion mass is much smaller than the host star mass, $M_{P} \ll M_{*}$. Also, because BLS does not attempt to fit for the ingress/egress duration, and furthermore KELT-North data typically do not resolve the ingress or egress well, we are not able to determine the transit impact parameter and thus the true stellar radius crossing time. Equation (5) therefore implicitly assumes an equatorial transit, and so formally provides only an upper limit to the true stellar density. For a transit with an impact parameter of $b=0.7$, the true density is $\sim 0.5$ dex smaller than that inferred from Equation (5).

All of the light curves that pass these selection criteria are designated as candidates, and a number of additional diagnostic tests are then performed on them, including Lomb-Scargle (LS, Lomb 1976; Scargle 1982) and AoV (Schwarzenberg-Czerny 1989; Devor 2005) periodograms. The results of these tests, the values of the BLS statistics, the light curves themselves, as well as a host of additional information, are all collected into a Web site for each candidate. Members of the team can then use this information to vote on the true nature of the candidate (probable planet, eclipsing binary, sinusoidal variable, spurious detection, blend, or other). All candidates with at least one vote for being a probable planet are then discussed, and the most promising are then passed along for follow-up photometry, reconnaissance spectroscopy, or both.

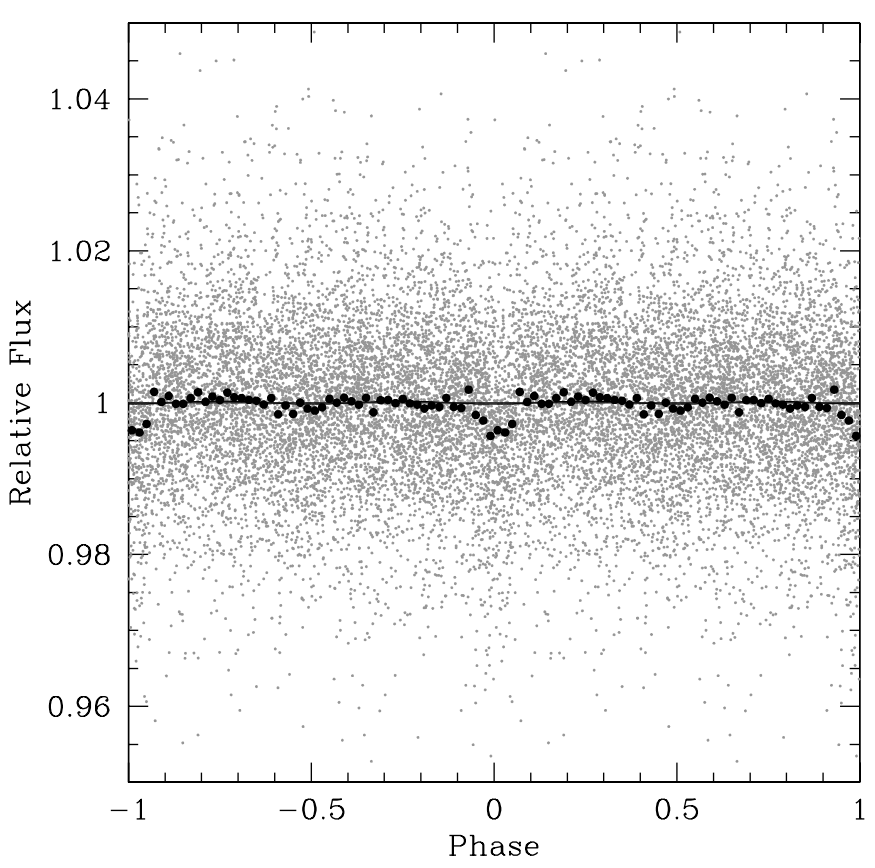

Figure 1. KELT-North light curve of KELT-1 phased to the BLS determined period of $P=1.2175$ days is shown in the gray points. The black points show the data binned 0.02 in phase.

\section{OBSERVATIONS}

\subsection{KELT-North Photometry, Candidate Identification, and Vetting Overview}

KC20C05168 emerged as a strong candidate from the analysis of the combined light curves from stars in the overlap area between fields 1 and 13. The KC20C05168 light curve contains 8185 epochs distributed over $\sim 4.2$ years, between UT 2006 October 25 and UT 2010 December 28, with a weighted rms of 9.8 millimagnitudes (mmag). This rms is typical for KELTNorth light curves of stars with this magnitude $(V \sim 10.7)$. A strong BLS signal was found at a period of $P \simeq 1.2175$ days, with a depth of $\delta \simeq 3.8 \mathrm{mmag}$, and detection statistics SPN $=$ 8.53, $\mathrm{SDE}=12.41, q=0.09, \Delta \chi^{2} / \Delta \chi_{-}^{2}=2.06$, and $\log \left(\rho_{*, \text { obs }} / \rho_{*, \text { cal }}\right)=-0.06$. The phased KELT-North light curve is shown in Figure 1. A significant signal also appeared in SuperWASP data (Butters et al. 2010) of this star at the same period. The KELT-North data exhibit some evidence for out-oftransit variability at the mmag level and exhibit some relatively weak peaks in the LS and AoV periodograms, but we did not consider these signals to be strong enough to warrant rejection of the candidate. In addition, the depth of the photometric transit signal in the original KELT-North light curve is substantially smaller than we find in the high-precision follow-up data (see Section 3.4). Further analysis indicates that the out-of-transit variability and smaller depth were likely due to a minor problem with the original data reduction.

Based on the strength of the K20C05168 signal, the estimated effective temperature of the host star of $T_{\text {eff }} \sim 6500 \mathrm{~K}$, and the fact that the star was sufficiently isolated in a DSS image, we submitted the candidate for reconnaissance spectroscopy with the Tillinghast Reflector Echelle Spectrograph (TRES; Fürész 2008) on the $1.5 \mathrm{~m}$ Tillinghast Reflector at the Fred Lawrence Whipple Observatory (FLWO) on Mount Hopkins in Arizona. The first observation on UT 2011 November 9 at the predicted quadrature confirmed the $T_{\text {eff }}$ estimate of the star, and also 
demonstrated that it was a slightly evolved dwarf with $\log g \sim 4$, and that it was rapidly rotating with $v \sin I_{*} \sim 55 \mathrm{~km} \mathrm{~s}^{-1}$. A second observation was obtained on UT 2011 November 11 separated by $\sim 1.9$ days or $\sim 1.54$ in phase, from the first observation and thus sampled the opposite quadrature. The two observations exhibited a large and significant RV shift of $\sim 8 \mathrm{~km} \mathrm{~s}^{-1}$, consistent with a BD companion.

Efforts to obtain photometric follow-up during the transit and secondary eclipse were then initiated. Concurrently, additional spectra with TRES were taken to characterize the spectroscopic orbit. In addition, we obtained adaptive optics imaging of the target to search for close companions. Finally, once we were fairly confident that the signals were due to a low-mass transiting companion, we obtained continuous spectroscopic time series with TRES during the transits on UT 2011 December 21 and UT 2012 January 7 for the purposes of measuring the Rossiter-McLaughlin (RM) effect. All of these observations are described in greater detail in the subsequent sections and summarized in Table 2.

\subsection{Previous Identification of the Photometric Candidate by HATNet}

KELT-1b was also recognized as a photometric transiting planet candidate by the HATNet project, based on observations obtained in 2006. In 2009 September the candidate HTR162002 was forwarded to D. Latham's team for spectroscopic follow-up. An initial observation with TRES confirmed that the target was a late $\mathrm{F}$ main-sequence star, as expected from 2MASS color $J-K_{s}=0.245$. The synthetic template with $T_{\text {eff }}=6250 \mathrm{~K}$ and $\log g=4.0$ and assumed solar metallicity gave the best match to the observed spectrum. However, that first TRES spectrum also revealed that the star was rotating rapidly, with $v \sin I_{*}=55 \mathrm{~km} \mathrm{~s}^{-1}$. At that time, the D. Latham's team routinely put aside candidates rotating more rapidly than about $v \sin I_{*}=30 \mathrm{~km} \mathrm{~s}^{-1}$, arguing that it would not be possible to determine velocities with a precision sufficient for an orbital solution for a planetary companion.

HTR162-002 remained on the HATNet "do not observe with TRES" list until it was independently rediscovered by the KELTNorth team and was forwarded as candidate KC20C05168 to D. Latham's team in 2011 November for spectroscopic follow-up with TRES. During the intervening 26 months, there were two relevant developments in the procedures and tools used by Latham's team, both resulting from contributions by L. Buchhave. The first development, enabled by convenient tools in the observing Web site, was the practice of observing new candidates only near opposite quadratures, according to the discovery ephemeris and assuming circular orbits. The second development was a much improved procedure for deriving radial velocities for rapidly rotating stars, initially motivated by the Kepler discovery of hot white dwarfs transiting rapidly rotating A stars (Rowe et al. 2010). As it turned out, the second observation of KC20C05168 with TRES described above was taken before the first observation was reviewed, so the candidate was not relegated to the rejected list due to its rapid rotation before the opposite quadrature was observed. When the results were reviewed after the second observation, the evidence for a significant RV shift between the two observations was obvious, despite the rapid rotation, therefore suggesting that the unseen companion was probably a $\mathrm{BD}$, if not a giant planet.

It should also be recognized that over the 26 months since the first observation of HTR162-002, the attitude against pursuing rapidly rotating stars as possible hosts for transiting planets had gradually softened among the exoplanet community. An important development was the demonstration that slowly rotating subgiants that have evolved from rapidly rotating mainsequence A stars do occasionally show the RV signatures of orbiting planetary companion (e.g., Johnson et al. 2007). A second insight came from the demonstration that the companion that transits the rapidly rotating A star WASP-33 must be a planet, using Doppler imaging (Collier Cameron et al. 2010). Finally, the discovery of transiting BD companions suggested the possibility of detecting their large amplitude RV signals even when they orbit stars with large $v \sin I_{*}$ and thus poor RV precision.

In the early days of wide-angle photometric surveys for transiting planets, Latham's team had established procedures for handling candidates forwarded for spectroscopic follow-up by more than one team. Such duplications were fairly common, and the goal was to assign credit to the initial discovery team, which was especially important in an era when few transiting planets had been confirmed. By the time it was noticed in mid 2011 December that KC20C05168 was the same as HTR162-002, the KELT-North team already had in hand a convincing orbital solution from TRES and high-quality light curves from several sources, confirming that KELT-1b was indeed a substellar companion.

\subsection{Spectroscopy from FLWO/TRES}

A total of 81 spectra of KELT-1 were taken using the TRES spectrograph on the $1.5 \mathrm{~m}$ Tillinghast Reflector at FLWO. These were used to determine the Keplerian parameters of the spectroscopic orbit, measure bisector variations in order to exclude false positive scenarios, measure the spectroscopic parameters of the primary, and measure anomalous RV shift of the stellar spectral lines as the companion transits in front of the rapidly rotating host star, i.e., the RM effect (Rossiter 1924; McLaughlin 1924). The TRES spectrograph provides high resolution, fiber-fed echelle spectroscopy over a bandpass of 3900-8900 Å (Fürész 2008). The observations obtained here employed the medium fiber for a resolution of $R \sim 44,000$. The data were reduced and analyzed using the methods described in Quinn et al. (2012) and Buchhave et al. (2010).

A subset of six spectra were combined in order to determine the spectroscopic parameters of the host star using the Spectral Parameter Classification (SPC) fitting program (L. A. Buchhave et al., in preparation). SPC cross-correlates the observed spectrum against a grid of synthetic Kurucz (Kurucz 1979) spectra. This analysis yielded $T_{\text {eff }}=6512 \pm 50 \mathrm{~K}, \log g=4.20 \pm 0.10$, $[\mathrm{Fe} / \mathrm{H}]=0.06 \pm 0.08$, and $v \sin I_{*}=55.2 \pm 2.0 \mathrm{~km} \mathrm{~s}^{-1}$. These parameters were used as priors for the joint global fit to the RV, RM, and photometric data as described in Section 5.2.

Spectra were taken at a range of phases in order to characterize the spectroscopic orbit and search for bisector span variations indicative of a blend. One of these spectra happened to be taken during a transit on UT 2011 November 18, and so was not used in the analysis because it is likely to be affected by the RM effect. The RV and bisector data for the remaining 23 spectra are listed in Table 7. These observations span $\sim 88$ days from UT 2011 November 9 through UT 2012 February 5. The uncertainties on the listed radial velocities have been scaled by a factor of 1.214 based on an independent fit to these data, as described in Section 5.2. The scaled median RV uncertainty is $\sim 230 \mathrm{~m} \mathrm{~s}^{-1}$. The uncertainties in the bisector measurements have not been scaled. The median bisector uncertainty is $\sim 110 \mathrm{~m} \mathrm{~s}^{-1}$. 


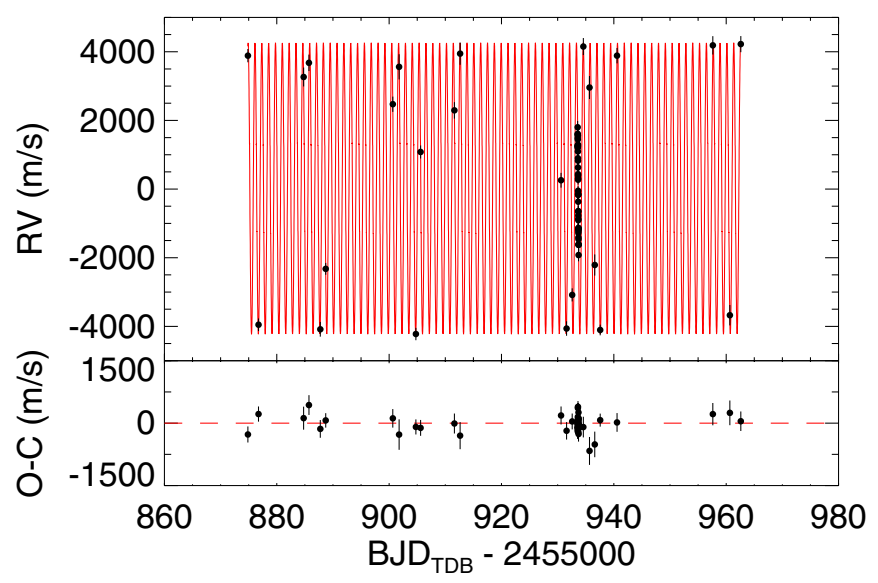

Figure 2. Top panel: the points with uncertainties show the measured RVs for

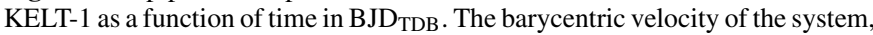
as determined from the model fit shown in the solid line (see Section 5.2), has been subtracted from the data. Bottom panel: the residuals from the model fit. (A color version of this figure is available in the online journal.)

Time series spectroscopy was obtained with TRES on two different nights of transits in order to measure the spin-orbit alignment of the companion via the RM effect. On UT 2011 December 2115 observations were obtained and 42 observations on UT 2012 January 7. Conditions were relatively poor for the first run, resulting in a factor $\sim 2$ larger uncertainties and incomplete coverage of the transit. We therefore decided not to include these data in our final analysis, although we confirmed that this has no effect on our final inferred parameters. The RV and bisector data for the RM run on UT 2012 January 7 are listed in Table 8 . The RV uncertainties have been scaled by a factor of 0.731 , also based on the global model fit described in Section 5.2. We note that the majority of the $\chi^{2}$ for these data are due to a few outliers. The median scaled $\mathrm{RV}$ uncertainty is $\sim 160 \mathrm{~m} \mathrm{~s}^{-1}$. The bisector uncertainties were not scaled.

All of the RV measurements used in the subsequent analysis are shown as a function of epoch of observation in $\mathrm{BJD}_{\mathrm{TDB}}$ in Figure 2. The RV and bisector measurements phased to the bestfit companion period from the joint fit to photometric and RV data are shown in Figure 3, demonstrating the very high signalto-noise ratio with which the RV signal of the companion was detected, and the good phase coverage of the orbit. A detail of the RV data near the time transit with the orbital (Doppler) RV signal removed is shown in Figure 4, showing the clear detection of the RM effect and a suggestion that the orbit normal is well aligned with the projected stellar spin axis.

Finally, we determined the absolute RV of the system barycenter using a simple circular orbit fit to radial velocities determined from the full set of spectra, which were determined using a single order near the $\mathrm{Mg} \mathrm{b}$ line. (Note that the relative RVs used for determining the orbit were determined using the full, multi-order analysis of the spectra.) The zero point correction to these velocities was determined using contemporaneous monitoring of five RV standard stars. The final value we obtain is $\gamma_{\mathrm{obs}}=-14.2 \pm 0.2 \mathrm{~km} \mathrm{~s}^{-1}$, where the uncertainty is dominated by the systematic uncertainties in the absolute velocities of the standard stars. This zero point, along with the global fit to the data in Section 5.2, was used to place the instrumental relative radial velocities on an absolute scale. Therefore, the RVs listed in Tables 7 and 8 are on an absolute scale.

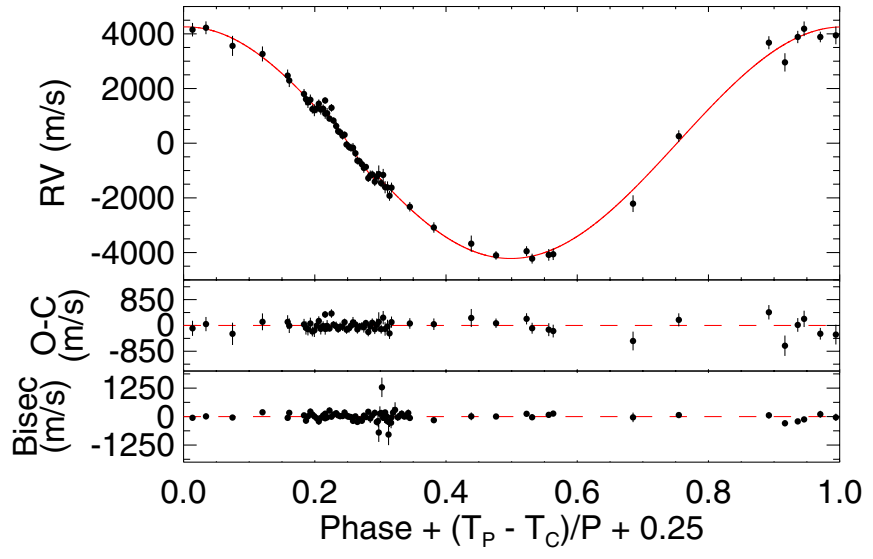

Figure 3. Points with uncertainties show the measured RVs for KELT-1 relative to the barycentric velocity of the system, phased to the best-fit period as determined from the model fit shown in the solid line (see Section 5.2). The phases are normally referenced to the time of periastron $\left(T_{P}\right)$, but have been shifted such that a phase of 0.25 corresponds to the time of inferior conjunction $T_{C}$ or transit. RV data near this phase show deviations from the Keplerian expectation due to the RM effect, which was included in the model. Middle panel: the residuals of the RV data from the model fit. Bottom panel: bisector spans as a function of phase.

(A color version of this figure is available in the online journal.)

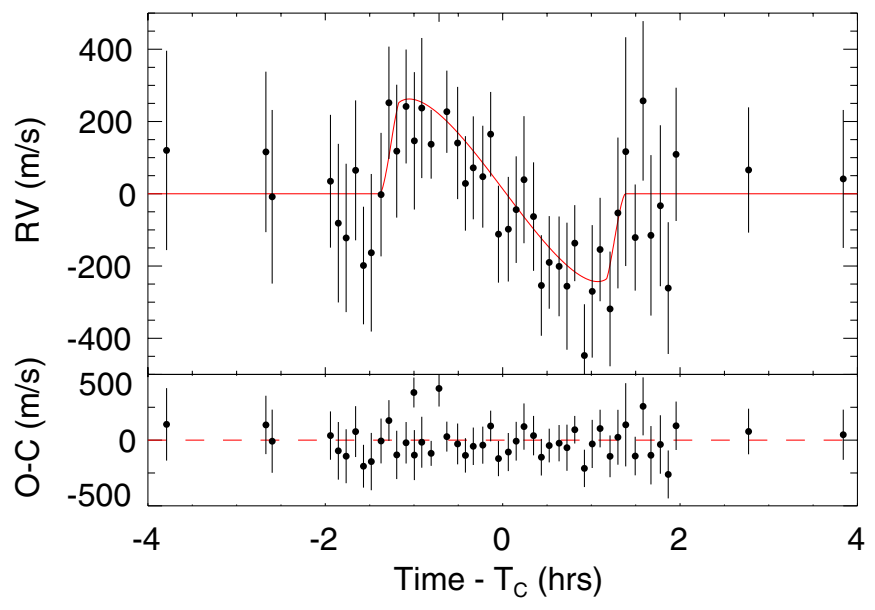

Figure 4. Top panel: the points with uncertainties show the measured RVs relative to the barycentric velocity of the system for KELT-1, as a function of the time since transit $T_{C}$, for data taken near $T_{C}$. The Keplerian RV variation as determined from the best-fit model has been removed from both the data and model. Data taken within $\sim 1.4 \mathrm{hr}$ of $T_{C}$ occur during transit and are thus strongly affected by the RM effect. The shape of the RM signal indicates that the projected obliquity of the host star with respect to the orbit is small. Bottom panel: the residuals of the data to the RM fit.

(A color version of this figure is available in the online journal.)

\subsection{Follow-up Photometry}

We obtained high-precision follow-up photometry of KELT-1 in order to confirm the K20C05168 transit signal, search for evidence of a strongly wavelength-dependent transit depth indicative of a stellar blend, and search for evidence of a secondary eclipse. Also, these data enable precision measurements of the transit depth, ingress/egress duration, and total duration, in order to determine the detailed parameters of the KELT-1 system. In all, we obtained coverage of nine complete and four partial transits, and two complete and one partial secondary eclipse, using six different telescopes in all. Many of these data were taken under relatively poor conditions and/or suffer from strong systematics. We therefore chose to include only a subset for the final 


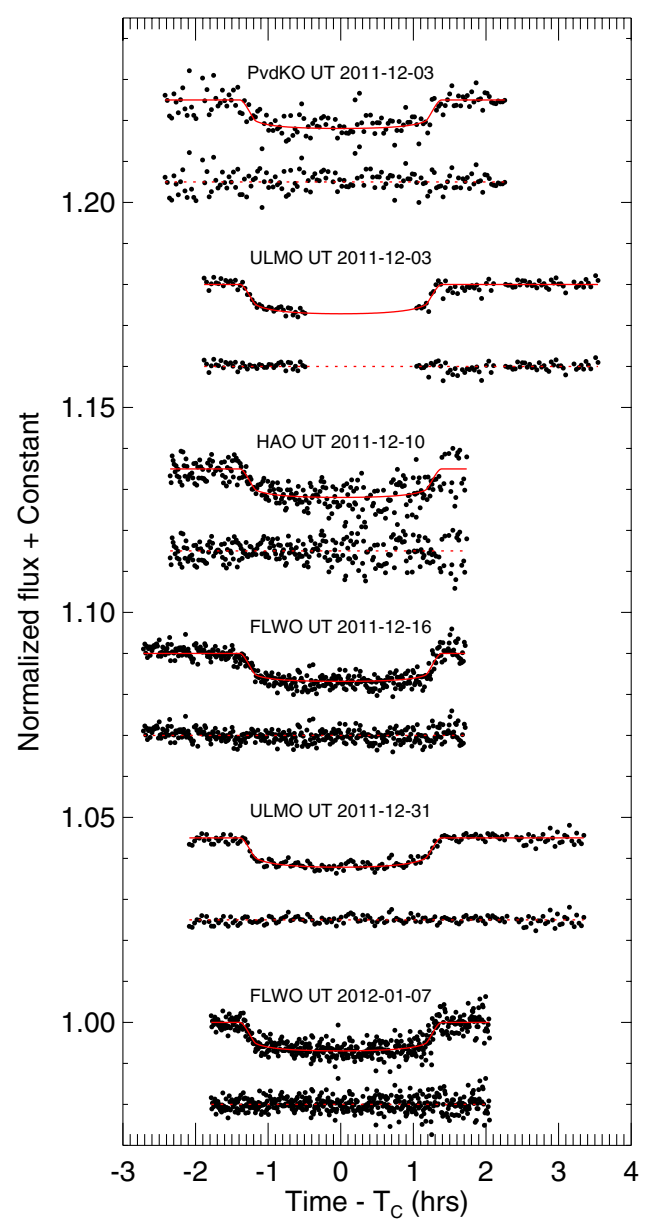

Figure 5. Points show the relative flux as a function of time from transit $\left(T_{C}\right)$ for the six sets of follow-up observations of transits we analyze here. The data sets are labeled and summarized in Table 2. The data are normalized by the fitted out-of-transit flux, and a linear trend with air mass has been removed (see Section 5.2). In addition, an arbitrary offset has been applied to each light curve for clarity. For each observation, we plot the data above and the residuals below. In all cases, the solid lines show the model fit from the analysis in Section 5.2.

(A color version of this figure is available in the online journal.)

analysis, including six of the transits and the three secondary eclipses. In the following subsections, we detail the observatories and data reduction methods used to obtain these data. The dates, observatories, and filters for these data sets are summarized in Table 2. The light curves for the transits are displayed in Figure 5 and the data are listed in Tables 9-14, whereas the light curves for the secondary eclipse are displayed in Figure 6 and the data are listed in Tables 15-17. The combined and binned transit light curve is shown in Figure 7.

\subsubsection{Peter van de Kamp Observatory $(P v d K O)$}

Data on the transit starting on UT 2011 December 3 were acquired with the $0.6 \mathrm{~m}, f / 7.8$ Ritchey-Chrétien telescope at the Peter van de Kamp Observatory at Swarthmore College (Swarthmore, PA, USA). The telescope is equipped with an Apogee U16M CCD with $4096 \times 40969 \mu \mathrm{m}$ pixels, giving a field of view 26 arcmin on a side. Available filters are $50 \mathrm{~mm}$ square Johnson-Cousins $U B V R_{c} I_{c}$ and SDSS ugriz, both from Omega Optical. The telescope is autoguided to minimize photometric errors from imperfect flat fielding, keeping the centroid shift of each star to within typically 3-4 pixels over the course of a night. The observations used here were obtained with the $i$ filter and used $2 \times 2$ binning, giving a binned pixel scale of 0.76 pixel $^{-1}$.

The data were reduced in IRAF using standard procedures for flat-fielding (with twilight sky flats) and dark and bias subtraction. Aperture photometry was performed, and then differential magnitudes for the target star were calculated using an ensemble of comparison stars in the same field, chosen to minimize the scatter in the final light curve.

\subsubsection{University of Louisville Moore Observatory (ULMO)}

Data on the transits starting UT 2011 December 3 and 2011 December 31, and on the secondary eclipses starting 2011 December 2 and 2012 January 4, were obtained with the University of Louisville Moore Observatory RC24 telescope (MORC24) located near Brownsboro, Kentucky. MORC24 is an RC Optical Systems Ritchey-Chrétien $0.6 \mathrm{~m}$ telescope on an equatorial fork mount. The telescope is equipped with an Apogee U16M $4096 \times 4096$ pixel CCD camera which has a focal plane scale of 0 '.39 pixel $^{-1}$ and a field of view (FOV) of $26.3 \times 26 ! 3$. The UT 2011 December 3 and 2011 December 31 data were obtained using an Astrodon Photometrics Sloan $r$ filter, while the other two sets of data were obtained using an Astrodon Photometrics Sloan $i$ filter. The MORC24 mount has excellent free-running tracking, so we did not use a separate guide camera. Instead, minor telescope pointing corrections are made after each exposure by comparing the CCD pixel coordinates of the centroid of the target star to its initial position on the CCD. KELT-1b was held to within 3-4 pixels of the starting position on the CCD throughout each observing session. Since KELT-1b is separated from its nearest detectable neighbor in DSS2 imagery by $\sim 18^{\prime \prime}$, we were able to defocus the telescope to allow for longer exposures without the risk of blending from the neighbor star. An exposure time of $100 \mathrm{~s}$ was used for all observations, resulting in a $120 \mathrm{~s}$ cadence when combined with the $20 \mathrm{~s}$ CCD readout time.

We used AstroImageJ (K. Collins \& J. F. Kielkopf 2012, in preparation) to calibrate the image data. The algorithm includes bias subtraction, CCD nonlinearity correction, dark subtraction, and flat-field division. AstroImageJ was also used to perform aperture photometry using a circular aperture. An aperture size and an ensemble of comparison stars in the same field were chosen to minimize the scatter in the final light curves. AstroImageJ provides the option to use a standard fixed radius aperture or a variable radius aperture based on the measured FWHM of the target star in each image of the series. When a star is well separated from other stars, the variable aperture option tends to reduce photometric scatter under observing conditions that result in significant changes to the PSF during the observing session. The variable aperture produced optimal results for all four MORC24 KELT-1b light curves.

For the observations starting on UT 2011 December 2, cirrus clouds were present during the first half of the observations, and air mass ranged from 1.16 at the start of observations to 3.19 at the end. For the observations starting on UT 2011 December 4, skies were clear until clouds moved in about 30 minutes after ingress. The clouds cleared just prior to egress, however, sky transparency remained highly variable until about an hour after egress. Air mass ranged from 1.05 at the beginning of observations to 1.40 at the end. Although guiding was maintained through the cloud cover, data during that time have been removed. For the observations starting on UT 2011 December 31, skies were clear with minimal variations in transparency. Air mass ranged from 1.00 at the beginning of 


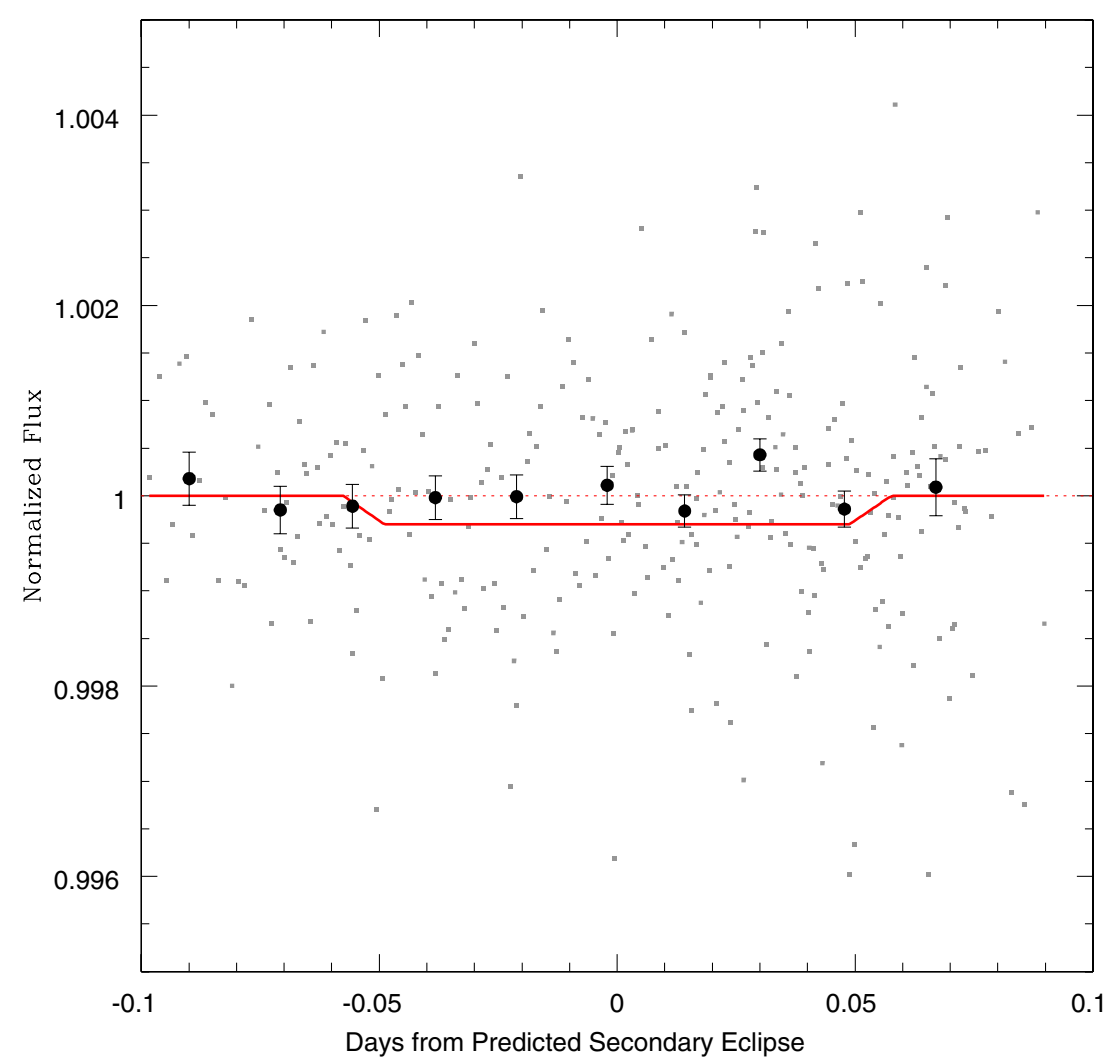

Figure 6. Gray points show the combined $i$ and Pan-STARRS- $Z$ relative photometry of KELT- 1 as a function of the predicted time of secondary eclipse of KELT- $1 \mathrm{~b}$ $\left(T_{S}\right)$, obtained from the observatories listed in Table 2. The data have been corrected for a linear trend with air mass and normalized by the zero point of the linear fit (see Section 5.4). The larger circles with error bars show the binned observations. Note that we do not fit to the binned data; these are shown for the purposes of illustration only. The over plotted example light curve is the secondary eclipse depth we would expect if KELT-1b had a geometric albedo of $A_{g}=0.1$ and instantaneously reradiated its incident stellar flux $\left(f^{\prime}=2 / 3\right)$. We would have detected this event with a confidence level of $\gtrsim 95 \%$.

(A color version of this figure is available in the online journal.)

observations to 2.17 at the end. For the observations on UT 2012 January 4, cirrus clouds were present during the second half of the observations, and air mass ranged from 1.03 at the start of observations to 1.96 at the end.

\subsubsection{Hereford Arizona Observatory (HAO)}

Data on the transit starting UT 2011 December 10 were obtained at the Hereford Arizona Observatory, HAO (observatory code G95 in the IAU Minor Planet Center). This is a private observatory in Southern Arizona consisting of a 14 inch Meade LX-200 GPS telescope equipped with an SBIG ST-10XME CCD, a focal reducer and a 10 position filter wheel with SDSS filters ugriz. The telescope and dome are operated via buried cables, permitting automation of observing sessions. Calibrations usually employ a master flat frame obtained during dusk prior to the observing session. The field of view $(27 \times 18 \mathrm{arcmin})$ is sufficient for the use of approximately two dozen stars as candidates for reference in a typical field. The observations reported here were obtained with the $i$ filter.

The data were reduced and a light curve was generated as follows. An artificial star was inserted in each image before photometry readings for the purpose of monitoring smooth extinction as well as extra extinction events caused by thin clouds, dew formation, and atmospheric seeing degradations that could swell the PSF beyond the photometry aperture circle. Photometry magnitude readings were made by the program MaxIm DL and imported to a spreadsheet, where several steps of manual reduction were completed. The first was to solve for an extinction model (including a temporal extinction trend)

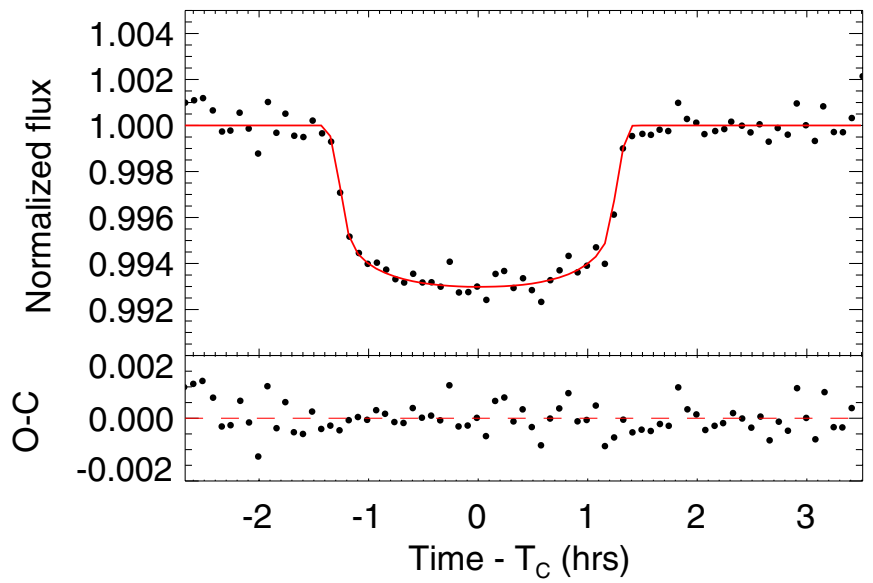

Figure 7. Top panel: the points show the six data sets displayed in Figure 5, combined and binned in five minute intervals. Since these data sets were taken with different filters and have different systematics, we do not use this combined light curve for analysis, but rather show it for the purposes of illustrating the overall quality and statistical constraining power of the ensemble of followup light curves. The solid curve shows the six transit models for each of the individual fits combined and binned in five minute intervals the same way as the data. Bottom panel: the residuals of the binned light curve from the binned model in the top panel.

(A color version of this figure is available in the online journal.)

based on the sum of all candidate reference star fluxes versus air mass. Second, subsets of reference stars were evaluated for suitability, by toggling individual stars "on and off" in order to 
determine the subset that minimize the rms scatter in the target star light curve.

Finally, the light curve for the target was fitted using a model for systematic effects and a transit signature. Systematics were represented by a temporal trend and air-mass curvature (AMC). The AMC is caused by the target star having a color that differs from the flux-weighted color of the reference stars. The transit parameters were depth, total duration, ingress/egress duration, and a parameter related to the stellar limb darkening. The solution was obtained by minimizing the $\chi^{2}$ of the fit. Outliers were identified using an objective rejection criterion based on deviations from the model solution. Finally, the light curve was corrected for extinction and systematic effects and scaled to the out-of-transit model flux.

\subsubsection{FLWO/KeplerCam}

Data on the transits on UT 2011 December 16 and 2012 January 7 were obtained with KeplerCam on the $1.2 \mathrm{~m}$ telescope at FLWO. KeplerCam has a single $4 \mathrm{~K} \times 4 \mathrm{~K}$ Fairchild CCD with a pixel scale of 0.366 arcsec pixel $^{-1}$, for a total FOV of $23.1 \times 23.1$ arcmin. A full transit was observed on UT 2011 December 16 with clear conditions. Observations were obtained in the SDSS $z$ filter with $30 \mathrm{~s}$ exposures. We also obtained a full transit on UT 2012 January 7 and observations were obtained with the SDSS $i$ filter with $15 \mathrm{~s}$ exposures. Clouds came in at the end of the transit and as a result there is some increased scatter in the out-of-transit baseline. The data were reduced using a light curve reduction pipeline outlined in Carter et al. (2011) which uses standard IDL techniques.

\subsubsection{Las Cumbres Observatory Global Telescope Network (LCOGT)}

Data on the secondary eclipse on UT 2011 December 30 were obtained with the $2.0 \mathrm{~m}$ Faulkes Telescope North (FTN) telescope, which is located on Haleakala on the island of Maui in Hawaii. The FTN telescope is part of the Las Cumbres Observatory Global Telescope Network. ${ }^{22}$ These observations were made using the $4 \mathrm{~K} \times 4 \mathrm{~K}$ Spectral camera (Fairchild Imaging CCD486 BI) in bin $2 \times 2$ mode for a faster readout, together with the PanSTARRS- $Z$ filter. As scintillation noise becomes significant ( $>1$ millimag) in exposures shorter than $\sim 30 \mathrm{~s}$ for telescopes of this aperture, the exposure time was kept to $60 \mathrm{~s}$ and the telescope defocused to avoid saturation of the target while ensuring sufficient signal-to-noise ratio in the comparison stars. These data were debiased, dark-subtracted, and flat fielded by the LCOGT offline pipeline (developed by the Astrophysics Research Institute at Liverpool John Moores) and aperture photometry was carried out using the stand-alone DAOPHOT II (Stetson 1987, 1990). Differential photometry was then computed using an ensemble of 15 comparison stars.

\subsection{Keck Adaptive Optics Imaging}

To further assess the multiplicity of KELT-1, we acquired adaptive optics images using NIRC2 (PI: Keith Matthews) at Keck on UT 2012 January 7. Our observations consist of dithered frames taken with the $K^{\prime}\left(\lambda_{c}=2.12 \mu \mathrm{m}\right)$ and $\mathrm{H}$ $\left(\lambda_{c}=1.65 \mu \mathrm{m}\right)$ filters. We used the narrow camera setting to provide fine spatial sampling of the stellar PSF. The total on-source integration time was $81 \mathrm{~s}$ in each bandpass.

Images were processed by replacing hot pixel values, flatfielding, and subtracting thermal background noise. No companions were identified in individual raw frames during the

\footnotetext{
22 http://lcogt.net
}

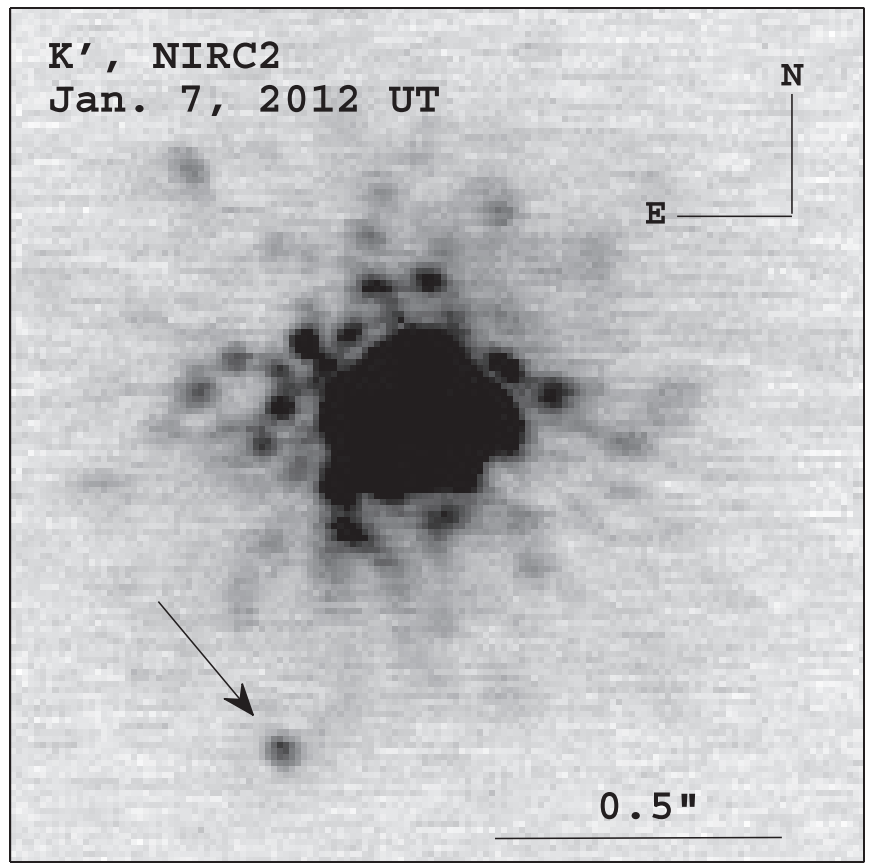

Figure 8. Keck AO image of KELT-1 taken with NIRC2 on UT 2012 January 7 in the $K^{\prime}$ filter. North is up and east is to the left. A $0^{\prime \prime} .5$ bar is shown for scale. A faint companion with $\Delta K^{\prime}=5.59 \pm 0.12$ located $\sim 558 \pm 1$ mas to the southeast is clearly visible.

observations; however, upon stacking the images we noticed a point source $(8 \sigma)$ to the southeast of KELT-1. Figure 8 shows the final processed $K^{\prime}$ image. Inspection of the companion location showed that its separation from the star does not change with wavelength, demonstrating that it is not a speckle. This object is too faint and close to the primary to be detected with seeing-limited images.

We performed aperture photometry to estimate the relative brightness of the candidate tertiary, finding $\Delta H=5.90 \pm 0.10$ and $\Delta K^{\prime}=5.59 \pm 0.12$. An $H-K^{\prime}=0.4 \pm 0.2$ color is consistent with spectral-types M1-L0 (Leggett et al. 2002; Kraus \& Hillenbrand 2007). If the candidate is bound to KELT-1 and thus at the same distance of $263 \pm 14$ pc and suffers the same extinction of $A_{V}=0.18 \pm 0.10$ (see Section 5.1), then we estimate its absolute $H$ magnitude to be $M_{H}=8.31 \pm 0.15$, corresponding to an M4-5 spectral type, consistent with its color (see, e.g., the compilation of Kirkpatrick et al. 2012).

We also measured an accurate position of the companion relative to the star by fitting a Gaussian model to each of the PSF cores. After correcting for distortion in the NIRC2 focal plane, ${ }^{23}$ and adopting a plate scale value of $9.963 \pm 0.006$ mas pixel $^{-1}$ and instrument orientation relative to the sky of $0.13 \pm 0.02$ (Ghez et al. 2008), we find a separation of $\rho=588 \pm 1$ mas and position angle P.A. $=157.4 \pm 0.2$ east of north. If it is bound to KELT-1, it has a projected physical separation of $\sim 154 \pm 8 \mathrm{AU}$, and a period of $\sim 1700$ years assuming a circular, face-on orbit.

We used the Galactic model from Dhital et al. (2010) to assess the probability that the companion is an unrelated star (i.e., a chance alignment). The model uses empirical number density distributions to simulate the surface density of stars along a given line of sight and thus determine probability of finding a star within a given angular separation from KELT-1.

$\overline{23}$ http://www2.keck.hawaii.edu/inst/nirc2/forReDoc/post_observing/dewarp/ 


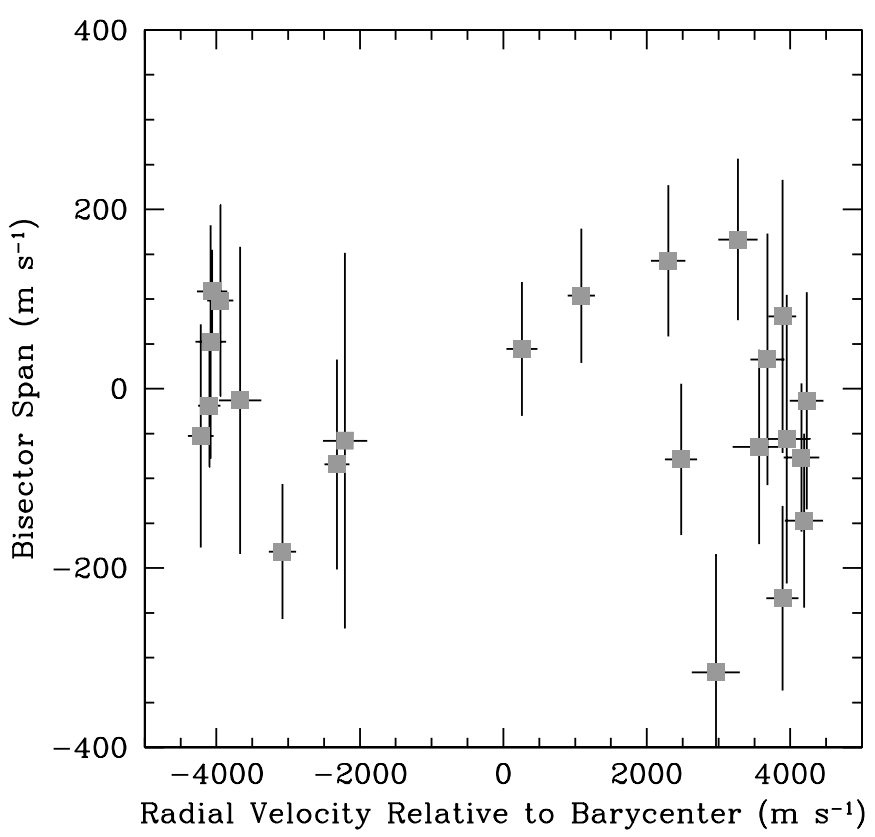

Figure 9. Bisector spans vs. the RV relative to the system barycenter, excluding observations taken on the night of the transit on UT 2012 January 7. There is no evidence of a significant correlation between the bisector and RV variations, and the rms of the bisector span variations is $\sim 30$ times smaller than the rms of the RV measurements.

We estimate an a priori probability of $\sim 0.05 \%$ of finding a star separated by $\lesssim 0^{\prime \prime} .59$ from KELT-1b. We therefore conclude that the companion is likely to be a bona fide, physically associated binary system. With a total proper motion of $\sim 20$ mas $\mathrm{yr}^{-1}$, it will be possible to definitively determine whether the candidate tertiary is physically associated with KELT-1 within 1 year.

We note that the companion is unresolved in our follow-up transit photometry, and thus in principle leads to a dilution of the transit signal and a bias in the parameters we infer from a fit to the photometry described in Section 5.2. However, the effect is negligible. As we discuss in the next section, we are confident that the primary is being eclipsed. Thus, the fractional effect on the transit depth is of the same order as the fractional contribution of the companion flux to the total flux. For the photometric bands where we have transit photometry, and assuming the companion is an M1 star or later, we estimate that it contributes $\lesssim 0.2 \%$ to the flux from the primary.

\section{EVIDENCE AGAINST A BLEND SCENARIO}

One of the many challenges of photometric surveys for transiting planets is the relatively high rate of astrophysical false positives, blended eclipsing stellar binary or triple systems that can mimic some of the observable signatures of transiting lowmass companions to single stars. In the case of the KELT-North survey, one generically expects a higher rate of false positives as compared to other wide-field transit surveys such as HATNet or SuperWASP, because of the poorer image quality arising from the comparatively smaller aperture, larger pixel scale, and wider FOV. For KELT-1 in particular, the extreme properties of the companion, relatively high $v \sin I_{*}$ of the primary, and the fact that the primary is somewhat evolved, are all reminiscent of false positives that have been reported in previous surveys, e.g., Mandushev et al. (2005).

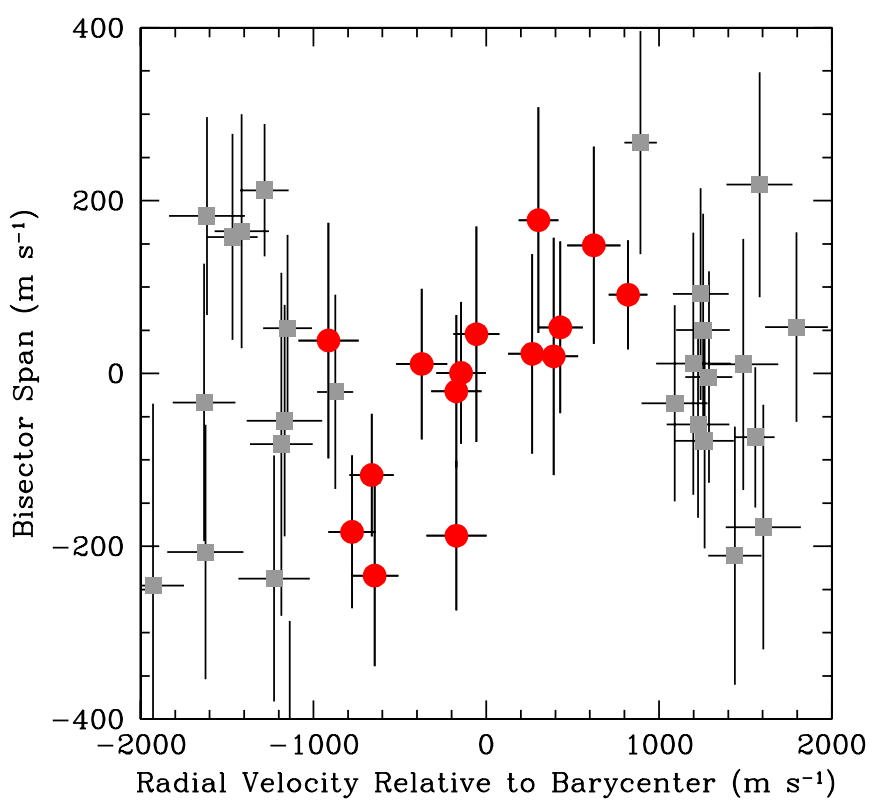

Figure 10. Bisector spans vs. the RV relative to the system barycenter for observations taken on the night of the transit on UT 2012 January 7. The circles are the subset of those data that were taken within 0.03 days of the center of the transit, roughly corresponding to the middle half of the full transit duration. Note that these data are strongly correlated with the RV variations due to the RM effect.

(A color version of this figure is available in the online journal.)

In the case of KELT-1b, however, we have a number of lines of evidence that strongly disfavor a blend scenario.

The most compelling arguments against blend scenarios arise from the spectra. First is the lack of strong evidence for bisector span variations. The lower panel of Figure 3 shows the bisector variations phased to the best-fit period of the companion as determined from the joint fit to the RV and photometry data described in Section 5.2. There is no evidence for bisector variations correlated with the orbital phase of the companion. The weighted rms of the bisector spans, excluding the data taken on UT 2012 January 7 , is $\sim 120 \mathrm{~m} \mathrm{~s}^{-1}$, only $\sim 30 \%$ larger than would be expected based on the native uncertainties, and a factor of $\sim 30$ times smaller than the rms of the RV measurements themselves. Figure 9 shows the bisector spans as a function of $\mathrm{RV}$ relative to the system barycenter. There is no strong correlation; the correlation coefficient is only -0.17 . In contrast, Figure 10 shows data taken on the night of UT 2012 January 7 , which covered the transit. For the subset of these data taken within 0.03 days of the transit center (approximately the middle half of the transit), there is a clear correlation between the RV and the bisector variations, with a correlation coefficient of 0.68 . This is expected since the anomalous RV shift from the RM effect is due to a distortion of the rotationally broadened stellar spectral lines as the planet progressively occults the light from different parts of the face of the star. Indeed, the second piece of evidence that the transit signatures are indeed due to a small companion occulting the primary star is the RM signal itself (Figure 4), which has an amplitude consistent with the apparent transit depth and spectroscopically determined $v \sin I_{*}$.

Third, photometric observations in several different filters (riz) are all consistent with the transit having nearly the same depth, and are well-modeled by transits of a dark companion across a star with the limb darkening consistent with its spectroscopically measured $T_{\text {eff }}$ and $\log g$ (see Section 5.2). 


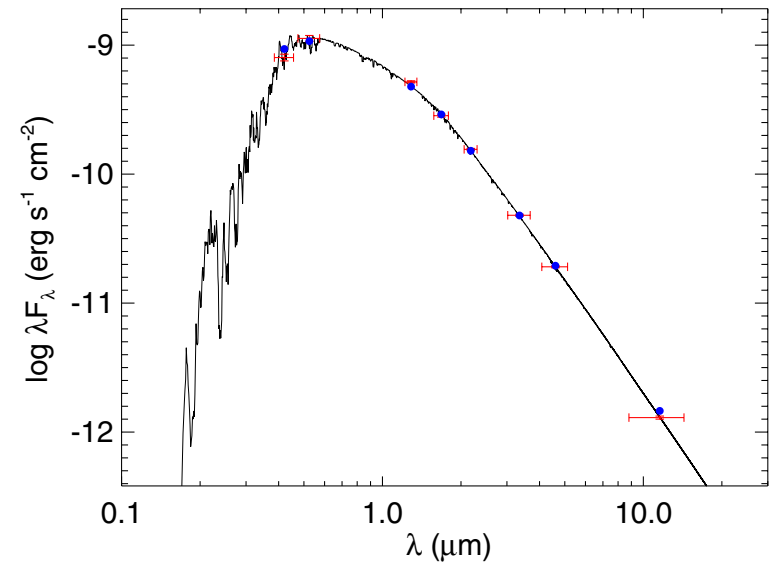

Figure 11. Error bars indicate measurements of the flux of KELT-1 host star in various optical and IR passbands. The vertical error bar indicates the photometric uncertainty, whereas the horizontal error bar indicates the effective width of the passband. The solid curve is the best-fit theoretical SED from the NextGen models of Hauschildt et al. (1999), assuming $T_{\text {eff }}, \log g$, and $[\mathrm{Fe} / \mathrm{H}]$ fixed at the values in Table 4 , with $A_{V}$ and $d$ allowed to vary. The dots are the predicted passband-integrated fluxes of the best-fit theoretical SED.

(A color version of this figure is available in the online journal.)

Fourth, photometric observations at the predicted time of superior conjunction reveal no evidence for a secondary eclipse at the $\lesssim 1$ mmag level. The third and fourth pieces of evidence tend to exclude or strongly disfavor blend scenarios in which the observed transits are due to diluted eclipses of a much fainter and redder eclipsing binary (e.g., O'Donovan et al. 2006).

Finally, our adaptive optics imaging does not reveal any sources further than $\sim 0$ '. 25 from the primary that could be both blended with it in seeing-limited images and cause transits at the observed depth of $\sim 1 \%$. The one source we do detect, the putative tertiary, has a flux ratio relative to the primary of only $\sim 0.5 \%$ in the near-IR, and is likely considerably fainter in the optical, and thus is too faint to explain the observed transits.

We did not perform any detailed modeling to determine the viability of specific blend scenarios. We defer here to Bakos et al. (2012), who argue that such analyses are generally unnecessary in situations in which there are no significant bisector variations, the transit ingress/egress durations are short compared to the total duration, and the RV variations phase with the predicted transit ephemeris.

We conclude that all of the available data are best explained as due to a Jupiter-sized, BD companion transiting a rapidly rotating mid-F star, with little or no evidence for significant contamination from blended sources. Under this assumption, we proceed in the following section to analyze these data in order to determine the physical properties of the KELT- 1 host star and its short-period, low-mass companion.

\section{CHARACTERIZATION OF THE STAR, COMPANION, AND ORBIT}

\subsection{Properties of the Host Star}

Table 3 lists various collected properties and measurements of the KELT-1 host star. Many of these have been culled from the literature, and the remainder are derived in this section. In summary, KELT-1 is a mildly evolved, solar-metallicity, mid-F star with an age of $\sim 1.5-2$ Gyr located at a distance of $\sim 260 \mathrm{pc}$, with kinematics consistent with membership in the thin disk.

We constructed an empirical spectral energy distribution (SED) of KELT-1 using optical fluxes in the $B_{T}$ and $V_{T}$

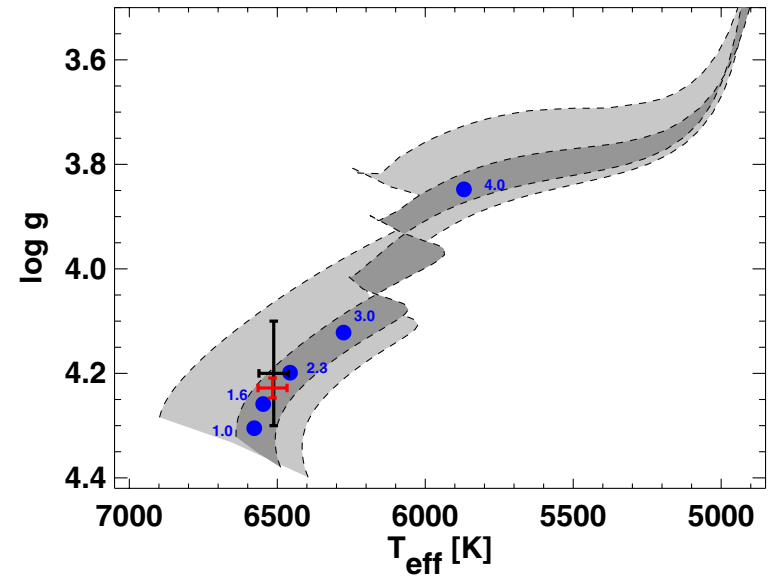

Figure 12. Theoretical H-R diagram based on Yonsei-Yale stellar evolution models (Demarque et al. 2004). The inner two sets of dashed tracks (shaded darked gray) represent the tracks for the extreme range of the $1 \sigma$ uncertainties on $M_{*}$ and $[\mathrm{Fe} / \mathrm{H}]$ for the host star, as inferred from the joint fit described in Section 5.2, specifically $M_{*}=1.335 \pm 0.063 M_{\odot}$ and $[\mathrm{Fe} / \mathrm{H}]=0.052 \pm 0.079$. The red cross shows the best-fit $T_{\text {eff }}=6516 \pm 49 \mathrm{~K}$ and $\log g=4.228_{-0.021}^{+0.014}$ from the final analysis. The black cross shows the inferred $T_{\text {eff }}$ and $\log g$ from the spectroscopic analysis alone. The blue dots represent the location of the star for various ages in Gyr. The host star is slightly evolved with a probable age of $\sim 2$ Gyr, although a similar analysis with a different stellar evolutionary model prefers a slightly younger age of $\sim 1.75$. The outer two sets of dashed tracks (shaded dark gray) show the range inferred using the spectroscopic constraints alone.

(A color version of this figure is available in the online journal.)

passbands from the Tycho-2 catalog (Høg et al. 2000), nearinfrared (IR) fluxes in the $J, H$, and $K s$ passbands from the 2MASS Point Source Catalog (Skrutskie et al. 2006; Cutri et al. 2003), and near- and mid-IR fluxes in three of the WISE passbands (Wright et al. 2010; Cutri et al. 2012). This SED is shown in Figure 11. We fit this SED to NextGen models from Hauschildt et al. (1999) by fixing the values of $T_{\text {eff }}, \log g$, and $[\mathrm{Fe} / \mathrm{H}]$ inferred from the global fit to the light curve and $\mathrm{RV}$ data as described in Section 5.2 and listed in Table 4, and then finding the values of the visual extinction $A_{V}$ and distance $d$ that minimizes $\chi^{2}$. We find $A_{V}=0.18 \pm 0.10$ and $d=263 \pm 14 \mathrm{pc}$, with a $\chi^{2}=10.5$ for 6 degrees of freedom, indicating a reasonable fit $\left(P\left(>\chi^{2}\right) \sim 10 \%\right)$. We also performed a fit to the SED without priors, finding $T_{\text {eff }}=6500 \pm 400 \mathrm{~K}, A_{V}=0.20 \pm 0.15, \log g=4.25 \pm 0.75$, and $[\mathrm{Fe} / \mathrm{H}]=-0.5 \pm 0.5$, consistent with the constrained fit. There is no evidence for an IR excess.

We note that the quoted statistical uncertainties on $A_{V}$ and $d$ are likely to be underestimated, because we have not accounted for the uncertainties in values of $T_{\text {eff }}, \log g$, and $[\mathrm{Fe} / \mathrm{H}]$ used to derive the model SED. Furthermore, it is likely that alternate model atmospheres would predict somewhat different SEDs and thus values of the extinction and distance.

In Figure 12, we plot the predicted evolutionary track of KELT-1 on a theoretical H-R diagram ( $\log g$ versus $T_{\text {eff }}$ ), from the Yonsei-Yale stellar models (Demarque et al. 2004). Here, again we have used the values of $M_{*}$ and $[\mathrm{Fe} / \mathrm{H}]$ derived from the global fit (Section 5.2 and Table 4). We also show evolutionary tracks for masses corresponding to the $\pm 1 \sigma$ extrema in the estimated uncertainty. In order to estimate the age of the KELT-1 system, we compare these tracks to the values of $T_{\text {eff }}$ and $\log g$ and associated uncertainties as determined from the global fit. These intersect the evolutionary track for a fairly narrow range of ages near $\sim 2$ Gyr. The agreement between the prediction from 
the evolutionary track at this age and the inferred temperature and surface gravity for KELT-1 is remarkably good, but perhaps not entirely surprising. The values of $T_{\text {eff }}, \log g,[\mathrm{Fe} / \mathrm{H}]$, and $M_{*}$ were all determined in the global fit to the light curve and RV data in Section 5.2, which uses the empirical relations between both $M_{*}$ and $R_{*}$ and $\left(T_{\text {eff }}, \log g,[\mathrm{Fe} / \mathrm{H}]\right)$ inferred by Torres et al. (2010) as priors on the fit, in order to break the well-known degeneracy between $M_{*}$ and $R_{*}$ for single-lined spectroscopic eclipsing systems. These empirical relations are known to reproduce the constraints between these parameters imposed by the physics of stellar evolution quite well (see, e.g., Section 8 in Torres et al. 2010).

Based on its $T_{\text {eff }}$ and $J-K$ and the empirical table of spectral type versus color and $T_{\text {eff }}$ for main sequence from Kenyon \& Hartmann (1995), we infer the spectral type of KELT-1 to be F5 with an uncertainty of roughly \pm 1 spectral type.

We determined the Galactic $U, V, W$ space velocities of the KELT-1 system using the proper motion of $\left(\mu_{\alpha}, \mu_{\delta}\right)=$ $(-10.1 \pm 0.7,-9.4 \pm 0.7)$ mas $^{-1} r^{-1}$ from the NOMAD catalog (Zacharias et al. 2004), the distance of $d=263 \pm 14$ pc from our SED fit described above, and the barycentric RV of the system as determined from the TRES observations (Section 3.3) of $\gamma_{\mathrm{abs}}=-14.2 \pm 0.2 \mathrm{~km} \mathrm{~s}^{-1}$. We used a modification of the IDL routine GAL_UVW, which is itself based on the method of Johnson \& Soderblom (1987). We adopt the correction for the Sun's motion with respect to the Local Standard of Rest from Coşkunoğlu et al. (2011), and choose a right-handed coordinate system such that positive $U$ is toward the Galactic Center. We find $(U, V, W)=(19.9 \pm 1.1,-9.6 \pm 0.5,-2.6 \pm 0.9) \mathrm{km} \mathrm{s}^{-1}$, consistent with membership in the thin disk (Bensby et al. 2003). We note also that the distance of KELT-1 from the Galactic plane is $\sim 80 \mathrm{pc}$.

Finally, we use the solar evolutionary models of Guenther et al. (1992), updated with input physics from van Saders \& Pinsonneault (2012), to gain some insight into the detailed structure of the host star. Fixing the mass and metallicity at the values determined from the global fit (Section 5.2 and Table 4), we evolved the model forward until the model $\log g$ and $T_{\text {eff }}$ approximately matched the values inferred for KELT- 1 . We found that an age of $\sim 1.75 \mathrm{Gyr}$ best matched the available constraints, and thus this model prefers a somewhat younger age than the Yale-Yonsei model of Demarque et al. (2004, Figure 12). We therefore decided to adopt an age of $1.75 \pm$ $0.25 \mathrm{Gyr}$, consistent with both estimates. For this range of ages, the models of Guenther et al. (1992) predict a radius of the base of the convective zone of $R_{c z}=1.31 \pm 0.03 R_{\odot}$ and a very small mass for the convective zone of $M_{c z}=[2.8 \pm 0.4] \times 10^{-4} M_{\odot}$, as expected given the effective temperature of $T_{\text {eff }} \sim 6500 \mathrm{~K}$. In addition, the moment of inertia for the star and convective zone are $C_{*}=[1.18 \pm 0.04] \times 10^{54} \mathrm{~g} \mathrm{~cm}^{2}$ and $C_{c z}=$ $[3.2 \pm 0.7] \times 10^{51} \mathrm{~g} \mathrm{~cm}^{2}$, respectively. We can also write the moment of inertia of the star as $C_{*}=\alpha_{*} M_{*} R_{*}^{2}$ with $\alpha_{*}=0.042$ (Guenther et al. 1992). We will use these to estimate the angular momenta of the star, companion, and orbit in Section 6.2.

\subsection{System Properties Derived from a Joint Fit}

It is well known that a joint fit to high-quality RVs and transit photometry of a transiting planet system allows one to determine the mass and radius of the star and planet, as well as the semimajor axis of the orbit, in principle to very high precision, up to a perfect one-parameter degeneracy (Seager \& Mallén-Ornelas 2003). This degeneracy arises because the duration, depth, and shape of the transit, when combined with the eccentricity, longitude of periastron, and period of the planet from RV data, allow one to precisely estimate the density of the primary star $\rho_{*}$, but not $M_{*}$ or $R_{*}$ separately. Breaking this $M_{*}-R_{*}$ degeneracy generally requires imposing some external constraint, such as a theoretical mass-radius relation (Cody \& Sasselov 2002; Seager \& Mallén-Ornelas 2003) or constraints from theoretical isochrones (e.g., Bakos et al. 2012). In principle, a measurement of $\log g$ from a high-resolution spectrum can be used to break the degeneracy, but in practice these measurements are generally not competitive with the constraint on $\rho_{*}$ and often have systematic uncertainties that are comparable to the statistical uncertainties.

We fitted the RV and transit data using a modified version of the IDL fitting package EXOFAST (Eastman et al. 2012). The approach of EXOFAST to breaking the $M_{*}-R_{*}$ degeneracy is similar to the method described in, e.g., Anderson et al. (2012), but with significant differences. We will review it briefly here, but direct the reader to Eastman et al. (2012) for more details. We fitted the RV and transit data simultaneously with a transit photometric model (Mandel \& Agol 2002) and a standard Keplerian RV model using a modified MCMC method (described in more detail below). In addition to the standard fitting parameters, we also included $T_{\text {eff }}, \log g$, and $[\mathrm{Fe} / \mathrm{H}]$ as proposal parameters. We then included priors on the measured values of $T_{\text {eff }}, \log g$, and $[\mathrm{Fe} / \mathrm{H}]$ as determined from analysis of the TRES spectra and given in Section 3.3. In addition, we included separate priors on both $M_{*}$ and $R_{*}$, each of which are based on the empirical relations between $M_{*}$ and $R_{*}$ and $\left(T_{\text {eff }}, \log g,[\mathrm{Fe} / \mathrm{H}]\right)$ determined by Torres et al. (2010). These priors essentially break the $M_{*}-R_{*}$ degeneracy, as they provide similar constraints as isochrones, i.e., they encode the mapping between the $M_{*},[\mathrm{Fe} / \mathrm{H}]$, and age of a star to its $T_{\text {eff }}$ and $\log g$ as dictated by stellar physics.

We fitted the six transits, Doppler RV, stellar parameters, and RM effect simultaneously using EXOFAST, which employs a Differential Evolution Markov Chain Monte Carlo (DE-MC) method (ter Braak 2006). We converted all times to the BJD $\mathrm{BDB}_{\mathrm{T}}$ standard (Eastman et al. 2010), and then at each step in the Markov Chain, we converted them to the target's barycentric coordinate system (ignoring relativistic effects). Note that the final times were converted back to $\mathrm{BJD}_{\mathrm{TDB}}$ for ease of use. This transformation accurately and transparently handles the light travel time difference between the RVs and the transits.

First, we fitted the Doppler RV data independently to a simple Keplerian model, ignoring the RM data taken on UT 2012 January 7. At this stage, we did not include any priors on the stellar parameters, as they do not affect the RV-only fit. We scaled the uncertainties such that the probability $P\left(>\chi^{2}\right)$ of finding a value of $\chi^{2}$ equal to or larger than the value we measured was 0.5 , in order to ensure that the resulting parameter uncertainties were roughly accurate. For a uniform prior in eccentricity, we found the orbit is consistent with circular, with a $3 \sigma$ upper limit of $e<0.04$. Nevertheless, in order to provide conservative estimates for the fit parameters, we allowed for a non-zero eccentricity in our final fit. However, to test the effect of this assumption, we repeated the final fit forcing $e=0$. We also investigated the possibility of a slope in the RVs, but found it to be consistent with zero, so we did not include this in the final fit.

Next, we fitted each of the four transits individually, including a zero point, $F_{0, i}$ and air-mass detrending variable, $C_{0, i}$ for each of the $i$ transits. The air-mass detrending coefficient was significant $(>1 \sigma)$ for all but one transit, so for consistency, we 
included it for all. After finding the best fit with AMOEBA (Nelder \& Mead 1965), we scaled the errors for each transit such that $P\left(>\chi^{2}\right)=0.5$. At this stage, we included the priors on the stellar parameters as described above.

Next, we performed a combined fit to all the data, including a prior on the projected stellar rotation velocity $\left(v \sin I_{*}=\right.$ $55.2 \pm 2.0 \mathrm{~km} \mathrm{~s}^{-1}$ ) from the spectra, ${ }^{24}$ and a prior on the period from the KELT-North discovery light curve $(P=$ $1.217513 \pm 0.000015$ days). Because it is usually systematics in the RV data that vary over long timescales (due to a combination of instrumental drift and stellar jitter) that ultimately set the error floor to the RVs, we expect the uncertainties of densely packed observations to be smaller than the rms of all observations, but with a systematic offset relative to the rest of the orbit. Therefore, we fitted a separate zero point during the RM run, and also scaled the errors on the RVs during transit to force $P\left(>\chi^{2}\right)=0.5$ for those subsets of points. $P\left(>\chi^{2}\right)$ depends on the number of degrees of freedom, but it is not obvious how many degrees of freedom there are in the RM run-technically, the entire orbit and the transit affect the $\chi^{2}$ of the RM (13 parameters), but the freedom of the RM measurements to influence most of those parameters is very limited, when fit simultaneously with the transits. Indeed, even $v \sin I_{*}$ is constrained more by the spectroscopic prior than the RM in this case, which means there are only two parameters (the projected spin-orbit alignment, $\lambda$, and the zero point, $\gamma$ ) that are truly free. To be conservative, we subtracted another degree of freedom to encompass all the other parameters on which the RM data have a slight influence, before scaling the errors.

The RM data were modeled using the Ohta et al. (2005) analytic approximation with linear limb darkening. ${ }^{25}$ At each step in the Markov Chain, we interpolated the linear limbdarkening tables of Claret \& Bloemen (2011) based on the chain's value for $\log g, T_{\text {eff }}$, and $[\mathrm{Fe} / \mathrm{H}]$ to derive the linear limbdarkening coefficient, $u$. We assumed the $V$-band parameters to approximate the bandpass of TRES, though we repeated the exercise in the $B$ band with no appreciable difference in any of the final parameters. Note that we do not fit for the limb-darkening parameters, as the data are not sufficient to constrain them directly. The uncertainties in all the limbdarkening parameters provided in Table 5 arise solely from the scatter in $\log g, T_{\text {eff }}$, and $[\mathrm{Fe} / \mathrm{H}]$. We assume no error in the interpolation of the limb-darkening tables.

In order to search for Transit Timing Variations (TTVs), during the combined fit, we fitted a new time of transit, $T_{C, i}$ for each of the $i$ transits. Therefore, the constraint on $T_{C}$ and $P$ (quoted in Tables 5 and 4, respectively) comes from the prior imposed from the KELT-North light curve and the RV data, not the follow-up light curves. Using these times to constrain the

\footnotetext{
24 The prior on $v \sin I_{*}$ improves the determination of the spin-orbit alignment angle $\lambda$ (Gaudi \& Winn 2006). We also performed a fit without this prior, finding results that were roughly consistent with, although less precise than, those with the prior.

25 We note that the Ohta et al. (2005) formula is known to be a poor approximation for the RM signal when $v \sin I_{*}$ is large, as is the case for KELT-1. Hirano et al. (2011) provide a formula for the RM effect that is more appropriate for large $v \sin I_{*}$. We have compared the RM signal calculated using the Hirano et al. (2011) formula to that calculated using the Ohta et al. (2005) formula for the best-fit parameters of the KELT-1 system. We find maximum differences of $\sim 50 \mathrm{~m} \mathrm{~s}^{-1}$, confined to two relatively short phases near ingress and egress. Given the large (100-200 $\left.\mathrm{m} \mathrm{s}^{-1}\right) \mathrm{RV}$ uncertainties for the RM measurements, we estimate the difference in $\chi^{2}$ between these two models is $\sim 1$. Thus, using the more correct formula of Hirano et al. (2011) would have a negligible effect on our inferred RM parameters or their uncertainties.
}

period during the fit would artificially reduce any observed TTV signal. A separate constraint on $T_{C}$ and $P$ follows from fitting a linear ephemeris to the transit times, as discussed in Section 5.3. It is the result from this fit that we quote as our final adopted ephemeris.

The results from this global fit are summarized in Tables 4 and 5 . We also show the results for the physical parameters assuming $e=0$ in Table 4; the differences between the fixed and free eccentricity fits are always smaller than their uncertainties, and generally negligible for most of these parameters. The values of $T_{\text {eff }}, \log g$, and $[\mathrm{Fe} / \mathrm{H}]$ we infer from the global fit are in agreement with the values measured directly from the TRES spectra to within the uncertainties. Since the spectroscopic values were used as priors in the global fit, this generally indicates that there is no tension between the value of $\rho_{*}$ inferred from the light curve and RV data, and the spectroscopic values. The median value and uncertainty for $T_{\text {eff }}$ and $[\mathrm{Fe} / \mathrm{H}]$ are nearly unaffected by the global fit. On the other hand, the uncertainty in $\log g$ from the global fit is a factor of $\sim 5$ smaller than the uncertainty from the spectroscopic measurement. This is not surprising, since the constraint on $\rho_{*}$ from the RV and light curve data provides a strong constraint on $\log g$ via the relations of Torres et al. (2010).

We also present in Table 4 our estimates of the median values and uncertainties for a number of derived physical parameters of the system that may be of interest, including the planet equilibrium temperature assuming zero albedo and perfect redistribution $T_{\text {eq }}$, the average amount of stellar flux at the orbit of the companion $\langle F\rangle$, and the Safronov number $\Theta=\left(a / R_{p}\right)\left(M_{P} / M_{*}\right)$ (e.g., Hansen \& Barman 2007). In addition, in Table 5 we quote our estimates of various fit parameters and intermediate derived quantities for the Keplerian RV fit, the transits, and the secondary eclipse.

We note that the final uncertainties we derive for $M_{*}, R_{*}$, $\log g$, and $\rho_{*}$ are relatively small, $\sim 3 \%-6 \%$. These uncertainties are of the same order as those found for other transiting planet systems using methods similar to the one used here (e.g., Anderson et al. 2012). Specifically, these methods derive physical parameters from a fit to the light curve and RV data, which simultaneously impose empirically determined relations between $M_{*}$ and $R_{*}$ and $\left(T_{\text {eff }}, \log g,[\mathrm{Fe} / \mathrm{H}]\right)$, which are ultimately derived from Torres et al. (2010). These constraints help to break the $M_{*}-R_{*}$ degeneracy pointed out by Seager \& Mallén-Ornelas (2003) and discussed above. These Torres et al. (2010) relations, including the scatter about these relations, in concert with the constraint on $\rho_{*}$ from the transit light curves, largely determine the final uncertainty on $M_{*}$ and $R_{*}$ (and thus $M_{P}$ and $R_{P}$ ). In particular, we find that our spectroscopic measurement of $\log g$ provides a much weaker constraint. Given that our results rely so heavily on the Torres et al. (2010) relations, it is worthwhile to ask to what extent our parameters and uncertainties might be affected should these relations be systematically in error. First, as already noted, these empirical relations are known to agree well with stellar isochrones in general (Torres et al. 2010), and for KELT-1 in particular (Figure 12). Second, analyses using stellar isochrones rather than empirical relations produce similar uncertainties on $M_{*}$ and $R_{*}$ (e.g., Bakos et al. 2011), suggesting that the small uncertainties we derive are not a by-product of our specific methodology. Finally, Southworth (2009) demonstrated that the results of the analysis of 14 transiting systems with several different sets of isochrones generally agree to within $\sim 2 \%-5 \%$. This suggests that the systematic errors caused by uncertainties 


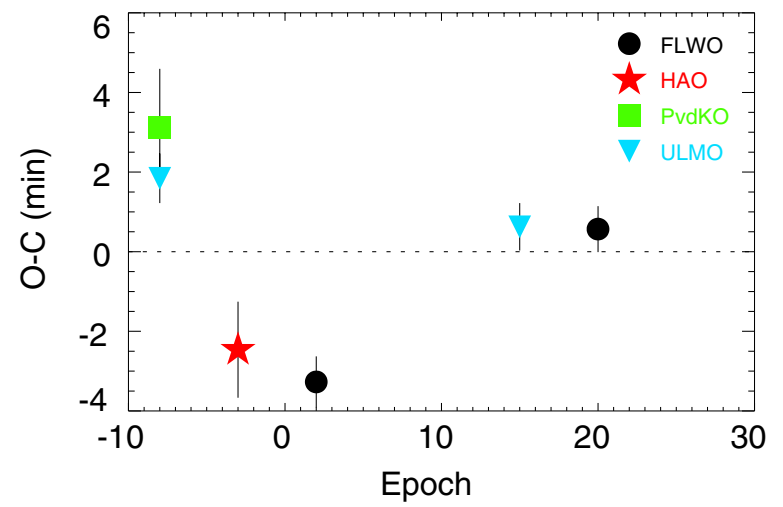

Figure 13. Residuals of the transit times from the best-fit (linear) ephemeris. The transit times are given in Table 6.

(A color version of this figure is available in the online journal.)

in the isochrones (and so the Torres et al. 2010 relations) are of the same order as the statistical uncertainties we infer. We therefore conclude that our results are likely accurate, with systematic errors at the level of our statistical uncertainties (a few to several percent).

\subsection{System Ephemeris and Transit Timing Variations}

Table 6 lists the measured transit times for each of the six modeled transits, and Figure 13 shows the residuals of these times from a fit to a linear ephemeris. The best fit has

$$
\begin{aligned}
T_{C}\left(\mathrm{BJD}_{\mathrm{TDB}}\right) & =2455909.29280 \pm 0.00023 \\
P & =1.217501 \pm 0.000018,
\end{aligned}
$$

which is consistent with the ephemeris derived from the KELTNorth light curve alone. The $\chi^{2}=45.5$ for the linear fit with 4 degrees of freedom is formally quite poor. This is mostly driven by one nominally significant $(5 \sigma)$ outlier, specifically for the transit observed on UT 2011 December 16 from FLWO. We note that the faint companion to KELT-1, if indeed bound, is too distant to explain such large TTVs.

We have taken great care to ensure the accuracy of our clocks and our conversion, and the fact that the residuals from different observatories roughly follow the same trend in time suggests that a catastrophic error in the observatory clock cannot be blamed. Since we fit the trend with air mass simultaneously with the transit, the potentially large covariance between it and the transit time should be accurately reflected in the quoted uncertainties (Eastman et al. 2012). Nevertheless, our MCMC analysis does not adequately take into account the effect of systematic uncertainties, and in particular we do not account for correlated uncertainties (Pont et al. 2006b; Carter \& Winn 2009), which could skew the transit time of a given event substantially. And, given the results from Kepler which suggest the rarity of such TTVs (Steffen et al. 2012), we are reluctant to overinterpret this result. Nevertheless, this is an interesting target for future follow-up.

\subsection{Secondary Eclipse Limits}

We observed the predicted secondary eclipses of KELT-1b assuming $e=0$ on UT 2011 December 2, 2011 December 30, and 2012 January 4 . In none of the three were we able to detect a secondary eclipse. The observations on 2011 December 2 and on 2012 January 4 were taken from the ULMO Observatory in $i$. On both nights we were able to observe through the predicted ingress and egress of the potential secondary. The two $i$-band light curves have a combined 236 data points, and show an rms scatter of $1.56 \mathrm{mmag}$. The observations on 2011 December 30 were taken with the FTN telescope in PanSTARRS-Z. In this case, we were only able to begin observations half way through the predicted secondary eclipse. This $Z$-band light curve has 72 points, and an rms scatter of $0.75 \mathrm{mmag}$.

We used the system parameters derived from the joint fit (Section 5.2) to fit our three observations. Since we did not detect a secondary eclipse, we used these fits to explore the combination of heat redistribution efficiency and Bond albedo $A_{B}$ that would give rise to a secondary eclipse depth that is inconsistent with our data. To do so, we calculated the secondary eclipse depths we would expect for a range of redistribution efficiencies and albedos, and then fit a secondary eclipse model with the predicted depth to all three of our observations simultaneously.

In calculating the expected secondary eclipse depths, we made the assumption that both the star and the planet were blackbodies. We also assumed that the planet was a gray Lambert sphere, so the geometric albedo $A_{g}=(2 / 3) A_{B}$, and the spherical albedo is constant as a function of wavelength. Following Seager (2010), we parameterized the heat redistribution efficiency as $f^{\prime}$, which is $1 / 4$ in the case of uniform redistribution, and $2 / 3$ in the case of no redistribution. We note that in between these two extremes, $f^{\prime}$ is not easily related to the amount of heat redistribution, i.e., $f^{\prime}=0.45$ does not imply that half of the incident stellar energy is redistributed around the planet.

To test these expected secondary eclipse depths against our observations, we fit simple trapezoidal eclipse curves with the expected depths to all three data sets simultaneously. Under our assumptions, the depth, timing, shape, and duration of the secondary eclipse are all determined by the parameters derived from the global fit and our specified values of $A_{B}$ and $f^{\prime}$. We then fit this model to our data, allowing for a normalization and a linear trend in the flux with time. We used the $\Delta \chi^{2}$ between the best-fit eclipse model and the best constant fit, which itself was allowed a free slope and offset, to evaluate the detectability of each of the secondary eclipse depths. We used the $\chi^{2}$ distribution to transform these $\Delta \chi^{2}$ values into detection probabilities. Figure 6 shows an example light curve against a median binned version of our data. This particular curve is the secondary eclipse we would expect if KELT-1b had $A_{B}=0.1$, and instantaneously re-radiated its incident stellar flux, i.e., $f^{\prime}=2 / 3$. We would have detected this event with more than $95 \%$ confidence.

Figure 14 shows the results of our exploration of the heat redistribution versus Bond albedo parameter space. Contours where the eclipse depths are detectable at the $68 \%, 90 \%$, and $95 \%$ confidence level are indicated. The particular shapes of the contours on this plot come from the competing effects of reflection and blackbody emission from KELT-1b on the depth of the secondary eclipse. Along the very bottom of the figure the Bond albedo is zero, and thus there is only thermal emission. We see the strong change in eclipse depth as the amount of heat redistribution decreases, thus causing the temperature and eclipse depth for KELT-1b to increase. Along the top of the figure, where the Bond albedo is 0.75 , the reflected starlight dominates the blackbody emission such that changing the redistribution efficiency has little effect on the eclipse depth.

Slightly more than half of the allowed parameter space in Figure 14 would have caused secondary eclipses detectable in 


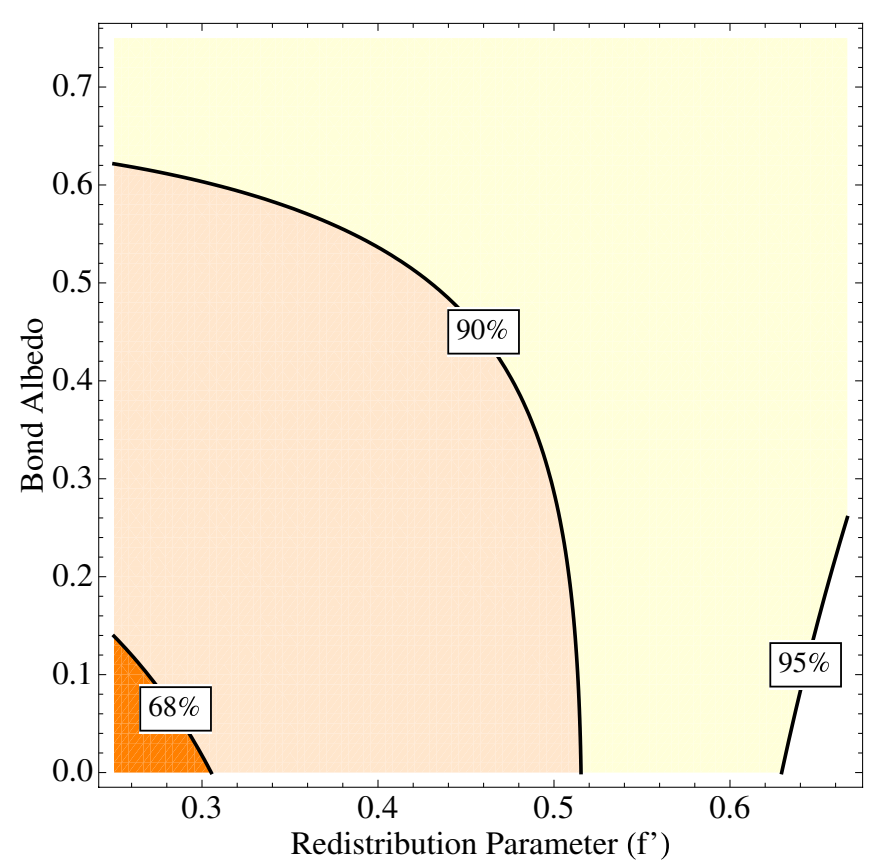

Figure 14. Values of the heat redistribution parameter $f^{\prime}$ and Bond albedo $A_{B}$ that are excluded at a given confidence level based on the data taken during the secondary eclipse shown in Figure 6. The $f^{\prime}$ parameter describes the efficiency of heat redistribution and is $1 / 4$ in the case of uniform redistribution, and $2 / 3$ in the case of no redistribution. In between these two extremes, $f^{\prime}$ is not easily related to the amount of heat redistribution. Contours where the eclipse depths are detectable at the $68 \%, 90 \%$, and $95 \%$ confidence level (left to right) are indicated.

(A color version of this figure is available in the online journal.)

our data at greater than $90 \%$ confidence, while almost all would have been detected at more than $68 \%$ confidence. Since we did not see a secondary eclipse in our observations, we conclude that either KELT-1b has a non-zero albedo, or it must redistribute some heat from the day side, or both. Formally, the scenario that is most consistent with our data is that KELT-1b has both a low Bond albedo and is very efficient at redistributing heat away from its day side, however we are reluctant to draw any strong conclusions based on these data.

\section{DISCUSSION}

From our global fit to the light curves and RVs, we find that KELT-1b is a low-mass companion with a measured mass $M_{P}=27.38 \pm 0.93 M_{\text {Jup }}$ and radius $R_{P}=1.116_{-0.029}^{+0.038} R_{\text {Jup }}$. It is on a circular orbit with a semimajor axis of $a=0.02472 \pm$ 0.00039 AU. The host KELT-1 is a mildly evolved mid-F star with a mass $M_{*}=1.335 \pm 0.063 M_{\odot}$, radius $R_{*}=$ $1.471_{-0.035}^{+0.045} R_{\odot}$, effective temperature $T_{\text {eff }}=6516 \pm 49 \mathrm{~K}$, and a likely age of $\sim 1.5-2$ Gyr. Because of its small semimajor axis and hot host, KELT-1b receives a large stellar insolation flux of $\langle F\rangle=7.83_{-0.34}^{+0.45} \times 10^{9} \mathrm{erg} \mathrm{s}^{-1} \mathrm{~cm}^{-2}$, implying a high equilibrium temperature of $T_{\mathrm{eq}}=2423_{-27}^{+34} \mathrm{~K}$ assuming zero albedo and perfect redistribution. Both the surface gravity and density of KELT-1b are substantially higher than that of its host star, and higher than we would expect for a stellar object. We find that the orbit normal of KELT-1b is well aligned with the projected rotation axis of its host star, with a projected alignment angle of $\lambda=2 \pm 16 \mathrm{deg}$.

Even among the large and diverse menagerie of known transiting exoplanets and low-mass companions, KELT-1b is unique. First, it is one of only seven unambiguous objects with the mass range $\sim 13-80 M_{\text {Jup }}$ that are known to transit stars. Among these, it has the shortest period and orbits the brightest host star $(V=10.7)$. In addition, there is potentially a stellar M dwarf companion to the primary. For all these reasons, KELT-1b is likely to be a very interesting object for further study, and we expect it will provide a benchmark system to test theories of the emplacement and evolution of short period companions, as well the physics of tidal dissipation and irradiated atmospheres of substellar objects. We will discuss some of these ideas briefly.

\subsection{Brown Dwarf or Supermassive Planet? KELT-1b and the Brown Dwarf Desert}

Is KELT- $1 \mathrm{~b}$ a BD or is it a suppermassive planet? By IAU convention, BDs are defined to have masses between the deuterium burning limit of $\sim 13 M_{\text {Jup }}$ (Spiegel et al. 2011) and the hydrogen burning limit of $\sim 80 M_{\text {Jup }}$ (e.g., Burrows et al. 1997). Less massive objects are defined to be planets, whereas more massive objects are stars. By this definition, KELT-1b is a low-mass BD. However, it is interesting to ask whether or not KELT-1b could have plausibly formed in a protoplanetary disk, and therefore might be more appropriately considered a "supermassive planet" (Schneider et al. 2011). More generally, it is interesting to consider what KELT-1b and systems like it may tell us about the formation mechanisms of close companions with masses in the range of 10-100 $M_{\text {Jup }}$.

One of the first results to emerge from precision Doppler searches for exoplanets is the existence of a BD desert, an apparent paucity BD companions to FGK stars with periods less than a few years, relative to the frequency of stellar companions in the same range of periods (Marcy \& Butler 2000). Subsequent studies uncovered planetary companions to such stars in this range of periods in abundance (Cumming et al. 2008), indicating that the BD desert is a local nadir in the mass function of companions to FGK stars. The simplest interpretation is that this is the gap between the largest objects that can form in a protoplanetary disk, and the smallest objects that can directly collapse or fragment to form a self-gravitating object in the vicinity of a more massive protostar. Therefore, the location of KELT-1b with respect to the minimum of the BD mass function might plausibly provide a clue to its origin.

\subsubsection{Comparison Sample of Transiting Exoplanets, Brown Dwarfs, and Low-mass Stellar Companions}

In order to place the parameters of KELT-1b in context, we construct a sample of transiting exoplanets, BDs, and lowmass stellar companions to main-sequence stars. We focus only on transiting objects, which have the advantage that both the mass and radius of the companions are precisely known. ${ }^{26}$ We collect the transiting exoplanet systems from the Exoplanet Data Explorer (exoplanets.org; Wright et al. 2011), discarding systems for which the planet mass is not measured. We supplement this list with known transiting BDs (Deleuil et al. 2008; Johnson et al. 2011; Bouchy et al. 2011a, 2011b; Anderson et al. 2011). We do not include the system discovered

\footnotetext{
26 In contrast, for companions detected only via RVs, only the minimum mass is known. Of course, one can make an estimate of the posterior probability distribution of the true mass given a measured minimum mass by adopting a prior for the distribution of inclinations (e.g., Lee et al. 2011). However, this procedure can be particularly misleading in the case of BDs: if BDs are indeed very rare, then objects with minimum mass in the BD desert are more likely to be stellar companions seen at low inclination. Anecdotally, in those cases where constraints on the inclinations can be made, companions with minimum mass near the middle of the BD desert often do turn out to be stars (e.g., Sahlmann et al. 2011; Fleming et al. 2012).
} 
by Irwin et al. (2010), because a radius measurement for the BD was not possible. We also do not include 2M0535 - 05 (Stassun et al. 2007) because it is a young, double BD system. We add several transiting low-mass stars near the hydrogen burning limit (Pont et al. 2005, 2006a; Beatty et al. 2007). We adopt the mass of XO-3b from the discovery paper (Johns-Krull et al. 2008), which is $M_{P}=13.1 \pm 0.4 M_{\mathrm{Jup}}$, which straddles the deuterium burning limit (Spiegel et al. 2011). However, later estimates revised the mass significantly lower to $M_{P}=11.8 \pm 0.6$ (Winn et al. 2008). We will therefore categorize XO-3b as an exoplanet.

The disadvantage of using samples culled from transit surveys is that the sample size is much smaller, and transit surveys have large and generally unquantified selection biases (e.g., Gaudi et al. 2005; Fressin et al. 2009), particularly groundbased transit surveys. We emphasize that such biases are almost certainly present in the sample we construct. We have therefore made no effort to be complete. The comparisons we make based on this sample should not be considered definitive, but rather suggestive.

Figure 15 places KELT-1b among the demographics of known transiting companions to main-sequence stars, focusing on massive exoplanets, BDs, and low-mass stars with short periods of $\lesssim 30$ days. KELT-1b has the tenth shortest period of any transiting exoplanet or BD known. It has the sixth shortest period among giant $\left(M_{P} \gtrsim 0.1 M_{\text {Jup }}\right)$ planets, with only WASP-19b, WASP-43b, WASP-18b, WASP-12b, OGLE-TR-56b, and HAT-P-23b having shorter periods. KELT-1b is more massive by a factor $\sim 3$ than the most massive of these, WASP-18b (Hellier et al. 2009). KELT-1b has a significantly shorter period than any of the previously known transiting BDs, by a factor of $\gtrsim 3$. KELT-1b therefore appears to be located in a heretofore relatively unpopulated region of the $M_{P}-P$ parameter space for transiting companions.

Although the KELT-1 system is relatively unique, it is worth asking if there are any other known systems that bear some resemblance to it. The $M_{P} \sin i \simeq 18 M_{\mathrm{Jup}}, P \simeq 1.3$ day RVdiscovered companion to the M dwarf HD 41004B (Zucker et al. 2003) has similar minimum mass and orbit as KELT-1b; however, the host star is obviously quite different. Considering the host star properties as well, perhaps the closest analogs are WASP-18b (Hellier et al. 2009), WASP-33b (Collier Cameron et al. 2010), and KOI-13b (Mazeh et al. 2012; Mislis \& Hodgkin 2012). All three of these systems consist of relatively massive $\left(M_{p} \gtrsim 3 M_{\mathrm{Jup}}\right)$ planets in short $(\lesssim 2$ day) orbits around hot $\left(T_{\text {eff }} \gtrsim 6500 \mathrm{~K}\right)$ stars.

The mass of KELT-1b $\left(\sim 27 M_{\mathrm{Jup}}\right)$ is close to the most arid part of the BD desert, estimated to be at a mass of $31_{-18}^{+25} M_{\mathrm{Jup}}$ according to Grether \& Lineweaver (2006). Thus, under the assumption that the BD desert reflects the difficulty of forming objects with this mass close to the parent star under any formation scenario, KELT-1b may provide an interesting case to test these various models. For disk scenarios, gravitational instability can likely form such massive objects, but likely only at much larger distances (Rafikov 2005; Dodson-Robinson et al. 2009; Kratter et al. 2010). The maximum mass possible from core accretion is poorly understood, but may be as large as $\sim 40 M_{\text {Jup }}$ (Mordasini et al. 2009). The possibility of significant migration of KELT-1b from its birth location to its present position must also be considered, particularly given the existence of a possible stellar companion to KELT-1 (Section 3.5). This possibility complicates the interpretation of the formation of KELT-1b significantly. For example, it has been suggested that $\mathrm{BD}$ companions are more common at larger
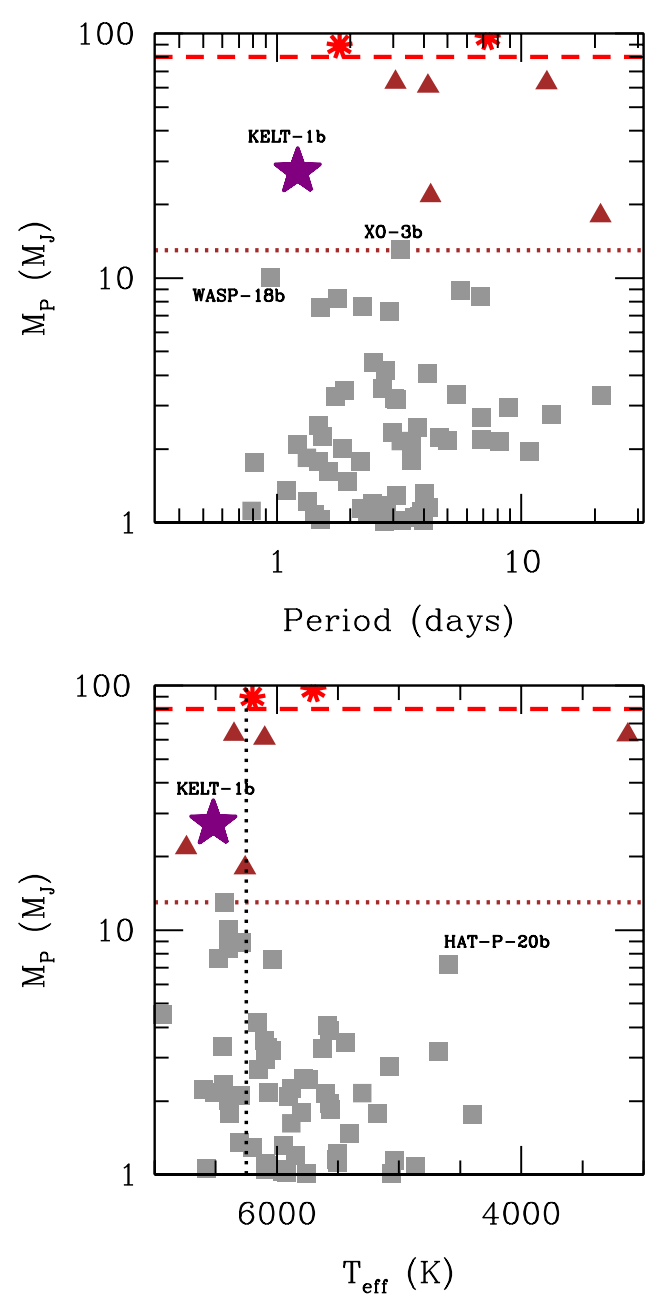

Figure 15. Top panel: mass vs. period for the known transiting companions to main-sequence stars with companion masses in the range 1-100 $M_{J}$. An estimate of the deuterium burning limit (Spiegel et al. 2011) is shown as the horizontal dotted line, while the hydrogen burning limit is shown as the horizontal dashed line. Brown dwarfs are shown as triangles, exoplanets as squares, and low-mass stars as asterisks. KELT-1b is shown as the large star. It is the shortest period transiting brown dwarf currently known. Bottom panel: mass vs. host star effective temperature $T_{\text {eff }}$ for the sample of transiting companions shown in the top panel. As suggested by Bouchy et al. (2011a), there is some evidence that massive $\left(M_{P} \gtrsim 5 M_{\text {Jup }}\right)$ companions are preferentially found around hot $\left(T_{\text {eff }} \gtrsim 6000 \mathrm{~K}\right)$ stars, and KELT-1b follows this possible trend. The vertical line shows the division between hot and cool stars of $T_{\text {eff }}=6250 \mathrm{~K}$ suggested by Winn et al. (2010). Note that we exclude the BD companion to NLTT 41135 (Irwin et al. 2010), and the double BD transit system 2M0535 05 (Stassun et al. 2007) in this and subsequent plots.

(A color version of this figure is available in the online journal.)

separations (Metchev \& Hillenbrand 2009); thus KELT-1b may have formed by collapse or fragmentation at a large separation, and subsequently migrated to its current position via the Kozai-Lidov mechanism (Kozai 1962; Lidov 1962).

One clue to the origin of KELT-1b and the BD desert may be found by studying the frequency of close BD companions to stars as a function of the stellar mass or temperature. Figure 15 shows the mass of known transiting short period companions as a function of the effective temperature of the host stars. As pointed out by Bouchy et al. (2011a), companions with $M_{P} \gtrsim 5 M_{\text {Jup }}$ appear to be preferentially found around hot stars with $T_{\text {eff }} \gtrsim$ $6000 \mathrm{~K}$, and KELT-1b follows this trend. Although these hot stars are somewhat more massive, the most dramatic difference between stars hotter and cooler than $6000 \mathrm{~K}$ is the depth of their 


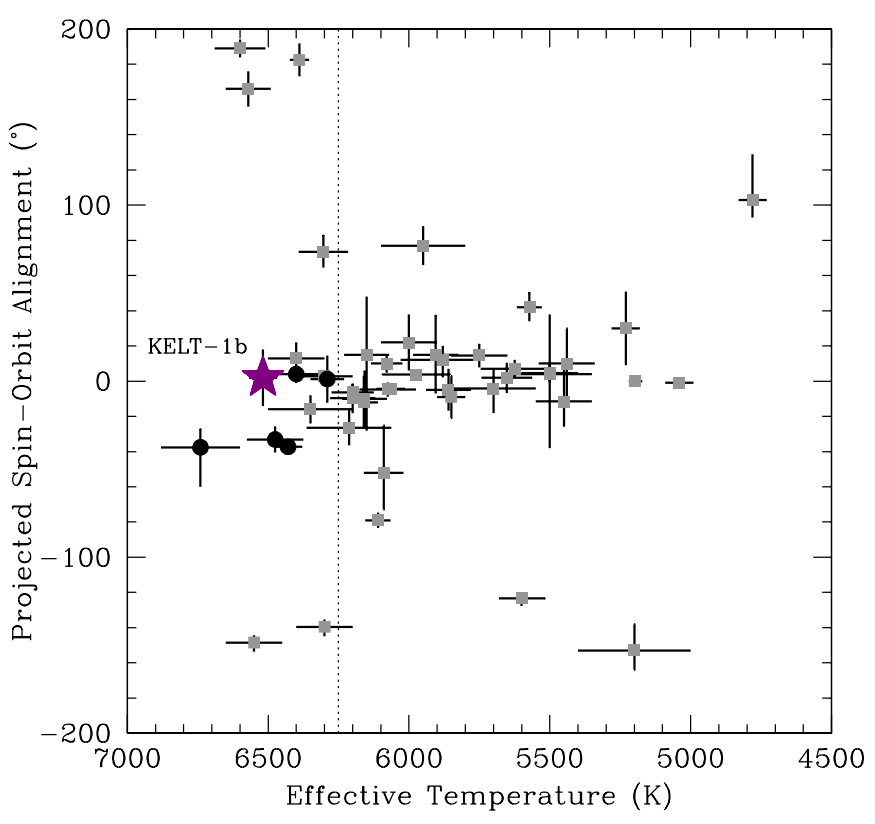

Figure 16. Projected spin-orbit alignment angle $\lambda$ for transiting planets as measured by the RM effect vs. the effective temperature of the host star, following Winn et al. (2010). The gray squares show exoplanets with mass $M_{P}<5 M_{\mathrm{Jup}}$, whereas the black circles show those with $M_{P}>5 M_{\mathrm{Jup}}$. KELT-1b, shown with a star, is the first transiting brown dwarf with an RM measurement. Its orbit normal is consistent with being aligned with the projected host star spin axis. The dotted vertical line shows the suggested dividing line between hot and cool stars by Winn et al. (2010).

(A color version of this figure is available in the online journal.)

convection zones. This led Bouchy et al. (2011a) to suggest that tides may play an important role in shaping the frequency and distribution of massive exoplanet and BD companions to old stars. Some evidence for this has been reported by Winn et al. (2010), who argue that hot $\left(T_{\text {eff }} \geqslant 6250 \mathrm{~K}\right)$ stars with close companions preferentially have high obliquities, suggesting that if the emplacement mechanisms are similar for all stars, tidal forces must later serve to preferentially bring cool host stars into alignment. Figure 16 shows the distribution of spin-orbit alignments for transiting planets versus the host star effective temperature. KELT-1b falls in the group of hot stars with small obliquities. Interestingly the other massive $\gtrsim 5 M_{\text {Jup }}$ planets are also located in this group.

We discuss the possible formation and evolutionary history of KELT-1b, and the likely role of tides in this history, in more detail below. We remain agnostic about the classification of KELT-1b as a BD or supermassive planet.

\subsection{Tides, Synchronization, and Kozai Emplacement}

Given the relatively large mass and short orbital period of KELT-1b, it seems probable that tides have strongly influenced the past evolution of the system and may continue to be affecting its evolution. The literature on the influence of tides on exoplanet systems is vast (see, e.g., Rasio et al. 1996; Ogilvie \& Lin 2004; Jackson et al. 2008; Leconte et al. 2010; Matsumura et al. 2010; Hansen 2010, for but a few examples), and there appears to be little consensus on the correct treatment of even the most basic physics of tidal dissipation, with the primary uncertainties related to where, how, and on what timescale the tidal energy is dissipated.

While we are interested in evaluating the importance of tides on the evolution of the orbit of KELT-1b and the spin of KELT-1, delving into the rich but difficult subject of tides is beyond the scope of this paper. We therefore take a somewhat heuristic approach. Specifically, we construct a few dimensionless quantities that likely incorporate the primary physical properties of binary systems that determine the scale of tidal evolution, but do not depend on the uncertain physics of energy dissipation. Specifically, we define,

$$
\begin{gathered}
\mathcal{T}_{a} \equiv \frac{M_{*}}{M_{P}}\left(\frac{a}{R_{*}}\right)^{5}, \quad \text { and } \\
\mathcal{T}_{\omega_{*}} \equiv\left(\frac{M_{*}}{M_{P}}\right)^{2}\left(\frac{a}{R_{*}}\right)^{3} .
\end{gathered}
$$

For some classes of theories of tidal dissipation and under some assumptions, $\mathcal{T}_{a}$ is proportional to the $e$-folding timescale for decay of the orbit, and $\mathcal{T}_{\omega_{*}}$ is proportional to the timescale for synchronization of the spin of the star with the companion orbital period. It is worthwhile to note that for transiting planet systems the combinations of parameters $M_{P} / M_{*}$ and $a / R_{*}$ are generally much better determined than the individual parameters. In particular, the ratio of the mass of the planet to that of the star is closely related to the RV semi-amplitude $K$, whereas $a / R_{*}$ is closely related to the ratio of the duration of the transit to the period (Winn 2010). Figure 17 shows $\mathcal{T}_{a}$ and $\mathcal{T}_{\omega_{*}}$ as a function of orbital period for the sample of transiting exoplanets, BDs, and low-mass stars discussed previously. KELT-1b has shorter timescales than nearly the entire sample of systems, with the exception of a few of the low-mass stars. We therefore expect tidal effects to be quite important in this system.

As a specific example, under the constant time lag model (Hut 1981; Matsumura et al. 2010), and assuming dissipation in the star, zero eccentricity, zero stellar obliquity, and a slowly rotating star, the characteristic timescale for orbital decay due to tides is $\tau_{\text {decay }} \equiv a /|\dot{a}|=(12 \pi)^{-1} Q_{*}^{\prime} \mathcal{T}_{a} P$, where $Q_{*}^{\prime}$ is related to the dimensionless tidal quality factor. For KELT-1b, $\mathcal{T}_{a} \sim 3 \times 10^{4}$, and so $\tau_{\text {decay }} \sim 0.3$ Gyr for $Q_{*}^{\prime}=10^{8}$, clearly much shorter than the age of the system. Similarly, the timescale for spinning up the star by the companion is $\tau_{\text {synch }} \equiv \omega_{*} /\left|\dot{\omega}_{*}\right| \propto Q_{*}^{\prime} \mathcal{T}_{\omega_{*}} P$ (Matsumura et al. 2010) and so is also expected to be short compared to the age of the system.

Given the expected short synchronization timescale and the fact that the expected timescale for tidal decay is shorter than the age of the system, it is interesting to ask whether or not the system has achieved full synchronization, thus ensuring the stability of KELT-1b. The measured projected rotation velocity of the star is $v \sin I_{*}=56 \pm 2 \mathrm{~km} \mathrm{~s}^{-1}$, which, given the inferred stellar radius, corresponds to a rotation period of $P_{*}=2 \pi R_{*} \sin I_{*} / v \sin I_{*}=[1.329 \pm 0.060] \sin I_{*}$ days, which differs from the orbital period of KELT-1b by $\sim 2 \sigma$ for $I_{*}=90^{\circ}$. This is suggestive that the system is indeed synchronized. The small discrepancy could either be due to a slightly underestimated uncertainty on $v \sin I_{*}$ or the host could be moderately inclined by $I_{*} \sim\left[66_{-5}^{+8}\right]^{\circ}$. However, one might expect the obliquity of the star to be realigned on roughly the same timescale as the synchronization of its spin (Matsumura et al. 2010). The stellar inclination can also be constrained by the precise shape of the transit light curve: lower inclinations imply higher rotation velocities, and thus increased oblateness and gravity brightening (Barnes 2009). Ultimately, the inclination is limited to $I_{*} \gtrsim 10^{\circ}$ in order to avoid break up.

We can also ask, given the known system parameters, if the system is theoretically expected to be able to achieve a stable 

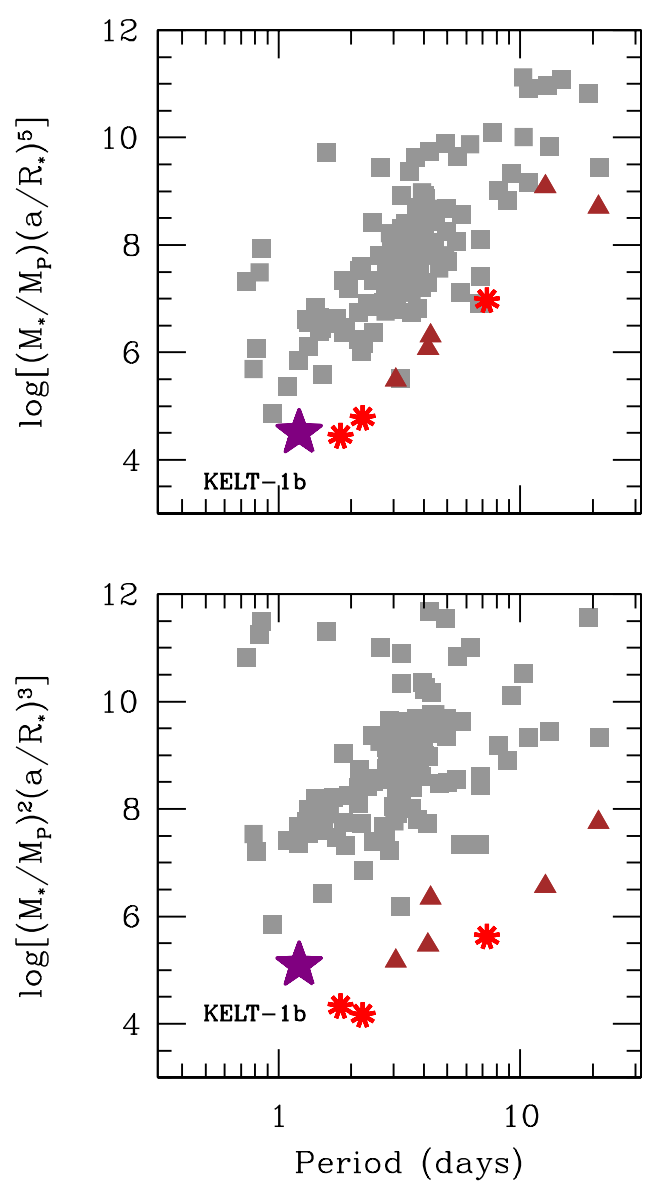

Figure 17. Dimensionless combinations of physical parameters that quantify the relative timescale for orbital tidal decay (top panel) and stellar spin-orbit synchronization (bottom panel) for different binary systems, as a function of the orbital period of the system. See Section 6.2 for an explanation and assumptions. Brown dwarfs are shown as triangles, exoplanets as squares, and low-mass stars as asterisks. KELT-1b is shown as the large star. Among known transiting exoplanets and brown dwarfs, it has the shortest characteristic timescale for orbital decay and synchronization.

(A color version of this figure is available in the online journal.)

synchronous state. A system is "Darwin stable" (Darwin 1879; Hut 1980) if its total angular momentum,

$$
L_{\mathrm{tot}}=L_{\mathrm{orb}}+L_{\omega, *}+L_{\omega, P},
$$

is more than the critical angular momentum of

$$
L_{\text {crit }} \equiv 4\left[\frac{G^{2}}{27} \frac{M_{*}^{3} M_{P}^{3}}{M_{*}+M_{P}}\left(C_{*}+C_{P}\right)\right]^{1 / 4},
$$

where $L_{\text {orb }}$ is the orbital angular momentum, $L_{\omega_{*}}$ is the spin angular momentum of the star, $L_{\omega, P}$ is the spin angular momentum of the planet, and $C_{*}=\alpha_{*} M_{*} R_{*}^{2}$ and $C_{P}=\alpha_{P} M_{P} R_{P}^{2}$ are the moments of inertia of the star and planet, respectively (Matsumura et al. 2010). Since $C_{P} / C_{*} \sim\left(M_{P} / M_{*}\right)\left(R_{P} / R_{*}\right)^{2} \sim$ $10^{-3}$, the contribution from the planet spin to the total angular momentum is negligible. We find $L_{\text {tot }} / L_{\text {crit }}=1.03 \pm 0.03$, consistent with the critical value for stability. In addition, we find $\left(L_{\omega, *}+L_{\omega, P}\right) / L_{\text {orb }}=0.15 \pm 0.01$, which is smaller than the maximum value of $1 / 3$ required for a stable equilibrium (Hut 1980). Curiously, if we assume the star is already tidally synchronized, we instead infer $\left(L_{\omega, *}+L_{\omega, P}\right) / L_{\text {orb }}=$ $0.17 \pm 0.01$, i.e., remarkably close to exactly one-half the critical value of $1 / 3$.
Two additional pieces of information potentially provide clues to the evolutionary history of this system: the detection of a possible tertiary (Section 3.5; Figure 8) and the measurement of the RM effect (Figure 4), demonstrating that KELT-1 has small projected obliquity. If the nearby companion to KELT-1 is indeed bound, it could provide a way of emplacing KELT-1b in a small orbit via the Kozai-Lidov mechanism (Kozai 1962; Lidov 1962). If KELT-1b were originally formed much further from its host star, and on an orbit that was significantly misaligned with that of the putative tertiary, then its orbit might subsequently be driven to high eccentricity via secular perturbations from the tertiary (Holman et al. 1997; Lithwick \& Naoz 2011; Katz et al. 2011). If it reached sufficiently high eccentricity such that tidal effects became important at periastron, the orbit would be subsequently circularized at a relatively short period (Fabrycky \& Tremaine 2007; Wu et al. 2007; Socrates et al. 2012). Nominally, one might expect the orbit of KELT-1b to be then left with a relatively large obliquity (Naoz et al. 2011). The measured projected obliquity is $\lesssim 16 \mathrm{deg}$, implying that either the current true obliquity is small or the star is significantly inclined (i.e., $I_{*} \sim 0$ ). However, if the star is significantly inclined, then the system cannot be synchronized. Perhaps a more likely alternative is that, after emplacement by the tertiary and circularization of the orbit, the system continued to evolve under tidal forces, with KELT-1b migrating inward to its current orbit while damping the obliquity of KELT-1 and synchronizing its spin period. Clearly, detailed simulations are needed to establish whether or not this scenario has any basis in physical reality.

\subsection{Comparison to Theoretical Models of Brown Dwarfs}

Transiting BDs provide one of the only ways to test and calibrate models of BD structure and evolution, which are used to interpret observations of the hundreds of free floating BDs for which no direct measurement of mass and radius is possible. Given that only five transiting BDs with radius measurements were previously known, KELT-1b potentially provides another important test of these models. Figure 18 shows the mass-radius relation for the known transiting companions to main-sequence stars with companion masses in the range 10-100 $M_{J}$. Being close to the minimum in the BD desert, the mass of KELT-1b begins to fill in the dearth of know systems between $\sim 20$ and $60 M_{\text {Jup }}$. Furthermore, the formal uncertainty in its radius is only $\sim 3 \%$, thereby allowing for a stringent test of models. In contrast, the two transiting BDs with similar masses, CoRoT-3b (Deleuil et al. 2008) and KOI-423b (Bouchy et al. 2011a), have much larger radius uncertainties, presumably due to the relative faintness of the host stars.

Evolutionary models for isolated BDs generally predict that young $(\sim 0.5 \mathrm{Gyr})$ objects in the mass range $10-100 M_{\text {Jup }}$ should have radii of $\sim R_{\text {Jup }}$ (see the models of Baraffe et al. 2003 in Figure 18, and also models by Burrows et al. 2011). As these objects cool, however, their radii decrease, particularly for masses between 50 and $80 M_{\text {Jup }}$. After $\sim 1 \mathrm{Gyr}$, all isolated objects with mass between 20 and $80 M_{\text {Jup }}$ are predicted to have radii $<R_{\text {Jup }}$. The radius we measure for KELT-1b is $R_{P}=$ $1.116_{-0.029}^{+0.038} R_{\mathrm{Jup}}$, which, at a mass of $M_{P}=27.38 \pm 0.93 M_{\mathrm{J}}$, is $\sim 6 \sigma$ and $\sim 8 \sigma$ larger than the radius predicted by Baraffe et al. (2003) for ages of $1 \mathrm{Gyr}$ and $5 \mathrm{Gyr}$, respectively. KELT-1b is strongly irradiated, which in principle can delay its cooling and contraction. However, Bouchy et al. (2011b) predict that the effect of insolation is small for BDs in this mass range, although their models were for a much more 


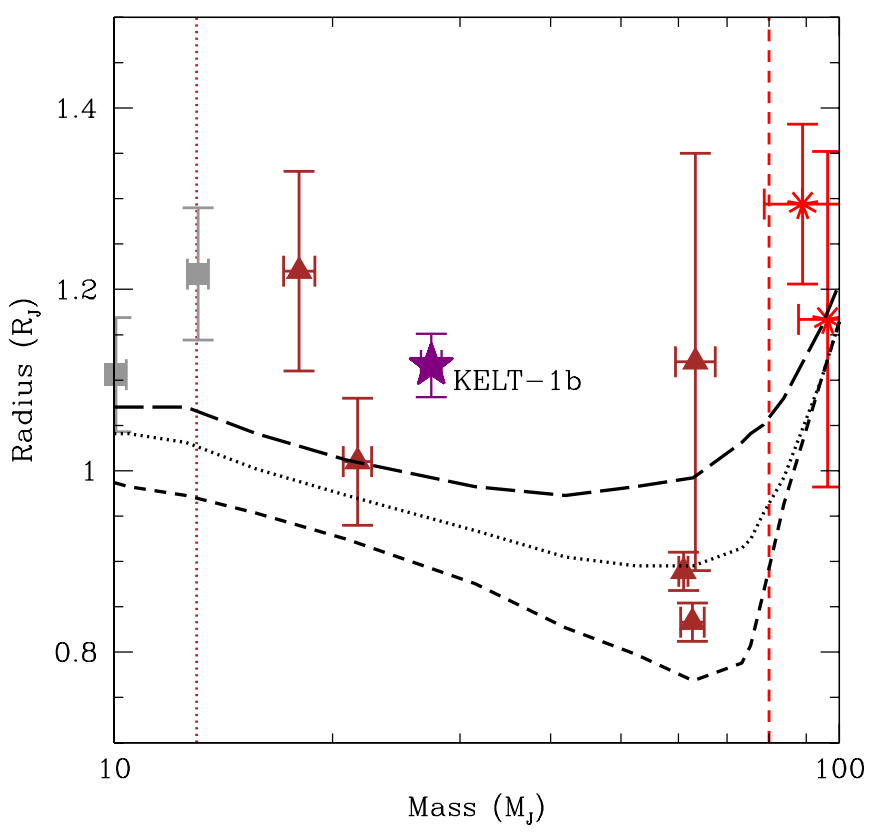

Figure 18. Radius vs. mass for the known transiting companions to mainsequence stars with companion masses in the range 10-100 $M_{J}$ that have measured radii. An estimate of the deuterium burning limit (Spiegel et al. 2011) is shown as the vertical dotted line, and the hydrogen burning limit is shown as the vertical dashed line. Brown dwarfs are shown as triangles, exoplanets as squares, and low-mass stars as asterisks. KELT-1b is shown as the large star. Predicted radii as a function of mass for isolated objects from the isochrones of Baraffe et al. (2003) are shown for an age of 5 Gyr (dashed), 1 Gyr (dotted), and 0.5 Gyr (long dashed); the true age of the KELT-1 system is almost certainly between 1 and 5 Gyr. Although stellar insolation is likely to increase the radii at fixed mass, Bouchy et al. (2011b) predict that the effect is small. KELT-1b therefore has an anomalously large radius.

(A color version of this figure is available in the online journal.)

modest insolation corresponding to an equilibrium temperature of $1800 \mathrm{~K}$ (versus $\sim 2400 \mathrm{~K}$ for KELT-1b). Therefore, given the estimated 1.5-2 Gyr age of the system, KELT-1b is likely to be significantly inflated relative to predictions.

Using the benchmark double transiting BD 2M0535-05, Gómez Maqueo Chew et al. (2009) explore models in which brown dwarfs have large spots, which reduce the flux from their surface, thereby decreasing their effective temperatures and increasing their radii relative to those without spots (see also Bouchy et al. 2011b). They find that these can lead to significantly inflated radii, but only for large spot filling factors of $\sim 50 \%$ and for relatively young $(\sim 0.5 \mathrm{Gyr})$ systems. However, a detailed spectroscopic analysis of that system by Mohanty et al. (2010) and Mohanty \& Stassun (2012) shows that surface spots cannot be present with such a large filling factor, and thus favor global structural effects such as strong internal magnetic fields (e.g., Mullan \& MacDonald 2010). Many other mechanisms have been invoked to explain the inflated radii of some giant exoplanets (see Fortney \& Nettelmann 2010 for a review); however, it is not clear which, if any, of the many mechanisms that have proposed may also be applied to inflated brown dwarfs.

We would be remiss if we did not question whether we were erroneously inferring a large radius for the planet. In the past, such situations have arisen when there is a discrepancy between the constraint on the stellar density from the light curve and the constraint on the stellar surface gravity of the star from spectroscopy (e.g., Johns-Krull et al. 2008; Winn et al. 2008).

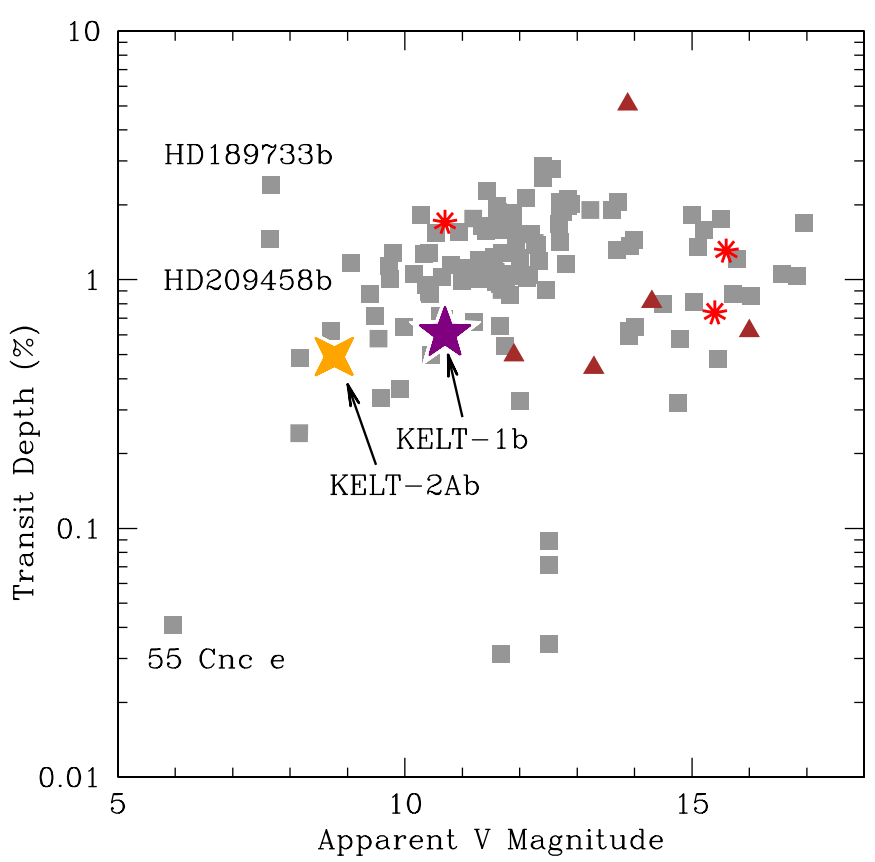

Figure 19. Transit depth assuming no limb darkening, i.e., $\left(R_{P} / R_{*}\right)^{2}$, as a function of the apparent $V$ magnitude of the host star for a sample of transiting systems. Brown dwarfs are shown as triangles, exoplanets as squares, and lowmass stars as asterisks. KELT-1b is shown as the large star. All else being equal, objects in the top left provide the best targets for follow-up. KELT-1b has a similar transit depth as the other known transiting brown dwarfs, but is significantly brighter. Also labeled are some other benchmark systems. KELT-2b $\left(M_{P} \sim 1.5 M_{\text {Jup }}\right)$ is shown as a large cross (Beatty et al. 2012).

(A color version of this figure is available in the online journal.)

In our case, we find no such tension. The parameters of the star inferred from the spectroscopic data alone are in nearly perfect agreement with the results from the global analysis of the light curve, RV data, and spectroscopic constraints. We note that the effect of allowing a non-zero eccentricity also has a negligible effect on the inferred planetary radius. Finally, we reiterate that the faint companion detected in $\mathrm{AO}$ imaging (Section 3.5), which is unresolved in our follow-up photometry, has a negligible effect on our global fit and inferred parameters. Therefore, we believe our estimate of $R_{P}$ is likely robust.

We conclude by noting that there is a need for predictions of the radii of brown dwarfs for a range of ages and stellar insolations, and it would be worthwhile to explore whether or not the inflation mechanisms that have been invented to explain anomalously large giant planets might work for much more massive and dense objects as well.

\subsection{Prospects for Follow-up}

Figure 19 compares the transit depth and apparent visual magnitude of the KELT-1 system $(\delta \sim 0.6 \%, V=10.7)$ to the sample of transiting systems collected in Section 6.1.1 with available $V$ magnitudes. KELT-1 is not particularly bright compared to the bulk of the known transiting exoplanet hosts. However, it is significantly brighter than the hosts of all known transiting brown dwarfs; the next brightest is WASP-30 (Anderson et al. 2011), which is $\sim 1.2$ mag fainter. On the other hand, the depth of the KELT-1b transit is similar to that of the other known brown dwarfs.

The prospects for follow-up of KELT-1b are exciting, not only because of the brightness of the host, but also because 
of the extreme nature of the system parameters, in particular the relatively short orbital period, relatively large stellar radius, and relatively large amount of stellar irradiation received by the planet. Following Mazeh \& Faigler (2010) and Faigler \& Mazeh (2011), we can estimate the amplitudes of ellipsoidal variations $A_{\text {ellip }}$, Doppler beaming $A_{\text {beam }}$ (see also Loeb \& Gaudi 2003), reflected light eclipses and phase variations $A_{\text {ref }}$, and thermal light eclipses and phase variations $A_{\text {therm: }}$ :

$$
\begin{gathered}
A_{\text {beam }}=\alpha_{\text {beam }} 4\left(\frac{K}{c}\right) \sim 5.7 \alpha_{\text {beam }} \times 10^{-5} \\
A_{\text {ref }}=\alpha_{\text {ref }}\left(\frac{R_{P}}{a}\right)^{2} \sim 4.7 \alpha_{\text {ref }} \times 10^{-4} \\
A_{\text {ellip }}=\alpha_{\text {ellip }} \frac{M_{P}}{M_{*}}\left(\frac{R_{*}}{a}\right)^{3} \sim 4.1 \alpha_{\text {ellip }} \times 10^{-4} \\
A_{\text {therm }}=\alpha_{\text {therm }}\left(\frac{R_{P}}{R_{*}}\right)^{2}\left(\frac{R_{*}}{a}\right)^{1 / 2} \sim 3.2 \alpha_{\text {therm }} \times 10^{-3},
\end{gathered}
$$

where the expression for $A_{\text {therm }}$ assumes observations in the Rayleigh-Jeans tail of both objects, and the expression for $A_{\text {ellip }}$ assumes an edge-on orbit. The dimensionless constants $\alpha$ are defined in Mazeh \& Faigler (2010), but to make contact with the secondary eclipse analysis in Section 5.4 we note that $\alpha_{\text {ref }}=A_{g}$ and $\alpha_{\text {therm }}=\left[f^{\prime}\left(1-A_{B}\right)\right]^{1 / 4}$. All of these constants are expected to be of the order of unity, except for $\alpha_{\text {ref }}$, which may be quite low for strongly irradiated planets, depending on wavelength (Burrows et al. 2008). Based on previous results, all of these effects with the possible exception of Doppler beaming are likely to be detectable with precision photometry (see, e.g., Cowan et al. 2012). For ellipsoidal variations in particular, we expect $\alpha_{\text {ellip }} \sim 2$ and thus a relatively large amplitude of $A_{\text {ellip }} \sim 10^{-3}$. Furthermore, the detection of all these signals is facilitated by the short orbital period for KELT-1b.

The prospects for transmission spectroscopy are probably poorer, given the relatively small planet/star radius ratio $(\sim 0.078)$ and more importantly the large surface gravity for KELT-1b. For the optimistic case of $T_{\mathrm{eq}} \simeq 2400 \mathrm{~K}$ assuming zero albedo and perfect redistribution, the scale height is only $H \sim k T /\left(\mu \mathrm{m}_{H} g_{P}\right) \sim 16 \mathrm{~km}$, and thus will only lead to changes in the transit depth of order $\sim 2 H / R_{P} \sim 0.04 \%$.

\section{SUMMARY}

We have presented the discovery of KELT-1b, the first transiting low-mass companion from the wide-field KELTNorth transit survey. The host star KELT-1 is a mildly evolved, solar-metallicity, rapidly rotating, mid-F star with an age of $\sim 1.5-2$ Gyr located at a distance of $\sim 260 \mathrm{pc}$. The transiting companion is a low-mass brown dwarf or supermassive planet with mass $\sim 27 M_{\text {Jup }}$, on a very short period, circular orbit of $P \sim 1.2$ days.

In many ways, the KELT-1 system is quite unusual and extreme: KELT- 1 b receives a large amount of stellar insolation, is inflated relative to theoretical predictions, and raises strong tides on its host. The obliquity of KELT-1 is consistent with zero, and there is evidence that the spin of KELT-1 has been synchronized with the orbital period of KELT-1. Finally, there is a likely M-dwarf stellar companion to the KELT-1 system with a projected separation of $\sim 150$ AU. As the first definitively inflated transiting brown dwarf, KELT-1b demonstrates the need for models of brown dwarfs subject to a range of stellar insolations.

A plausible formation scenario for this system posits that KELT-1b formed on a much wider orbit and was driven to a smaller semimajor axis by the tertiary via the Kozai-Lidov mechanism. The system then continued to evolve under strong tidal forces, with KELT-1b migrating inward to its current orbit, while damping the obliquity of KELT-1 and synchronizing its spin period.

The future evolution of the KELT-1 system may be spectacular. As KELT-1 continues to evolve and its radius increases, so will the tides raised on it by KELT-1b. Assuming KELT-1 is and remains tidally locked, as it cools it will develop a deep convective envelope, but be forced to rotate at an ever increasing rate. In $\sim 2$ Gyr, KELT-1 will have roughly the temperature of Sun, but with a radius of $\sim 2 R_{\odot}$ and a rotational velocity of $\sim 100 \mathrm{~km} \mathrm{~s}^{-1}$. At this point, KELT-1 will likely become an active RS CVn star (Walter \& Bowyer 1981). Eventually, as KELT-1 reaches the base of the giant branch, it will swallow KELT-1b whole, likely resulting in a bright $\mathrm{UV} / \mathrm{X}$-ray and optical transient (Metzger et al. 2012).

We particularly thank Bruce Gary for acquiring and reducing the data from HAO, Saurav Dhital for estimating the chance probability of a close companion, and Bence Beky and the HATNet team for giving up one night on the FLWO $1.2 \mathrm{~m}$ on short notice. We thank Subo Dong, Jonathan Fortney, Fred Rasio, and Aristotle Socrates for useful discussions. We thank the referee, John Southworth, for a prompt and helpful report. Early work on KELT-North was supported by NASA Grant NNG04GO70G. Work by B.S.G., J.D.E., and T.G.B. was partially supported by NSF CAREER Grant AST-1056524. E.L.N.J. thanks Chris Capron and Peng Zhao for help with these observations, and gratefully acknowledges the support of the National Science Foundation's PREST program, which helped to establish the Peter van de Kamp Observatory through grant AST-0721386. K.A.C. was supported by a NASA Kentucky Space Grant Consortium Graduate Fellowship. J.A.P. and K.G.S. acknowledge support from the Vanderbilt Office of the Provost through the Vanderbilt Initiative in Data-intensive Astrophysics. K.G.S. and L.H. acknowledge the support of the National Science Foundation through PAARE grant AST-0849736 and AAG grant AST-1009810. The TRES and KeplerCam observations were obtained with partial support from the Kepler Mission through NASA Cooperative Agreement NNX11AB99A with the Smithsonian Astrophysical Observatory, PI: D.W.L.

This work has made use of NASA's Astrophysics Data System, the Exoplanet Orbit Database at exoplanets.org, and the Extrasolar Planet Encyclopedia at exoplanet.eu (Schneider et al. 2011).

\section{REFERENCES}

Agol, E., \& Steffen, J. 2006, MNRAS, 374, 941

Agol, E., Steffen, J., Sari, R., \& Clarkson, W. 2005, MNRAS, 359, 567

Alard, C. 2000, A\&AS, 144, 363

Alard, C., \& Lupton, R. H. 1998, ApJ, 503, 325

Alonso, R., Brown, T. M., Torres, G., et al. 2004, ApJ, 613, L153

Alsubai, K. A., Parley, N. R., Bramich, D. M., et al. 2011, MNRAS, 417, 709

Anderson, D. R., Collier Cameron, A., Gillon, M., et al. 2012, MNRAS, 422, 1988

Anderson, D. R., Collier Cameron, A., Hellier, C., et al. 2011, ApJ, 726, L19

Baglin, A. 2003, Adv. Space Res., 31, 345

Bakos, G. Á., Hartman, J., Torres, G., et al. 2011, ApJ, 742, 116

Bakos, G. Á., Hartman, J. D., Torres, G., et al. 2012, AJ, 144, 19

Bakos, G. Á., Noyes, R. W., Kovács, G., et al. 2007, ApJ, 656, 552 
Baraffe, I., Chabrier, G., Barman, T. S., Allard, F., \& Hauschildt, P. H. 2003, A\&A, 402, 701

Barnes, J. W. 2009, ApJ, 705, 683

Barnes, J. W., \& Fortney, J. J. 2004, ApJ, 616, 1193

Batalha, N. M., Rowe, J. F., Bryson, S. T., et al. 2012, arXiv:1202.5852

Beatty, T. G., Fernández, J. M., Latham, D. W., et al. 2007, ApJ, 663, 573

Beatty, T. G., Pepper, J., Siverd, R. J., et al. 2012, ApJ, 756, L39

Bensby, T., Feltzing, S., \& Lundström, I. 2003, A\&A, 410, 527

Borucki, W. J., Koch, D., Basri, G., et al. 2010, Science, 327, 977

Bertin, E., \& Arnouts, S. 1996, A\&AS, 117, 393

Bouchy, F., Bonomo, A. S., Santerne, A., et al. 2011a, A\&A, 533, A83

Bouchy, F., Deleuil, M., Guillot, T., et al. 2011b, A\&A, 525, A68

Brown, T. M., Charbonneau, D., Gilliland, R. L., Noyes, R. W., \& Burrows, A. 2001, ApJ, 552, 699

Buchhave, L. A., Bakos, G. Á., Hartman, J. D., et al. 2010, ApJ, 720, 1118

Burke, C. J., Gaudi, B. S., DePoy, D. L., \& Pogge, R. W. 2006, AJ, 132, 210

Burrows, A., Heng, K., \& Nampaisarn, T. 2011, ApJ, 736, 47

Burrows, A., Ibgui, L., \& Hubeny, I. 2008, ApJ, 682, 1277

Burrows, A., Marley, M., Hubbard, W. B., et al. 1997, ApJ, 491, 856

Butters, O. W., West, R. G., Anderson, D. R., et al. 2010, A\&A, 520, L10

Carter, J. A., \& Winn, J. N. 2009, ApJ, 704, 51

Carter, J. A., \& Winn, J. N. 2010, ApJ, 709, 1219

Carter, J. A., Winn, J. N., Holman, M. J., et al. 2011, ApJ, 730, 82

Charbonneau, D., Allen, L. E., Megeath, S. T., et al. 2005, ApJ, 626, 523

Charbonneau, D., Brown, T. M., Noyes, R. W., \& Gilliland, R. L. 2002, ApJ, 568,377

Claret, A., \& Bloemen, S. 2011, A\&A, 529, A75

Cody, A. M., \& Sasselov, D. D. 2002, ApJ, 569, 451

Collier Cameron, A., Bouchy, F., Hébrard, G., et al. 2007a, MNRAS, 375, 951

Collier Cameron, A., Guenther, E., Smalley, B., et al. 2010, MNRAS, 407, 507

Collier Cameron, A., Wilson, D. M., West, R. G., et al. 2007b, MNRAS, 380,1230

Coşkunoğlu, B., Ak, S., Bilir, S., et al. 2011, MNRAS, 412, 1237

Cowan, N. B., Machalek, P., Croll, B., et al. 2012, ApJ, 747, 82

Cumming, A., Butler, R. P., Marcy, G. W., et al. 2008, PASP, 120, 531

Cutri, R. M., Skrutskie, M. F., van Dyk, S., et al. 2003, VizieR Online Data Catalog, 2246, 0

Cutri, R. M., Skrutskie, M. F., van Dyk, S., et al. 2012, VizieR Online Data Catalog, 2311, 0

Darwin, G. H. 1879, Proc. R. Soc. Long., 29, 168

Deeg, H. J., Moutou, C., Erikson, A., et al. 2010, Nature, 464, 384

Deleuil, M., Deeg, H. J., Alonso, R., et al. 2008, A\&A, 491, 889

Demarque, P., Woo, J.-H., Kim, Y.-C., \& Yi, S. K. 2004, ApJS, 155, 667

Deming, D., Seager, S., Richardson, L. J., \& Harrington, J. 2005, Nature, 434, 740

Devor, J. 2005, ApJ, 628, 411

Dhital, S., West, A. A., Stassun, K. G., \& Bochanski, J. J. 2010, AJ, 139, 2566

Dodson-Robinson, S. E., Veras, D., Ford, E. B., \& Beichman, C. A. 2009, ApJ, 707,79

Doyle, L. R., Carter, J. A., Fabrycky, D. C., et al. 2011, Science, 333, 1602

Eastman, J., Gaudi, B. S., \& Agol, E. 2012, PASP, submitted (arXiv:1206.5798)

Eastman, J., Siverd, R., \& Gaudi, B. S. 2010, PASP, 122, 935

Fabrycky, D., \& Tremaine, S. 2007, ApJ, 669, 1298

Faigler, S., \& Mazeh, T. 2011, MNRAS, 415, 3921

Fleming, S. W., Ge, J., Barnes, R., et al. 2012, AJ, 144, 72

Ford, E. B., \& Gaudi, B. S. 2006, ApJ, 652, L137

Ford, E. B., \& Holman, M. J. 2007, ApJ, 664, L51

Fortney, J. J., Marley, M. S., \& Barnes, J. W. 2007, ApJ, 659, 1661

Fortney, J. J., \& Nettelmann, N. 2010, Space Sci. Rev., 152, 423

Fressin, F., Guillot, T., \& Nesta, L. 2009, A\&A, 504, 605

Fressin, F., Torres, G., Rowe, J. F., et al. 2012, Nature, 482, 195

Fúrész, G. 2008, PhD thesis, Univ. Szeged

Gaudi, B. S., Seager, S., \& Mallen-Ornelas, G. 2005, ApJ, 623, 472

Gaudi, B. S., \& Winn, J. N. 2006, ApJ, 655, 550

Ghez, A. M., Salim, S., Weinberg, N. N., et al. 2008, ApJ, 689, 1044

Gómez Maqueo Chew, Y., Stassun, K. G., Prša, A., \& Mathieu, R. D. 2009, ApJ, 699, 1196

Gould, A., \& Morgan, C. W. 2003, ApJ, 585, 1056

Guenther, D. B., Demarque, P., Kim, Y.-C., \& Pinsonneault, M. H. 1992, ApJ, 387,372

Guillot, T. 2005, Annu. Rev. Earth Planet. Sci., 33, 493

Grether, D., \& Lineweaver, C. H. 2006, ApJ, 640, 1051

Hansen, B. M. S. 2010, ApJ, 723, 285

Hansen, B. M. S., \& Barman, T. 2007, ApJ, 671, 861

Hartman, J. D., Bakos, G., Stanek, K. Z., \& Noyes, R. W. 2004, AJ, 128, 1761

Hartman, J. D., Gaudi, B. S., Holman, M. J., et al. 2008, ApJ, 675, 1254

Hartman, J. D., Gaudi, B. S., Holman, M. J., et al. 2009, ApJ, 695, 336
Hauschildt, P. H., Allard, F., \& Baron, E. 1999, ApJ, 512, 377

Hellier, C., Anderson, D. R., Collier Cameron, A., et al. 2009, Nature, 460, 1098 Hirano, T., Suto, Y., Winn, J. N., et al. 2011, ApJ, 742, 69

Høg, E., Fabricius, C., Makarov, V. V., et al. 2000, A\&A, 355, L27

Høg, E., Kuzmin, A., Bastian, U., et al. 1998, A\&A, 335, L65

Holman, M., Touma, J., \& Tremaine, S. 1997, Nature, 386, 254

Holman, M. J., Fabrycky, D. C., Ragozzine, D., et al. 2010, Science, 330, 51

Holman, M. J., \& Murray, N. W. 2005, Science, 307, 1288

Hut, P. 1980, A\&A, 92, 167

Hut, P. 1981, A\&A, 99, 126

Irwin, J., Buchhave, L., Berta, Z. K., et al. 2010, ApJ, 718, 1353

Jackson, B., Greenberg, R., \& Barnes, R. 2008, ApJ, 678, 1396

Johnson, D. R. H., \& Soderblom, D. R. 1987, AJ, 93, 864

Johnson, J. A., Apps, K., Gazak, J. Z., et al. 2011, ApJ, 730, 79

Johnson, J. A., Fischer, D. A., Marcy, G. W., et al. 2007, ApJ, 665, 785

Johns-Krull, C. M., McCullough, P. R., Burke, C. J., et al. 2008, ApJ, 677, 657

Kane, S. R., Mahadevan, S., von Braun, K., Laughlin, G., \& Ciardi, D. R. 2009, PASP, 121, 1386

Katz, B., Dong, S., \& Malhotra, R. 2011, Phys. Rev. Lett., 107, 181101

Kenyon, S. J., \& Hartmann, L. 1995, ApJS, 101, 117

Kipping, D. M. 2009, MNRAS, 392, 181

Kirkpatrick, J. D., Gelino, C. R., Cushing, M. C., et al. 2012, ApJ, 753, 156

Knutson, H. A., Charbonneau, D., Allen, L. E., Burrows, A., \& Megeath, S. T. 2008, ApJ, 673, 526

Kovács, G., Bakos, G., \& Noyes, R. W. 2005, MNRAS, 356, 557

Kovács, G., Zucker, S., \& Mazeh, T. 2002, A\&A, 391, 369

Kozai, Y. 1962, AJ, 67, 591

Kratter, K. M., Murray-Clay, R. A., \& Youdin, A. N. 2010, ApJ, 710, 1375

Kraus, A. L., \& Hillenbrand, L. A. 2007, AJ, 134, 2340

Kurucz, R. L. 1979, ApJS, 40, 1

Lang, D., Hogg, D. W., Mierle, K., Blanton, M., \& Roweis, S. 2010, AJ, 139,1782

Latham, D. W., Rowe, J. F., Quinn, S. N., et al. 2011, ApJ, 732, L24

Leconte, J., Chabrier, G., Baraffe, I., \& Levrard, B. 2010, A\&A, 516, A64

Lee, B. L., Ge, J., Fleming, S. W., et al. 2011, ApJ, 728, 32

Léger, A., Rouan, D., Schneider, J., et al. 2009, A\&A, 506, 287

Leggett, S. K., Golimowski, D. A., Fan, X., et al. 2002, ApJ, 564, 452

Lithwick, Y., \& Naoz, S. 2011, ApJ, 742, 94

Loeb, A., \& Gaudi, B. S. 2003, ApJ, 588, L117

Lidov, M. L. 1962, Planet. Space Sci., 9, 719

Lissauer, J. J., Fabrycky, D. C., Ford, E. B., et al. 2011, Nature, 470, 53

Lomb, N. R. 1976, Ap\&SS, 39, 447

Mandel, K., \& Agol, E. 2002, ApJ, 580, L171

Mandushev, G., Torres, G., Latham, D. W., et al. 2005, ApJ, 621, 1061

Marcy, G. W., \& Butler, R. P. 2000, PASP, 112, 137

Matsumura, S., Peale, S. J., \& Rasio, F. A. 2010, ApJ, 725, 1995

Mazeh, T., \& Faigler, S. 2010, A\&A, 521, L59

Mazeh, T., Nachmani, G., Sokol, G., Faigler, S., \& Zucker, S. 2012, A\&A, 541, A56

McCullough, P. R., Stys, J. E., Valenti, J. A., et al. 2006, ApJ, 648, 1228

McLaughlin, D. B. 1924, ApJ, 60, 22

Metzger, B. D., Giannios, D., \& Spiegel, D. S. 2012, MNRAS, 425, 2778

Mislis, D., \& Hodgkin, S. 2012, MNRAS, 422, 1512

Metchev, S. A., \& Hillenbrand, L. A. 2009, ApJS, 181, 62

Miller, N., \& Fortney, J. J. 2011, ApJ, 736, L29

Mohanty, S., \& Stassun, K. G. 2012, ApJ, 758, 12

Mohanty, S., Stassun, K. G., \& Doppmann, G. W. 2010, ApJ, 722, 1138

Mordasini, C., Alibert, Y., Benz, W., \& Naef, D. 2009, A\&A, 501, 1161

Muirhead, P. S., Johnson, J. A., Apps, K., et al. 2012, ApJ, 747, 144

Mullan, D. J., \& MacDonald, J. 2010, ApJ, 713, 1249

Naoz, S., Farr, W. M., Lithwick, Y., Rasio, F. A., \& Teyssandier, J. 2011, Nature, 473, 187

Nelder, J. A., \& Mead, R. 1965, Comput. J., 7, 308

O’Donovan, F. T., Charbonneau, D., Torres, G., et al. 2006, ApJ, 644, 1237

Ogilvie, G. I., \& Lin, D. N. C. 2004, ApJ, 610, 477

Ohta, Y., Taruya, A., \& Suto, Y. 2005, ApJ, 622, 1118

Pepper, J., Gould, A., \& Depoy, D. L. 2003, Acta Astron., 53, 213

Pepper, J., Pogge, R. W., DePoy, D. L., et al. 2007, PASP, 119, 923

Pont, F., Melo, C. H. F., Bouchy, F., et al. 2005, A\&A, 433, L21

Pont, F., Moutou, C., Bouchy, F., et al. 2006a, A\&A, 447, 1035

Pont, F., Zucker, S., \& Queloz, D. 2006b, MNRAS, 373, 231

Quinn, S. N., Bakos, G. Á., Hartman, J., et al. 2012, ApJ, 745, 80

Rafikov, R. R. 2005, ApJ, 621, L69

Rasio, F. A., Tout, C. A., Lubow, S. H., \& Livio, M. 1996, ApJ, 470, 1187

Rogers, L. A., \& Seager, S. 2010, ApJ, 712, 974

Rossiter, R. A. 1924, ApJ, 60, 15

Rowe, J. F., Borucki, W. J., Koch, D., et al. 2010, ApJ, 713, L150 
Sahlmann, J., Ségransan, D., Queloz, D., et al. 2011, A\&A, 525, A95

Scargle, J. D. 1982, ApJ, 263, 835

Schneider, J., Dedieu, C., Le Sidaner, P., Savalle, R., \& Zolotukhin, I. 2011, A\&A, 532, A79

Schwarzenberg-Czerny, A. 1989, MNRAS, 241, 153

Seager, S. 2010, Exoplanet Atmospheres: Physical Processes (Princeton: Princeton Univ. Press)

Seager, S., \& Deming, D. 2010, ARA\&A, 48, 631

Seager, S., \& Hui, L. 2002, ApJ, 574, 1004

Seager, S., \& Mallén-Ornelas, G. 2003, ApJ, 585, 1038

Seager, S., \& Sasselov, D. D. 2000, ApJ, 537, 916

Skrutskie, M. F., Cutri, R. M., Stiening, R., et al. 2006, AJ, 131, 1163

Socrates, A., Katz, B., Dong, S., \& Tremaine, S. 2012, ApJ, 750, 106

Southworth, J. 2009, MNRAS, 394, 272

Spiegel, D. S., Burrows, A., \& Milsom, J. A. 2011, ApJ, 727, 57

Spiegel, D. S., Haiman, Z., \& Gaudi, B. S. 2007, ApJ, 669, 1324

Stassun, K. G., Mathieu, R. D., \& Valenti, J. A. 2007, ApJ, 664, 1154

Steffen, J. H., \& Agol, E. 2005, MNRAS, 364, L96

Steffen, J. H., Batalha, N. M., Borucki, W. J., et al. 2010, ApJ, 725, 1226

Steffen, J. H., Ragozzine, D., Fabrycky, D. C., et al. 2012, Proc. Natl Acad. Sci., 109,7982

Stetson, P. B. 1987, PASP, 99, 191

Stetson, P. B. 1990, PASP, 102, 932 ter Braak, C. J. F. 2006, Stat. Comput., 16, 239

Torres, G., Andersen, J., \& Giménez, A. 2010, A\&AR, 18, 67

Tusnski, L. R. M., \& Valio, A. 2011, ApJ, 743, 97

van Saders, J. L., \& Pinsonneault, M. H. 2012, ApJ, 746, 16

Vidal-Madjar, A., Lecavelier des Etangs, A., Désert, J.-M., et al. 2003, Nature, 422, 143

Walter, F. M., \& Bowyer, S. 1981, ApJ, 245, 671

Welsh, W. F., Orosz, J. A., Carter, J. A., et al. 2012, Nature, 481, 475

Winn, J. N. 2009, in IAU Symp. 253, Transiting Planets (Cambridge: Cambridge Univ. Press), 99

Winn, J. N. 2010, in Exoplanets, ed. S. Seager (Tucson, AZ: Univ. of Arizona Press), 55

Winn, J. N., Fabrycky, D., Albrecht, S., \& Johnson, J. A. 2010, ApJ, 718, L145

Winn, J. N., Holman, M. J., Torres, G., et al. 2008, ApJ, 683, 1076

Winn, J. N., Noyes, R. W., Holman, M. J., et al. 2005, ApJ, 631, 1215

Wright, E. L., Eisenhardt, P. R. M., Mainzer, A. K., et al. 2010, AJ, 140, 1868

Wright, J. T., Fakhouri, O., Marcy, G. W., et al. 2011, PASP, 123, 412

Wright, J. T., Marcy, G. W., Howard, A. W., et al. 2012, ApJ, 753, 160

Wu, Y., Murray, N. W., \& Ramsahai, J. M. 2007, ApJ, 670, 820

Zacharias, N., Monet, D. G., Levine, S. E., et al. 2004, BAAS, 36, 1418

Zucker, S., Mazeh, T., Santos, N. C., Udry, S., \& Mayor, M. 2003, A\&A, 404, 775 